\title{
Minimal Taylor Algebras as a Common Framework for the Three Algebraic Approaches to the CSP
}

\author{
* Libor Barto ${ }^{1}$, Zarathustra Brady², Andrei Bulatov ${ }^{3}$, Marcin Kozik ${ }^{4}$, and Dmitriy \\ Zhuk $^{5}$ \\ ${ }^{1}$ Faculty of Mathematics and Physics, Charles University, Prague, Czechia, email: \\ libor.barto@gmail.com \\ ${ }^{2}$ Email: notzeb@gmail.com \\ ${ }^{3}$ School of Computing Science, Simon Fraser University, Burnaby BC, Canada, \\ email: abulatov@sfu.ca \\ ${ }^{4}$ Theoretical Computer Science Department, Faculty of Mathematics and \\ Computer Science, Jagiellonian University, Krakow, Poland, \\ email:marcin.kozik@uj.edu.pl \\ ${ }^{5}$ HSE University, Russia and Lomonosov Moscow State University, Russia, email: \\ zhuk@intsys.msu.ru
}

April 27, 2021

\begin{abstract}
This paper focuses on the algebraic theory underlying the study of the complexity and the algorithms for the Constraint Satisfaction Problem (CSP). We unify, simplify, and extend parts of the three approaches that have been developed to study the CSP over finite templates - absorption theory that was used to characterize CSPs solvable by local consistency methods (JACM'14), and Bulatov's and Zhuk's theories that were used for two independent proofs of the CSP Dichotomy Theorem (FOCS'17, JACM'20).

As the first contribution we present an elementary theorem about primitive positive definability and use it to obtain the starting points of Bulatov's and Zhuk's proofs as corollaries. As the second contribution we propose and initiate a systematic study of minimal Taylor algebras. This class of algebras is broad enough so that it suffices to verify the CSP Dichotomy Theorem on this class only, but still is unusually well behaved. In particular, many concepts from the three approaches coincide in the class, which is in striking contrast with the general setting.

We believe that the theory initiated in this paper will eventually result in a simple and more natural proof of the Dichotomy Theorem that employs a simpler and more efficient algorithm, and will help in attacking complexity questions in other CSP-related problems.
\end{abstract}

\section{Introduction}

The Constraint Satisfaction Problem (CSP) has attracted much attention from researchers in various disciplines. One direction of the CSP research has been greatly motivated by the so-called

${ }^{*}$ Libor Barto has received funding from the European Research Council (ERC) under the European Unions Horizon 2020 research and innovation programme (grant agreement No 771005). Zarathustra Brady has received funding from the NSF Mathematical Sciences Postdoctoral Research Fellowship under Grant No. (DMS-1705177). Andrei Bulatov has received funding from an NSERC Discovery grant. Marcin Kozik was partially supported by National Science Centre, Poland projects 2014/2013/B/ST6/01812 and 2014/14/A/ST6/00138. Dmitriy Zhuk was supported by HSE University Basic Research Program. 
Dichotomy Conjecture of Feder and Vardi [34, 35] that concerns the computational complexity of CSPs over finite relational structures. The Constraint Satisfaction Problem over a finite relational structure $\mathbb{A}$ of finite signature (also called a template), in its logical formulation, is the problem to decide the validity of a given primitive positive sentence ( $p p$-sentence), i.e., a sentence that is an existentially quantified conjunction of atomic formulas over $\mathbb{A}$ - the constraints. Examples of problems in this class include satisfiability problems, graph coloring problems, and solving systems of equations over finite algebraic structures (see [38, 41, 8, 48]), the CSP is also ubiquitous in artificial intelligence 33 .

A classic result in the field is a theorem by Schaefer [53] that completely classifies the complexity of CSPs over relational structures with a two-element domain, so-called Boolean structures, by providing a dichotomy theorem: each such a CSP is either solvable in polynomial time or is NP-complete. The Dichotomy Conjecture of Feder and Vardi then states that Schaefer's result extends to arbitrary finite domains. This conjecture inspired a very active research program in the last 20 years, culminating in a positive resolution independently obtained by Bulatov 24 and Zhuk [54, 55]. The exact borderline between tractability and hardness can be formulated as follows [27, 9, 8].

Theorem 1.1. Let $\mathbb{A}$ be a finite relational structure over a finite signature.

- If every finite structure is homomorphically equivalent to a finite structure pp-interpretable in $\mathbb{A}$, then the CSP over $\mathbb{A}$ is NP-hard,

- otherwise it is solvable in polynomial time.

It was already recognized in Schaefer's work (in fact, it was the basis of his approach) that the complexity of a CSP depends only on the set of relations that are pp-definable (i.e., definable by a primitive positive formula) from the template. Such sets of relations are now usually referred to as relational clones. The impetus of rapid development in the area after Feder and Vardi's seminal work [35] was a series of papers [41, 42] that brought to attention and applied a Galois connection between operations and relations studied in the sixties [37, 13, which gives a bijective correspondence between relational clones and clones - sets of term operations of algebras.

One way to phrase this core fact is as follows: for any finite algebra $\mathbf{A}$, its set of invariant relations (subuniverses of powers or subpowers in algebraic terminology) is always a relational clone; every relational clone is of this form; and two algebras have the same relational clone of subpowers if and only if they have the same set of term operations. For instance, a Boolean CSP, say over the domain $\{0,1\}$, is solvable in polynomial time if and only if the relations of the template are subpowers of one of four types of algebras - an algebra with a single constant operation, a semilattice, the majority algebra, or the affine Mal'cev algebra of $\mathbb{Z} / 2$ (see Subsection 3.1).

This connection between relations and operations allowed researchers to apply techniques from Universal Algebra. Application of these techniques became known as the algebraic approach to the $C S P$, although one may argue that the term misses the point a little - the success of the approach lies mostly in combining and moving back and forth between the relational and algebraic side, and this is the case for this paper as well. The general theory of the CSP was further refined in subsequent papers [27, 9] and turned out to be an efficient tool in other types of constraint problems including the Quantified CSP [14, 31, 57, the Counting CSP [26, 21, some optimization problems, e.g. the Valued CSP [47] and robust approximability [5], infinite-domain CSPs [12, and related promise problems such as "approximate coloring" and the Promise CSP [16, 30], and many others.

One useful technical finding of [27] is that every CSP is equivalent to a CSP over an idempotent template, i.e. a template that contains all the singleton unary relations. This allows us to use parameters in pp-definitions and omit homomorphic equivalence in the first item of Theorem 1.1. On the algebraic side, this allows us to concentrate on so-called idempotent algebras (see Subsection 2.1). Another important contribution of that paper was a conjecture postulating, for idempotent structures, the exact borderline between polynomial solvability and NP-hardness, which coincides with the borderline stated in Theorem 1.1. The hardness part was already dealt 
with in the same paper and what was left was the tractability part. Within the realm of idempotent structures, the algebras corresponding to the second item of Theorem 1.1 are so-called Taylor algebras (see Subsection 3.1). The following theorem is therefore the core of the two proofs of the Dichotomy Conjecture.

Theorem 1.2 (24, 54, 55]). Let $\mathbb{A}$ be an idempotent structure. If there exists an idempotent Taylor algebra $\mathbf{A}$ such that all relations in $\mathbb{A}$ are subpowers of $\mathbf{A}$, then the CSP over $\mathbb{A}$ is solvable in polynomial time.

Partial results toward Theorem 1.2 include dichotomies for various classes of relational structures and algebras (e.g. the class of 3-element algebras [19] and the class of structures containing all unary relations [20), understanding of the limits of algorithmic techniques (e.g. local consistency methods 4 and describing generating sets of solutions [40]), and finding potentially useful characterizations of Taylor algebras (e.g. by means of weak near-unanimity operations [50] and by means of cyclic operations [3]). The papers [4] and [3] initiated a technique, now referred to as the absorption theory [6]. It is one of the fruits of CSP-motivated research that impacted also other CSP-related problems as well as universal algebra (e.g. [1]) and it is one of the three theories this paper is concerned with.

Bulatov and Zhuk in their resolution of the Dichotomy Conjecture (and their prior and subsequent work) developed novel techniques, which we refer to as Bulatov's theory and Zhuk's theory in this paper. These theories are (understandably) mostly focused on the task at hand, to prove Theorem 1.2, and as such have several shortcomings. First, some of the new concepts are still evolving as the need arises and they do not yet feel quite elegant and settled. Moreover, the theories are technically complex which makes it difficult to master them and to apply them in different contexts. This is best witnessed by the absence of results from different authors that would employ the theories (needless to say they have already clearly witnessed their potential). Second, they both employ the following trick. Instead of studying a general, possibly wild Taylor algebra, one can first tame it by taking a certain Taylor reduct - an algebra whose operations are only some of the term operations but which is still Taylor. Taking reducts does not result in any loss of generality in Theorem 1.2, since reducts keep all the original invariant relations, so proving tractability for a reduct is sufficient for tractability for the original problem. However, taking reducts does result in loss of generality of the theory and it is not yet clear to which natural classes of algebras the theories apply. Moreover, these reducts are different in the two approaches. Third, connections between Bulatov's and Zhuk's theories were not understood at all. While Zhuk's theory and absorption theory at least had some concepts in common, Bulatov's theory seemed quite orthogonal to the rest.

The contributions of this paper unify, simplify, and extend parts of these three theories, making them, we hope, more accessible and reducing the prerequisites for the dichotomy proofs. In particular, we initiate a systematic study of minimal Taylor algebras, i.e., those algebras that are Taylor but such that none of their proper reducts is Taylor. Thus, we employ the above trick to the extreme and study, in a sense, the tamest algebras or, in other words, "hardest" tractable CSPs. This restriction, on the one hand, limits the scope of the theory but, on the other hand, gives us a framework in which the three theories do not look separate at all anymore, as we shall see.

Even though our unifications, simplifications, and extensions do not cover some advanced parts of the three theories (more on this in due course and Section 17), we believe that they have the potential to evolve into one coherent theory of finite algebras that would make the CSP Dichotomy Theorem an exercise (albeit hard) and that would have applications well beyond constraint problems.

The contributions can be divided into two groups, results for (all finite) Taylor algebras stated in Section 4 and results for minimal Taylor algebras in Sections 5 and 6. We now describe them in more detail together with more background. 


\subsection{Taylor algebras}

The central concept in absorption theory is that of absorbing subuniverses introduced formally in Subsection 3.3. These are invariant subsets of algebras with an additional property resembling ideals in rings. A fundamental theorem, the absorption theorem, shows that nontrivial absorbing subuniverses in Taylor algebras exist under rather mild conditions and this fact makes the theory applicable in many situations. For instance, the strategy in [4 to provide a global solution to a locally consistent instance is to propagate local consistency into proper absorbing subuniverses. The abundance of absorption provided by absorption theorem makes this propagation often possible, and if it is not, gives us sufficient structural and algebraic information about the instance which makes the propagation possible nevertheless, until the instance becomes trivially solvable.

Zhuk's starting point is a theorem stating that every Taylor algebra has a proper subuniverse of one of four special types (see Subsections 3.3 and 4.3). Zhuk derives the four types theorem from a complicated result in clone theory, Rosenberg's classification of maximal clones [52] (the dependence of this approach on Rosenberg's result is removed in [56]). Given the four types theorem, the overall strategy for the polynomial algorithm for Theorem 1.2 is natural and similar in spirit to the absorption technique - to keep reducing to one of such subuniverses until the problem becomes trivial. Although Zhuk's theory has a nontrivial intersection with the absorption theory, these connections were not properly explored and verbalized.

Bulatov's algorithm in his proof of Theorem 1.2 employs a similar general idea, he reduces the instance to certain subuniverses. However, these special subuniverses are defined, as opposed to absorption and Zhuk's theories, in a very local way. They are sets that are, in a sense, closed under edges (e.g. strong components) of a labeled directed graph whose vertices are the elements of the algebra. Bulatov introduces three basic kinds of edges (see Subsection [3.4), whose presence indicates that the local structure around the adjacent vertices, namely the subuniverse generated by the two vertices, somewhat resembles the three interesting tractable cases in Schaefer's Boolean dichotomy. What makes this approach work is a fundamental theorem (Theorem 1 [22], see also [18]), which says that the edges sufficiently approximate the algebra in the sense that the directed graph is connected. The proof uses rather technically challenging constructions involving operations in the algebra.

In Section 4 we first describe some of the connections between absorption theory and Zhuk's theory, and explain simplifications and refinements that were scattered across literature, including a refinement of the absorption theorem that follows from [54, 55]. We also give two new results improving pieces of the two theories. The major novel contribution of Section 4 is Theorem 4.7 a purely relational fact which roughly states that each "interesting" relation that uses all the domain elements in every coordinate pp-defines a binary relation with the same properties or a ternary relation of a very particular shape. Although the proof is elementary and not very long, it enables us to derive both Zhuk's four types theorem and Bulatov's connectivity theorems as corollaries. It may be also of interest for some readers to note that theorems in this section often even do not require the algebra to be Taylor - they concern all finite idempotent algebras.

\subsection{Minimal Taylor algebras}

The advantage of studying minimal reducts within a class of interest was clearly demonstrated in the work of Brady [15. He concentrated on so-called bounded width algebras - these are algebras that play the same role in solvability of CSPs by local consistency methods 4 as Taylor algebras do for polynomial time solvability. The theory he developed enabled him to classify all the minimal bounded width algebras on small domains. Our first contributions in Section 5 show that the basic facts for minimal bounded width algebras have their counterparts for minimal Taylor algebras. For instance, Proposition 5.2 shows that every Taylor algebra does have a minimal Taylor reduct, and so minimal Taylor algebras are indeed sufficiently general, e.g., in the CSP context.

The authors find the extent, to which the notions of the three theories simplify and unify in minimal Taylor algebras, truly striking. Our major results in this direction are Theorems 5.7, 5.9, 5.12, 5.18, 5.20, 5.22, 5.23 in Section 5 and their consequences stated in Section 6, where various 
classes of algebras are characterized in terms of types of edges, types of operations, and types of absorption present in the algebras. We now discuss a sample of the obtained results.

Edges, as we already mentioned, are pairs of elements for which the local structure around the pair resembles one of the three interesting polynomially solvable cases in Schaefer's Boolean dichotomy [53. More precisely, and specializing to one kind of edges, we say that $(a, b)$ is a majority edge if the subalgebra $\mathbf{E}$ generated by $a$ and $b$ has a proper congruence (i.e., invariant equivalence relation) $\theta$ and a term operation $t$ that acts as the majority operation on the blocks $a / \theta$ and $b / \theta$. The resemblance of the two-element majority algebra is in general quite loose - the equivalence $\theta$ can have many more blocks and there may be many more operations in $\mathbf{E}$ other than $t$. However, in minimal Taylor algebras, $\mathbf{E}$ modulo $\theta$ is always term equivalent to the two element majority algebra.

The second sample concerns the simplest absorbing subuniverses, the 2-absorbing ones, which constitute one of the four types of Zhuk's fundamental theorem. The 2-absorption of a subuniverse $B$ is a relatively strong property that requires the existence of some binary term operation $t$ whose result is always in $B$ provided at least one of the arguments is in $B$. An extreme further strengthening is as follows: the result of applying any operation $f$ to an argument that contains an element in $B$ in any essential coordinate is in $B$. It turns out that these notions actually coincide for minimal Taylor algebras. What is perhaps even more surprising is the connection to Bulatov's theory: 2-absorbing sets are exactly subsets stable (in a certain sense) under all the three kinds of edges.

Finally, we mention that the clone of any minimal Taylor algebra is generated by a single ternary operation. This, together with other structural results in this paper, may help in enumerating Taylor algebras - at the very least we know that there are at most $n^{n^{3}}$ of them over a domain of size $n$. Such a catalogue could be a valuable source of examples for CSP-related problems as well as universal algebra. Additionally, having a complete catalogue of minimal Taylor algebras for a given domain allows you to write down an explicit, concrete generalization of Schaefer's Dichotomy Theorem [53] for a domain of that size, with as few cases as possible.

Brady has already initiated this project and has found all the three-element minimal Taylor algebras in unpublished work based on the results of this paper. Up to term-equivalence and permutations of the domain, there are exactly 24 minimal Taylor algebras on a domain of size 3 . This gives us a concrete list of the hardest tractable CSPs on the 3-element domain, refining the main result of [19].

\section{Preliminaries}

\subsection{Algebras}

Algebras, i.e. structures with purely functional signature, will be denoted by boldface capital letters (e.g., A) and their universes (also called domains) typically by the same letter in the plain font (e.g., A). The basic general algebraic concepts, such as subuniverses, subalgebras, products, and quotients modulo congruences are used in the standard way (see, e.g. [10]). An algebra is nontrivial if it has more than two elements, otherwise it is trivial. We use $B \leq \mathbf{A}$ to mean that $B$ is a subuniverse of $\mathbf{A}$. By a subpower we mean a subuniverse (or a subalgebra) of a finite power. Recall that subpowers are the same as invariant relations and we may also call them compatible relations. The set of all subpowers is denoted $\operatorname{Inv}(\mathbf{A})$. The subuniverse (or the subalgebra) of $\mathbf{A}$ generated by a set $X \subseteq A$ is denoted $\operatorname{Sg}_{\mathbf{A}}(X)$ or $\operatorname{Sg}_{\mathbf{A}}\left(x_{1}, \ldots, x_{n}\right)$ when $X=\left\{x_{1}, \ldots, x_{n}\right\}$.

All theorems in this paper concern algebras that are finite and idempotent, that is, $f(x, x, \ldots, x)=x$ for every operation $f$ in the algebra and every element $x$ of the universe. Recall that this is not a severe restriction, at least in the area of finite-template CSPs. We do not explicitly mention this assumption in the statements of theorems or definitions.

A (function) clone is a set of operations $\mathscr{C}$ on a set $A$ which contains all the projections $\operatorname{proj}_{i}^{n}$ (the $n$-ary projection to the $i$-th coordinate) and is closed under composition, i.e., $f\left(g_{1}, \ldots, g_{n}\right) \in \mathscr{C}$ whenever $f \in \mathscr{C}$ is $n$-ary and $g_{1}, \ldots, g_{n} \in \mathscr{C}$ are all $m$-ary, where $f\left(g_{1}, \ldots, g_{n}\right)$ denotes the 
operation defined by $f\left(g_{1}\left(x_{1}, \ldots, x_{m}\right), \ldots, g_{n}\left(x_{1}, \ldots, x_{m}\right)\right)$. By $\mathrm{Clo}(\mathbf{A})\left(\operatorname{Clo}_{n}(\mathbf{A})\right.$, respectively), we denote the clone of all term operations (the set of all $n$-ary term operations, respectively) of A. An algebra $\mathbf{B}$ is a reduct of $\mathbf{A}$ if they have the same universe $A=B$ and $\mathrm{Clo}(\mathbf{B}) \subseteq \operatorname{Clo}(\mathbf{A})$. Algebras $\mathbf{A}$ and $\mathbf{B}$ are term-equivalent if each of them is a reduct of the other, i.e., $\operatorname{Clo}(\mathbf{A})=$ $\mathrm{Clo}(\mathbf{B})$.

A coordinate $i$ of an operation $f: A^{n} \rightarrow A$ is essential if $f$ depends on the $i$ th coordinate, i.e., $f(\mathbf{a}) \neq f(\mathbf{b})$ of some tuples $\mathbf{a}, \mathbf{b} \in A^{n}$ that differ only at the $i$ th coordinate.

\subsection{Relations}

A relation on $A$ is a subset of $A^{n}$, but we often work with more general "multisorted" relations $R \subseteq A_{1} \times A_{2} \times \cdots \times A_{n}$. We call such an $R$ proper if $R \neq A_{1} \times \cdots \times A_{n}$ and nontrivial if it is nonempty and proper. Tuples are written in boldface and components of $\mathbf{x} \in A_{1} \times \cdots \times A_{n}$ are denoted $x_{1}, x_{2}, \ldots$ Both $\mathbf{x} \in R$ and $R(\mathbf{x})$ are used to denote the fact that $\mathbf{x}$ is in $R$. The projection of $R$ onto the coordinates $i_{1}, \ldots, i_{k}$ is denoted $\operatorname{proj}_{i_{1}, \ldots, i_{k}}(R)$. The relation $R$ is subdirect, denoted $R \subseteq s d A_{1} \times \cdots \times A_{n}$, if $\operatorname{proj}_{i}(R)=A_{i}$ for each $i$. We call $R$ redundant, if there exist coordinates $i \neq j$ such that $\operatorname{proj}_{i j}(R)$ is a graph of bijection from $A_{i}$ to $A_{j}$; otherwise $R$ is irredundant.

We say that a set of relations $\mathcal{R}$ pp-defines $S$ if $S$ can be defined from $\mathcal{R}$ by a primitive positive formula with parameters, that is, using the existential quantifier, relations from $\mathcal{R}$, the equality relation, and the singleton unary relations. Recall that the set of subpowers of an algebra is closed under pp-definitions.

For binary relations we write $-R$ instead of $R^{-1}$ and $R+S$ for the relational composition of $R$ and $S$, that is $R+S=\{(a, c):(\exists b) R(a, b) \wedge R(b, c)\}$. For a unary relation $B$ we write $B+S$ to denote the set $\{c:(\exists b) B(b) \wedge S(b, c)\}$ and if $B$ is a singleton we often write $b+S$ instead of $\{b\}+S$. Also, we set $R-S=R+(-S)=R \circ S^{-1}$. A relation $R \subseteq A \times B$ is linked if $(R-R)+(R-R)+\cdots+(R-R)$ is equal to $\left(\operatorname{proj}_{1}(R)\right)^{2}$ for some number of summands. In other words, $R$ is connected when viewed as a bipartite graph between $A$ and $B$ (with possible isolated vertices). The left center of $R \subseteq A \times B$ is the set $\{a \in A: a+R=B\}$. If $R$ has a nonempty left center, it is called left central. Right center and right central relations are defined analogically. A relation is central if it is left central and right central. Note that $R+S,-R$, and the left (right) center of $R$ are pp-definable from $\{R, S\}$.

\section{Basic concepts}

\subsection{Taylor algebras}

First we define the central concept of the algebraic theory of the CSP, Taylor algebra. From the many equivalent definitions (e.g., the one using so-called Taylor operations - that's where the name comes from) we present a direct algebraic counterpart of the first item in Theorem 1.1 specialized to idempotent structures.

Definition 3.1. An (idempotent, finite) algebra $\mathbf{A}$ is a Taylor algebra if no quotient of a subpower of $\mathbf{A}$ is a two-element algebra whose every operation is a projection.

We will often take advantage of the characterization by means of cyclic operation. Its original proof is via the absorption theory, an alternative proof is now available using Zhuk's approach [56].

Theorem 3.2 ([3]). The following are equivalent for any algebra.

- A is Taylor.

- There exists $n>1$ such that $\mathbf{A}$ has a term operation $t$ of arity $n$ which is cyclic, that is, for any $\mathbf{x} \in A^{n}$,

$$
t\left(x_{1}, x_{2}, \ldots, x_{n}\right)=t\left(x_{2}, \ldots, x_{n}, x_{1}\right)
$$

- For every prime $p>|A|$, A has a term operation $t$ of arity $p$ which is cyclic. 
Several further types of operations are significant for this paper:

- Semilattice operation is a binary operation $\vee$ which is commutative, idempotent, and associative.

- Majority operation is a ternary operation $m$ satisfying $m(x, x, y)=m(x, y, x)=m(y, x, x)=$ $x$ (for any $x, y$ in the universe).

- Mal'cev operation is a ternary operation $p$ satisfying $p(y, x, x)=p(x, x, y)=y$.

Any algebra with a semilattice, or majority, or Mal'cev operation is Taylor. The following algebras are particularly important for our purposes (recall also the discussion about Schaefer's result in the introduction):

- Two-element semillatice: a two-element set together with one of the two semilattice operations, e.g., $(\{0,1\} ; \vee)$ where $\vee$ is the maximum operation,

- Two-element majority algebra: a two element set together with the unique majority operation, e.g., $\left(\{0,1\} ;\right.$ maj). We also use $\operatorname{maj}_{p}$, for odd $p$, to denote the $p$-ary majority operation on $\{0,1\}$, that is, $\operatorname{maj}(\mathbf{a})=1$ iff the majority of the $a_{i}$ is 1 .

- Affine Mal'cev algebra: a set together with the Mal'cev operation $x-y+z$, where + and - is computed with respect to a fixed abelian group structure on the universe, e.g., $(\{0,1, \ldots, p-1\} ; x-y+z(\bmod p))$. More generally, an affine module is an algebra whose term operations are exactly the idempotent term operations of a module over a unital ring.

\subsection{Abelian algebras}

The last example falls into a larger class of algebras, which is also significant in the algebraic theory of CSPs and Universal Algebra in general, so-called abelian algebras.

Definition 3.3. An algebra $\mathbf{A}$ is abelian if the diagonal $\Delta_{A}=\{(a, a): a \in A\}$ is a block of a congruence of $\mathbf{A}^{2}$.

As an example, for an affine Mal'cev algebra, a congruence satisfying the definition is the congruence $\alpha$ defined by

$$
\left(\left(x_{1}, x_{2}\right),\left(y_{1}, y_{2}\right)\right) \in \alpha \text { iff } x_{1}-x_{2}=y_{1}-y_{2} .
$$

Note that an abelian algebra does not need to be Taylor, e.g., an algebra with no operations is such (except in the pathological, one-element case). However, for (finite, idempotent) Taylor algebras, abelian algebras admit a complete description up to term equivalence. The original proof of this result is using the tame congruence theory (a developed theory of finite algebras that we have not mentioned yet), an alternative proof using absorption is available [3].

Theorem 3.4 (39]). Every (finite, idempotent) abelian Taylor algebra is an affine module.

In light of this theorem, readers interested solely in Taylor algebras may safely replace the phrase "abelian algebra" with "affine module". In fact, it follows from our results that in minimal Taylor algebras "affine module" can be further simplified to "affine Mal'cev algebra", up to term equivalence.

\subsection{Absorption}

Now we introduce absorbing subuniverses, centers, and projective subuniverses, central concepts in the absorption theory and Zhuk's theory.

Definition 3.5. Let $\mathbf{A}$ be an algebra and $B \subseteq A$. We call $B$ an n-absorbing set of $\mathbf{A}$ if there is a term operation $t \in \mathrm{Clo}_{n}(\mathbf{A})$ such that $t(\mathbf{a}) \in B$ whenever $\mathbf{a} \in A^{n}$ and $\left|\left\{i: a_{i} \in B\right\}\right| \geq n-1$.

If, additionally, $B$ is a subuniverse of $\mathbf{A}$, we write $B \unlhd_{n} \mathbf{A}$, or $B \unlhd \mathbf{A}$ when the arity is not important. 
We also say " $B$ absorbs $\mathbf{A}$ (by $t$ )" in the situation of Definition 3.5. Of particular interest for us are $n$-absorbing subuniverse with $n=2$, e.g., $\{1\}$ in the semilattice $(\{0,1\} ; \vee))$, or $n=3$, e.g., $\{0\}$ and $\{1\}$ in the two-element majority algebra.

The concept of a center is still evolving and it is not yet clear what the best version would be for general algebras. Our definition follows [55], although a more recent paper [56] made an adjustment motivated by this work. As we shall see in Theorem 5.9 the situation is much cleaner for minimal Taylor algebras.

Definition 3.6. $A$ subset $B \subseteq A$ is a center of $\mathbf{A}$ if there exists an algebra $\mathbf{C}$ (of the same signature) with no nontrivial 2-absorbing subuniverse and $R \leq_{\text {sd }} \mathbf{A} \times \mathbf{C}$ such that $B$ is the left center of $R$. The relation $R$ is called a witnessing relation. If $\mathbf{C}$ can be chosen Taylor, we call $B$ a Taylor center of $\mathbf{A}$.

The final concept that we introduce in this section is a projective subuniverse. It appeared in [49] in connection with so-called cube operations, which characterize the limit of the few subpowers algorithm that finds generating set of all solutions to a CSP [40]. These subuniverses were called cube term blockers in [49] but it became clear that the concept is significant beyond this context [7, 56] and for this reason we prefer the terminology from the latter paper.

Definition 3.7. Let $\mathbf{A}$ be an algebra and $B \subseteq A$. We say that $B$ is a projective subuniverse if for every $f \in \mathrm{Clo}_{n}(\mathbf{A})$ there exists a coordinate $i$ of $f$ such that $f(\mathbf{a}) \in B$ whenever $\mathbf{a} \in A^{n}$ is such that $a_{i} \in B$.

Note that a projective subuniverse of $\mathbf{A}$ is, indeed, a subuniverse. Also note that centers are automatically subuniverses as well.

Many of the algebraic concepts that we introduce (such as absorbing subuniverses or strongly projective subuniverses from Section [5) have a useful equivalent characterizations in terms of relations. Such a characterization for projective subuniverses is especially elegant and we state it here for reference.

Proposition 3.8 (Lemma 3.2 in [49]). Let $\mathbf{A}$ be an algebra and $B \subseteq A$. Then $B$ is a projective subuniverse of $\mathbf{A}$ if and only if, for every $n$, the relation $B\left(x_{1}\right) \vee B\left(x_{2}\right) \vee \cdots \vee B\left(x_{n}\right)$ is a subpower of $\mathbf{A}$.

\subsection{Edges}

Finally we introduce the three types of edges used in Bulatov's approach to the CSP.

Definition 3.9. Let $\mathbf{A}$ be an algebra. A pair $(a, b) \in A^{2}$ is an edge if there exists a proper congruence $\theta$ on $\operatorname{Sg}_{\mathbf{A}}(a, b)$ ( $a$ witness for the edge) such that one of the following happens:

- (semilattice edge) There is a term operation $f \in \mathrm{Clo}_{2}(\mathbf{A})$ acting as a join semilattice operation on $\{a / \theta, b / \theta\}$ with top element $b / \theta$.

- (majority edge) There is a term operation $m \in \mathrm{Clo}_{3}(\mathbf{A})$ acting as a majority operation on $\{a / \theta, b / \theta\}$.

- (abelian edge) The algebra $\operatorname{Sg}_{\mathbf{A}}(a, b) / \theta$ is abelian.

An edge $(a, b)$ is called minimal if for some maximal congruence $\theta$ witnessing the edge and every $a^{\prime}, b^{\prime} \in A$ such that $\left(a, a^{\prime}\right),\left(b, b^{\prime}\right) \in \theta$, we have $\operatorname{Sg}_{\mathbf{A}}\left(a^{\prime}, b^{\prime}\right)=\operatorname{Sg}_{\mathbf{A}}(a, b)$.

A witnessing congruence $\theta$ for an edge $(a, b)$ necessarily separates $a$ and $b$, i.e., $(a, b) \notin \theta$, since each congruence block of an idempotent algebra is a subuniverse. Moreover, if $\theta$ is a witness for an edge $(a, b)$, then any proper congruence of $\operatorname{Sg}_{\mathbf{A}}(a, b)$ containing $\theta$ witnesses the same edge.

Note that if $(a, b)$ is an edge of majority or abelian type, then so is $(b, a)$. If $(a, b)$ is a semilattice edge it can happen that $(b, a)$ is not an edge at all, in fact this is always the case for minimal edges in a minimal Taylor algebra. 
In order to make the concepts in this paper elegant and theorems more general, we deviate from the definition given in e.g. [18, 25]. There, majority edges have an additional requirement that the same congruence does not witness the semilattice type, and abelian edges (called affine) required the quotient to be an affine module. Also note that the definition of abelian edges (as well as the original affine edges) is of a different type: it restricts the set of term operations from above, as opposed to semillatice and majority edges that restrict them from below. We shall see in Theorem 5.12 that these differences disappear in minimal Taylor algebras.

Minimal edges do not appear in Bulatov's theory in this form. Somewhat related are thin edges, which at present have rather technical definitions with the exception of thin semilattice edges. We show in Proposition 5.14 that minimal semilattice edges and thin semilattice edges coincide in minimal Taylor algebras.

\section{Taylor algebras}

This section presents unifications, simplifications, and refinements of the three algebraic theories in the setting of Taylor algebras (still finite and idempotent) that are not necessarily minimal. In Subsection 4.1 we discuss the already existing refinements to the proof of the absorption theorem (and provide two additional new refinements in Proposition 4.2 and Proposition 4.4). This gives tight links to Zhuk's theory, in particular, centers and projective subuniverses. Subsection 4.2 contains the main contribution of this section, Theorem 4.7. This theorem together with additional technical contributions, Theorems 4.10 and 4.11 directly imply the fundamental facts in the two proofs of the CSP Dichotomy Theorem - the four types theorem and the connectivity theorem, discussed in Subsection 4.3. The proofs of claims in this section are in Appendix B

\subsection{Absorption theorem}

We phrase the absorption theorem in a slightly simplified form to keep the presentation compact.

Theorem 4.1 (Absorption Theorem). [3] If $\mathbf{A}$ is Taylor and $R \leq_{s d} \mathbf{A}^{2}$ is proper and linked, then $\mathbf{A}$ has a nontrivial absorbing subuniverse.

The original proof can be divided into 3 steps.

(1) From $\mathbf{A}$ being Taylor it is derived that $\mathbf{A}$ either has a nontrivial binary absorbing subuniverse or a transitive term operation $t$ of some arity $n$, i.e., for each $b, c \in A$ and every coordinate $i$ of $t$, there exists a tuple $\mathbf{a} \in A^{n}$ with $a_{i}=b$ such that $t(\mathbf{a})=c$.

(2) Using the transitive operation, it is proved that if $\mathbf{A}$ has no nontrivial absorbing subuniverses, then $R$ is left or right central.

(3) It is shown that the transitive operation witnesses that the left (right) center absorbs $\mathbf{A}$.

We now comment on subsequent improvements and simplifications.

The first step was explored in more detail in [7. Lemma 2.7. in [7] shows that each algebra has a nontrivial projective subuniverse or a transitive term operation. A simple argument then shows that every projective subuniverse in a Taylor algebra is 2-absorbing, a witness is, e.g., any operation of the form $t(x, \ldots, x, y, \ldots, y)$ where $t$ is cyclic.

As for the second step, it has turned out that left (or right) central relations can be very easily obtained from linked relations by means of pp-definitions, avoiding algebraic considerations altogether. We give a refined version that derives central relations with further properties.

Proposition 4.2. Let $R \subseteq s d A^{2}$ be linked and proper. Then $R$ pp-defines a subdirect proper central relation on $A$ which is symmetric or transitive.

The third step, that a transitive operation witnesses absorption of left centers, is straightforward. A significant refinement, Corollary 7.10.2 in [55. shows that left centers are, in fact, ternary absorbing. An adjustment of the proof will also help us in proving Theorem 5.9. 
Proposition 4.3. [55] If $B$ is a Taylor center of an algebra $\mathbf{A}$, then $B \unlhd_{3} \mathbf{A}$.

Note that, in the previous theorem $\mathbf{A}$ need not be a Taylor algebra, but $\mathbf{C}$ (where the witnessing relation is $\left.R \leq_{s d} \mathbf{A} \times \mathbf{C}\right)$ must be. The following proposition states that we can switch the condition:

Proposition 4.4. If $B$ is a center of a Taylor algebra $\mathbf{A}$, then $B \unlhd_{3} \mathbf{A}$.

In the remainder of the paper, the assumptions of the latter proposition are easier to satisfy the algebra $\mathbf{A}$ is usually Taylor by default.

Altogether, either of the propositions above provides the following improvement of the absorption theorem, which does not seem to be explicitly stated in the literature.

Corollary 4.5. If $\mathbf{A}$ is Taylor and $R \leq_{s d} \mathbf{A}^{2}$ proper and linked, then $\mathbf{A}$ has a nontrivial 3absorbing subuniverse.

\subsection{Subdirect irredundant subpowers}

We now present the unification result. It says that any "interesting" (subdirect irredundant proper) relation either pp-defines an interesting binary relation or pp-defines (it is even inter-pp-definable with) ternary relations of very particular shape - they are graphs of quasigroup operations.

Definition 4.6. A relation $R \subseteq A^{3}$ is called strongly functional if

- binary projections of $R_{i}$ are equal to $A^{2}$, and

- a tuple in $R_{i}$ is determined by values on any two coordinates.

Theorem 4.7. Let $R \subseteq$ sd $A^{n}$ be an irredundant proper relation. Then either

- $R$ pp-defines an irredundant and proper $R^{\prime} \subseteq$ sd $A^{2}$, or

- there exist strongly functional ternary relations $R_{1}, \ldots, R_{n} \subseteq$ sd $A^{3}$ such that the set $\left\{R_{1}, \ldots\right.$, $\left.R_{m}\right\}$ is inter-pp-definable with $R$ (i.e., the $R_{i}$ 's pp-define $R$ and, conversely, $R$ pp-defines all the $R_{i}$ 's).

Theorem 4.7 implies that every algebra $\mathbf{A}$ has at least one of the following properties of its invariant relations.

(1) A has no proper irredundant subdirect subpowers.

(2) A has a proper irredundant binary subdirect subpower.

(3) A has a ternary strongly functional subpower.

In the last case, it is easy to pp-define a congruence on $\mathbf{A}^{2}$ such that the diagonal is one of its blocks, so $\mathbf{A}$ is abelian in this case. If $\mathbf{A}$ is Taylor, Theorem 3.4 then gives a good understanding of $\mathbf{A}$ - it is an affine module.

Proposition 4.8. If $R \leq \mathbf{A}^{3}$ is a strongly functional relation, then $\mathbf{A}$ is abelian.

In case (1), subdirect relations have a very simple structure; for instance, any constraint $R\left(x_{1}, \ldots\right.$, $x_{n}$ ) with subdirect $R$ is effectively a conjunction of bijective dependencies $x_{i}=f\left(x_{j}\right)$. It is also immediate that $\mathbf{A}$ is polynomially complete, that is, every operation on $A$ is in the clone generated by $\mathbf{A}$ together with the constant operations. Indeed, polynomial completeness is equivalent to having no proper reflexive (that is, containing all the tuples $(a, a, \ldots, a))$ irredundant subpowers. Less trivially, case (1) often leads to majority edges, as we show in Theorem 4.10 below. However, we require the following definition first.

Definition 4.9. Let $\mathbf{A}$ be an algebra. By the connected-by-subuniverses equivalence, denoted $\mu_{\mathbf{A}}$, we mean the smallest equivalence containing all the pairs $(a, b)$ such that $\operatorname{Sg}_{\mathbf{A}}(a, b) \neq A$. 
We remark that the equivalence $\mu_{\mathbf{A}}$ is not, in general, a congruence of $\mathbf{A}$, so this concept may seem somewhat unnatural from the algebraic perspective.

Theorem 4.10. Suppose that $\mathbf{A}$ is simple and has no subdirect proper irredundant subpowers. Then there exists a term operation $t \in \mathrm{Clo}_{3}(\mathbf{A})$ such that for any $(a, b) \notin \mu_{\mathbf{A}}, t(a, a, b)=t(a, b, a)=$ $t(b, a, a)=a$.

In case (2) and when $\mathbf{A}$ is simple, a binary irredundant relation is necessarily linked. Then we get a central relation, e.g., by Proposition 4.2, and often also semilattice edges (please note the important, but easy-to-miss condition on the size of the algebra).

Theorem 4.11. Suppose $\mathbf{A}$ with $|A|>2$ is simple and there exists a proper irredundant subdirect binary subpower. Then there exists $\mu_{\mathbf{A}}$-class $B$ such that, for every $b \in B, a \notin B$, the pair $(a, b)$ is a semilattice edge witnessed by the identity congruence.

\subsection{Fundamental theorems of dichotomy proofs}

Zhuk's four types theorem is now a consequence of Theorem 4.7. Proposition 4.8, and Proposition 4.2. Indeed, one simply applies these facts to $\mathbf{A}$ factored by a maximal congruence, which is a simple algebra, and then lifts 2-absorbing subuniverses and centers back to $\mathbf{A}$.

Corollary 4.12. [The Four Types Theorem] Let $\mathbf{A}$ be an algebra, then

(a) A has a nontrivial 2-absorbing subuniverse, or

(b) A has a nontrivial center, (which is a Taylor center in the case that $\mathbf{A}$ is a Taylor algebra), or

(c) $\mathbf{A} / \alpha$ is abelian for some proper congruence $\alpha$ of $\mathbf{A}$, or

(d) $\mathbf{A} / \alpha$ is polynomially complete for some proper congruence $\alpha$ of $\mathbf{A}$.

In the introduction we referred to four types of subuniverses whereas cases (c) and (d) talk about congruences - the subuniverses used in [55] are obtained from blocks of such congruences.

Examples of simple Taylor algebras, for which one of the cases takes place and no other, are (a) a two-element semilattice, (b) a two-element majority algebra, (c) an affine Mal'cev algebra, and $(\mathrm{d})$ the three element rock-paper-scissors algebra

$$
\text { (\{paper, rock, scissors\}; winner }(x, y)) \text {. }
$$

Note, however, that Corollary 4.12 does not require that $\mathbf{A}$ is Taylor. If it is, then we get additional properties: centers are 3 -absorbing by Proposition 4.3 and abelian algebras are term equivalent to affine modules by Theorem 3.4. For non-Taylor idempotent algebras, [56] suggests a similar five type theorem, which also follows immediately from the presented results.

The connectivity theorem of Bulatov is also a straightforward consequence of the obtained results, Theorem 4.7, Proposition 4.8, Theorem 4.10, and Theorem 4.11. In fact, a little additional effort gives a stronger statement - for minimal edges instead of edges.

Corollary 4.13. [The Connectivity Theorem] The directed graph formed by the minimal edges of any algebra is connected.

Notice that the last theorem also does not require the algebra to be Taylor. Outside Taylor algebras, it makes sense to separate abelian edges into two types: affine that are the same as abelian edges in the Taylor case, and sets whose only term operations are projections, as is done in 25$]$. 


\section{Minimal Taylor algebras}

We start this section by recalling the central definition and giving some examples.

Definition 5.1. An algebra $\mathbf{A}$ is called a minimal Taylor algebra if it is Taylor but no proper reduct of $\mathbf{A}$ is.

Examples of minimal Taylor algebras include two-element semilattices, two-element majority algebras, and affine Mal'cev algebras. This follows from the description of their term operations: the term operations of the two-element semilattice $(\{0,1\} ; \vee)$ are exactly the operations of the form $x_{i_{1}} \vee x_{i_{2}} \vee \cdots \vee x_{i_{k}}$; the term operations of the two-element majority algebra $(\{0,1\} ;$ maj) are exactly the idempotent, monotone (i.e., compatible with the inequality relation $\leq$ ), and self-dual (i.e., compatible with the disequality relation $\neq$ ) operations; the term operations of an affine Mal'cev algebra over an abelian group are exactly the operations of the form $a_{1} x_{1}+a_{2} x_{2}+\cdots+a_{n} x_{n}$, where $a_{i}$ are integers that sum up to one. (Each of the mentioned facts is either simple or follows from 51.) In fact, there are exactly four minimal Taylor algebras on a two-element set: the two semilattices, the majority algebra and the two-element affine Mal'cev algebra.

A nice example of a minimal Taylor algebra on a three-element domain is the rock-paperscissors algebra mentioned after The Four Types Theorem. To see that this algebra is minimal Taylor observe that any term operation behaves on any two-element set like the term operation of a two-element semilattice with the same set of essential coordinates. Therefore, the original operation can be obtained by identifying variables in any term operation having at least two essential coordinates. The same argument shows that any semilattice, not necessarily two-element, is minimal Taylor.

In Subsection 5.1 we give the basic general theorems that were proved in [15] in the context of minimal bounded width algebras. Subsection 5.2 concentrates on absorption and related concepts in Zhuk's theory. It turns out that 2-absorbing sets are exactly projective subuniverses (Theorem 5.7) and 3-absorbing sets are exactly centers (Theorem 5.9). Subsection 5.3 shows that edges substantially simplify in minimal Taylor algebras (Theorem 5.12) and gives additional information for minimal edges; in particular, minimal semilattice edges coincide with thin semilattice edges as defined in 22, 25 (Proposition 5.14). Finally, in Subsection 5.4, we demonstrate a strong interaction between absorption and edges. We show that 2-absorbing subuniverses are exactly subsets that are, in some sense, stable under all the edges (Theorem 5.18), we provide somewhat weaker interaction between absorbing subuniverses and subsets stable under semilattice and abelian edges (Theorem [5.20), we give a common witnessing operation for all the edges as well as all the 2- and 3-absorbing subuniverses (Theorem 5.22), and we show that each such a witnessing operation generates the whole clone of term operations (Theorem 5.23). Proofs for this section (including verification of examples) are in Appendix C.

\subsection{General facts}

It is not immediate from the definitions that each Taylor algebra has a minimal Taylor reduct. Nevertheless, this fact easily follows from the characterization of Taylor algebras by means of cyclic operations.

Proposition 5.2. Every Taylor algebra has a minimal Taylor reduct.

Another simple, but important consequence of cyclic operations is the following proposition. The result is slightly more technical than most of the others, but it is in the core of many strong properties of minimal Taylor algebras.

Proposition 5.3. Let $\mathbf{A}$ be a minimal Taylor algebra and $B \subseteq A$ be closed under an operation $f \in \mathrm{Clo}(\mathbf{A})$ such that $B$ together with the restriction of $f$ to $B$ forms a Taylor algebra. Then $B$ is a subuniverse of $\mathbf{A}$.

A similar method based on cyclic operations prove that the class of minimal Taylor algebras is closed under the standard constructions. 
Proposition 5.4. Any subalgebra, finite power, or quotient of a minimal Taylor algebra is a minimal Taylor algebra.

\subsection{Absorption}

The goal of this section is to show that absorbing subsets, which are abundant in general Taylor algebras by Corollary 4.12 and Proposition 4.4, have strong properties in minimal Taylor algebras. We start with a surprising fact, which clearly fails in general Taylor algebras.

Theorem 5.5. Let $\mathbf{A}$ be a minimal Taylor algebra and $B$ an absorbing set of $\mathbf{A}$. Then $B$ is a subuniverse of $\mathbf{A}$.

Now we move on to 2-absorption. We have already mentioned in Subsection 4.1 that projectivity is a stronger form of absorption in Taylor algebras, but we can go even further.

Definition 5.6. Let $\mathbf{A}$ be an algebra and $B \subseteq A$. The set $B$ is a strongly projective subuniverse of $\mathbf{A}$ if for every $f \in \mathrm{Clo}_{n}(\mathbf{A})$ and every essential coordinate $i$ of $f$, we have $f(\mathbf{a}) \in B$ whenever $\mathbf{a} \in A^{n}$ is such that $a_{i} \in B$.

The property of being a strong projective subuniverse is indeed very strong. For example, in any non-trivial clone, strong projective subuniverse is 2-absorbing and every binary operation of the clone, except for projections, witnesses the absorption. The next theorem states that strong projectivity in minimal Taylor algebras is equivalent to 2-absorption, which in general is a much weaker concept.

Theorem 5.7. The following are equivalent for any minimal Taylor algebra $\mathbf{A}$ and a set $B \subseteq A$.

(a) B 2-absorbs A.

(b) $R(x, y, z)=B(x) \vee B(y) \vee B(z)$ is a subuniverse of $\mathbf{A}^{3}$.

(c) $B$ is a projective subuniverse of $\mathbf{A}$

(d) $B$ is a strongly projective subuniverse of $\mathbf{A}$.

The main value of this theorem is the implication showing that, in minimal Taylor algebras, every 2absorption, i.e. (a), is as strong as possible (d). Moreover (c) provides a nice relational description of 2-absorption, which collapses the general condition from Proposition 3.8 for projectivity to arity 3. We note that none of the conditions are equivalent in Taylor algebras without the minimality assumption.

The following proposition collects some strong and unusual properties of 2-absorbing subuniverses in minimal Taylor algebras. Already the first item might be surprising since the union of two subuniverses is rarely a subuniverse.

Proposition 5.8. Let $\mathbf{A}$ be a minimal Taylor algebra and $B \unlhd_{2} \mathbf{A}$.

1. If $C \leq \mathbf{A}$ then $B \cup C \leq \mathbf{A}$.

2. If $C$ is proper and $C \unlhd \mathbf{A}$ by $f$, then

(a) $B \cup C \unlhd \mathbf{A}$ by $f$, and

(b) $B \cap C \neq \emptyset$ and $B \cap C \unlhd \mathbf{A}$ by $f$.

3. Every minimal Taylor algebra A has a unique minimal 2-absorbing subalgebra B. Moreover, this algebra $\mathbf{B}$ does not have any nontrivial 2-absorbing subuniverse.

As for absorption of higher arity, we have already shown in Proposition 4.4 that centers are 3-absorbing. Next theorem says that, in minimal Taylor algebras, the converse is true as well.

Theorem 5.9. The following are equivalent for any minimal Taylor algebra $\mathbf{A}$ and a set $B \subseteq A$. 
(a) B 3-absorbs $\mathbf{A}$.

(b) $R(x, y)=B(x) \vee B(y)$ is a subuniverse of $\mathbf{A}^{2}$.

(c) B is a (Taylor) center of $\mathbf{A}$.

(d) there exists $\mathbf{C}$ with $\mathrm{Clo}(\mathbf{C}) \subseteq \mathrm{Clo}(\{0,1\}$; maj) such that $R(x, y)=B(x) \vee(y=0)$ is a centrality witness.

Moreover, if $B=\{b\}$, then these items are equivalent to

(e) B absorbs $\mathbf{A}$.

Just like in Theorem 5.7 we have that a relatively weak notion of 3-absorption implies a very strong type of centrality which is (d). Let us investigate (d) in greater detail. To every operation of $\mathbf{A}$, say $f$, we associate an operation $f^{\prime} \in \operatorname{Clo}\left(\{0,1\}\right.$, maj) such that $f(\mathbf{a}) \in B$ whenever $f^{\prime}(\mathbf{x})=1$ and $\mathbf{x}$ is the characteristic tuple of $\mathbf{a}$ with respect to $B$ (i.e. $x_{i}=1$ if and only if $a_{i} \in B$ ). That is, from the viewpoint of "being outside $B$ " vs. "being inside $B$ " every operation outputs "inside $B$ " every time the corresponding operation of $\operatorname{Clo}(\{0,1\}$; maj) outputs 1 .

In fact, there exists a cyclic $t$ in $\mathbf{A}$ (say, $p$-ary) such that $R(x, y)=B(x) \vee(y=0)$ is a sub-

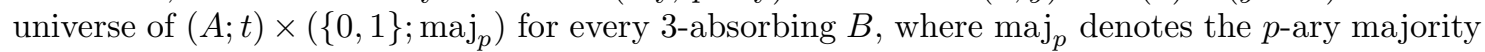
function. This translates to a simpler statement: for every 3 -absorbing $B$ we have $t(\mathbf{a}) \in B$ whenever majority of the $a_{i}$ belong to $B$, and we cannot expect more, as witnessed by the 2element majority algebra. Since $t$ is cyclic it generates the whole clone and, for example, 3 ) in Proposition 5.11 below becomes obvious.

Item (b) provides a relational description of 3-absorption, while item (c) provides a connection with the notion of center (whether it is Taylor or not). We now give an example that (e) and (a) are not equivalent even in minimal Taylor algebras if $B$ has more than one element.

Example 5.10. Consider the algebra $\mathbf{A}=(\{0,1,2\}, m)$ where $m$ is the majority operation such that $m(a, b, c)=a$ whenever $|\{a, b, c\}|=3$. This algebra is minimal Taylor and the set $C=\{0,1\}$ is an absorbing subuniverse of $\mathbf{A}$. However, $C$ is not a center of $\mathbf{A}$.

Finally, we list some strong and unusual properties of 3-absorbing subuniverses. They are not as strong as in the case of 2-absorbing subuniverses, which is to be expected since every 2-absorbing subuniverse is 3 -absorbing but not vice versa.

Proposition 5.11. Let $\mathbf{A}$ be a minimal Taylor algebra and $B, C \unlhd_{3} \mathbf{A}$.

1. $B \cup C \leq \mathbf{A}$

2. If $B \cap C \neq \emptyset$ then $B \cap C \unlhd_{3} \mathbf{A}$.

3. If $B \cap C=\emptyset$ then $B^{2} \cup C^{2}$ is a congruence on the algebra with universe $B \cup C$ and the quotient is term-equivalent to a two-element majority algebra.

\section{$5.3 \quad$ Edges}

The next theorem says that, in minimal Taylor algebras, every "thick" edge, in the terminology of [22, 23], is automatically a subuniverse. This property is a simple consequence of the result we have already stated, whereas it was relatively painful to achieve using the original approach. We additionally obtain that semilattice and majority edges have unique witnessing congruences.

Theorem 5.12. Let $(a, b)$ be an edge (semilattice, majority, or abelian) of a minimal Taylor algebra $\mathbf{A}$ and $\theta$ a witnessing congruence of $\mathbf{E}=\operatorname{Sg}_{\mathbf{A}}(a, b)$.

(a) If $(a, b)$ is a semilattice edge, then $\mathbf{E} / \theta$ is term equivalent to a two-element semilattice with absorbing element $b / \theta$. 
(b) If $(a, b)$ is a majority edge, then $\mathbf{E} / \theta$ is term equivalent to a two-element majority algebra.

(c) if $(a, b)$ is an abelian edge, then $\mathbf{E} / \theta$ is term equivalent to an affine Mal'cev algebra of an abelian group isomorphic to $\mathbb{Z} / p_{1}^{k_{1}} \times \cdots \times \mathbb{Z} / p_{i}^{k_{i}}$ for distinct primes $p_{1}, \ldots, p_{i}$ and positive integers $k_{1}, \ldots, k_{i}$, where $\mathbb{Z} / m$ denotes the group of integers modulo $m$.

Moreover a semillatice edge is witnessed by exactly one congruence of $\mathbf{E}$, and that congruence is maximal. The same holds for majority edges.

For minimal edges we can say a bit more. If $(a, b)$ is a minimal edge witnessed by $\theta$, a congruence on $\mathbf{E}=\operatorname{Sg}_{\mathbf{A}}(a, b)$, then $\mathbf{E} / \theta$ is simple. In particular, for abelian edges, $\mathbf{E} / \theta$ is an affine Mal'cev algebra of a group isomorphic to $\mathbb{Z} / p$. Moreover, such an $\mathbf{E}$ has a unique maximal congruence as shown in the next proposition. This implies that the type of a minimal edge is unique and so is the direction of a semilattice minimal edge and the prime $p$ associated to an abelian minimal edge.

Proposition 5.13. Let $(a, b)$ be a minimal edge in a minimal Taylor algebra. Then $\mathbf{E}=\operatorname{Sg}_{\mathbf{A}}(a, b)$ has a unique maximal congruence equal to $\mu_{\mathbf{E}}$. In particular, minimal edges have unique types.

The structure of minimal semilattice edges is especially simple.

Proposition 5.14. Let $(a, b)$ be a minimal semilattice edge in a minimal Taylor algebra. Then $\{a, b\}$ is a subuniverse of $\mathbf{A}$, so $\operatorname{Sg}_{\mathbf{A}}(a, b)=\{a, b\}$ and the witnessing congruence is the equality.

Unfortunately, majority and abelian edges do not simplify in a similar way; see Example 5.15 and Example [5.16. Weaker versions of Proposition 5.14 have been developed by Bulatov (comp. Lemma 12 and Corollary 13 in [22]) to deal with this problem.

Example 5.15. Let $A=\{0,1,2,3\}$ and $\alpha$ the equivalence relation on $A$ with blocks $\{0,2\}$ and $\{1,3\}$. Define a symmetric ternary operation $g$ on $A$ as follows. When two of the inputs to $g$ are equal, $g$ is given by $g(a, a, a+1)=a, g(a, a, a+2)=g(a, a, a+3)=a+2$ (all modulo 4) and when all three inputs to $g$ are distinct, $g$ is given by $g(a, b, c)=d-1(\bmod 4)$ where $a, b, c, d$ are any permutation of $0,1,2,3$. Then $\mathbf{A}=(A ; g)$ is a minimal Taylor algebra, $\alpha$ is a congruence on $\mathbf{A}$, and each of pair of elements in different $\alpha$-blocks is a minimal majority edge where the witnessing congruence is equal to $\alpha$.

Example 5.16. Let $\mathbf{A}=(\{a, b, c, d\}, p)$, where $p$ is a Mal'cev operation with the following properties. The operation $p$ commutes with the permutations $\sigma=(a c)$ and $\tau=(b d)$. The polynomials $+_{a}=p(\cdot, a, \cdot),+_{b}=p(\cdot, b, \cdot)$ define abelian groups:

\begin{tabular}{c|cccc}
$++_{a}$ & $a$ & $b$ & $c$ & $d$ \\
\hline$a$ & $a$ & $b$ & $c$ & $d$ \\
$b$ & $b$ & $c$ & $d$ & $a$ \\
$c$ & $c$ & $d$ & $a$ & $b$ \\
$d$ & $d$ & $a$ & $b$ & $c$
\end{tabular}

\begin{tabular}{c|cccc}
$+b$ & $a$ & $b$ & $c$ & $d$ \\
\hline$a$ & $b$ & $a$ & $d$ & $c$ \\
$b$ & $a$ & $b$ & $c$ & $d$ \\
$c$ & $d$ & $c$ & $b$ & $a$ \\
$d$ & $c$ & $d$ & $a$ & $b$
\end{tabular}

Then $\mathbf{A}$ is a minimal Taylor algebra, with a unique maximal congruence $\theta$ whose congruence classes are $\{a, c\}$ and $\{b, d\}$. Each pair of elements of $\mathbf{A}$ in different congruence classes of $\theta$ is a minimal abelian edge of $\mathbf{A}$ such the witnessing congruence is $\theta$.

We can also provide nontrivial information about $\operatorname{Sg}(a, b)$ in case that $(a, b)$ is not necessarily an edge, and this information helps in proving Theorem 5.23 in the next subsection (and shows that case (d) in Corollary 4.12 is never necessary for two-generated algebras). However, the following fundamental question remains open: Does there exist a minimal Taylor algebra such that, for some $a, b$, neither $(a, b)$ nor $(b, a)$ is an edge? 


\subsection{Absorption and edges}

We start this subsection with a definition that will connect absorption with edges.

Definition 5.17. Let $\mathbf{A}$ be an algebra, let $B \subseteq A$ and let $(b, a)$ be an edge. We say that $B$ is stable under $(b, a)$ if, for every witnessing congruence $\theta$ of $\operatorname{Sg}_{\mathbf{A}}(b, a)$ such that $b / \theta$ intersects $B$, each $\theta$-block intersects $B$.

As the next theorem states, stability under every edge can be added as a next item to Theorem 5.7. This direct connection of absorption, which is a global property, to local concepts in Bulatov's theory is among the most surprising phenomena that the authors have encountered in this work.

Theorem 5.18. The following are equivalent for any minimal Taylor algebra $\mathbf{A}$ and a set $B \subseteq A$.

(a) B 2-absorbs A.

(b) $B$ is stable under all the edges.

The implication from (b) to (a) does not require the full strength of stability for semilattice and majority edges. It is enough to require that for a minimal semilattice or a majority edge $(b, a)$ it is never the case that $b / \theta \subseteq B$ and $a / \theta \cap B=\emptyset$, where $\theta$ is the edge-witnessing congruence of $\operatorname{Sg}(b, a)$ (which is the equality relation on $\{a, b\}$ in case of semilattice edges). The following example shows that stability under abelian edges cannot be significantly weakened.

Example 5.19. We consider the four-element algebra $\mathbf{A}=(\{0,1,2, *\}, \cdot)$ with binary operation . given by

\begin{tabular}{c|cccc}
$\cdot$ & 0 & 1 & 2 & $*$ \\
\hline 0 & 0 & 2 & 1 & $*$ \\
1 & 2 & 1 & 0 & 2 \\
2 & 1 & 0 & 2 & 1 \\
$*$ & $*$ & 2 & 1 & $*$
\end{tabular}

Then A is a minimal Taylor algebra, with a semilattice edge $(0, *)$, with $\{0,1,2\}$ an affine subalgebra, and with a congruence $\theta$ corresponding to the partition $\{0, *\},\{1\},\{2\}$ such that $\mathbf{A} / \theta$ is affine. The set $\{*\}$ is stable under semilattice and majority edges and there is no minimal abelian edge $(*, a)$ with $a \neq *$. But $\{*\}$ is not an absorbing subalgebra of $\mathbf{A}$.

For absorption of higher arity the connection to edges is not as tight as for 2-absorption. Nevertheless, one direction still works and both directions work for singletons.

Theorem 5.20. Any absorbing set of a minimal Taylor algebra $\mathbf{A}$ is stable under semilattice and abelian edges. Moreover, for any $b \in A$ the following are equivalent.

(a) $\{b\}$ absorbs $\mathbf{A}$

(b) $\{b\}$ is stable under semilattice and abelian edges.

Stability under semilattice edges for the implication from (b) to (a) can be again replaced by the requirement that there is no minimal semilattice edge $(b, a)$ with $b \in B$ and $a \notin B$. Example 5.19 shows that this is not the case for abelian edges.

The following example shows that the implication from (b) to (a) does not hold for nonsingleton subuniverses.

Example 5.21. Consider the algebra $\mathbf{A}=(\{0,1,2\}, m)$ where $m$ is the majority operation such that $m(a, b, c)=2$ whenever $|\{a, b, c\}|=3$. This algebra is minimal Taylor, every pair of distinct elements forms a subuniverse, and every pair is a minimal majority edge. So there are no semilattice or abelian edges. However, the subuniverse $\{0,1\}$ is not absorbing. 
An important fact for the edge approach is that semilattice, majority, and Mal'cev operations coming from edges can be unified, see Theorem 7 in 22 . In minimal Taylor algebras, a simple consequence of the already stated results is that we not only have a common ternary witness for all the edges but also for all the binary and ternary absorptions.

Theorem 5.22. Every minimal Taylor algebra A has a ternary term operation $f$ such that if $(a, b)$ is an edge witnessed by $\theta$ on $\mathbf{E}=\operatorname{Sg}_{\mathbf{A}}(a, b)$, then

- if $(a, b)$ is a semilattice edge, then $f(x, y, z)=x \vee y \vee z$ on $\mathbf{E} / \theta$ (where $b / \theta$ is the top);

- if $(a, b)$ is a majority edge, then $f$ is the majority operation on $\mathbf{E} / \theta$ (which has two elements);

- if $(a, b)$ is an abelian edge, then $f(x, y, z)=x-y+z$ on $\mathbf{E} / \theta$;

- $f$ witnesses all the ternary absorptions $B \unlhd_{3} \mathbf{A}$;

- any binary operation obtained from $f$ by identifying two arguments witnesses all the binary absorptions $B \unlhd_{2}$ A.

In fact, any ternary operation $f$ defined from a cyclic term operation $t$ of odd arity $p \geq 3$ by

$$
f(x, y, z)=t(\underbrace{x, x, \ldots, x}_{k \times}, \underbrace{y, y, \ldots, y}_{l \times}, \underbrace{z, z, \ldots, z}_{m \times}),
$$

where $k+l, l+m, k+m>p / 2$, satisfies all the items in Theorem 5.22 except possibly the third one (which can be obtained by picking $k, l$, and $m$ a bit more carefully).

We finish this section with a theorem stating that any ternary witness of edges generates the whole clone of the algebra. In particular, the number of minimal Taylor clones on a domain of size $n$ is at most $n^{n^{3}}$.

Theorem 5.23. If $\mathbf{A}$ is a minimal Taylor algebra, then $\operatorname{Clo}(A ; f)=\operatorname{Clo}(\mathbf{A})$ for any operation $f$ satisfying the first three items in Theorem 5.22.

\section{$6 \quad$ Omitting types}

In this section we consider classes of algebras whose graph only contains edges of certain types. We say that an algebra is a-free if it has no abelian edges. More generally, an algebra is $\mathrm{x}$-free or is $\mathrm{xy}$-free, where $\mathrm{x}, \mathrm{y} \in\{(\mathrm{a})$ belian, (m)ajority, (s)emilattice $\}$ if it has no edges of type $\mathrm{x}$ (of types $\mathrm{x}, \mathrm{y})$.

It turns out that within minimal Taylor algebras these "omitting types" conditions are often equivalent to important properties of algebras. In the theorems below we prove the equivalence of the following four types of conditions: (i) the absence of edges of a certain type (equivalently, minimal edges of the same type); (ii) properties of absorption and the four types in Zhuk's approach; (iii) the existence of a certain special term operations; (iv) algorithmic properties of the CSP. Here recall that the properties of "having bounded width" and "having few subpowers" characterize the applicability of the two basic algorithmic ideas in the CSP - local propagation algorithms [29, 4] and finding a generating set of all solutions [11, 40. Theorems in this section are consequences of the theory we have already built in the previous section and known results (see [8]). The proofs can be found in Appendix D.

The first theorem concerns the class of algebras omitting abelian edges. Numerous characterizations of this class are known for general algebras and we do not add a new one, but we state the characterization for comparison with the other classes. In order to state a characterization in terms of identities we recall that an operation $f$ is a weak near unanimity operation (or wnu for short) if it satisfies $f(y, x, \ldots, x)=f(x, y, x, \ldots, x)=\cdots=f(x, \ldots, x, y)$ for every $x, y$ in the algebra.

Theorem 6.1. The following are equivalent for any algebra $\mathbf{A}$. 
(i) $\mathbf{A}$ is a-free.

(ii) No subalgebra of $\mathbf{A}$ falls into case (c) in Corollary 4.12, i.e., no subalgebra of $\mathbf{A}$ has a nontrivial affine quotient.

(iii) A has a wnu term operation of every arity $n \geq 3$.

(iv) A has bounded width.

Minimal Taylor algebras omitting other types of edges do have significantly stronger properties than general Taylor algebras omitting those edges. Minimal s-free algebras are exactly those for which option (a) in Corollary 4.12 does not hold, and that have the few subpowers property 23. The few subpowers property, i.e., that the number of subuniverses of $\mathbf{A}^{n}$ is $2^{O(n)}$, can be characterized by the existence of an edge term operation [11] in general. In minimal Taylor algebras, the second strongest edge operation always exists - the 3-edge operation defined by the identities $e(y, y, x, x)=e(y, x, y, x)=e(x, x, x, y)=x$. This is significant, because the exponent in the running time of the few subpowers algorithm depends on the least $k$ such that the algebra has a $k$-edge term operation. The number 3 here is best possible: a 2 -edge operation is the same as a Mal'cev operation appearing in Theorem 6.6.

Theorem 6.2. The following are equivalent for any minimal Taylor algebra $\mathbf{A}$.

(i) $\mathbf{A}$ is s-free.

(ii) Case (a) in Corollary 4.12 does not hold, that is, no subalgebra of $\mathbf{A}$ has a nontrivial 2absorbing subuniverse.

(iii) A has a 3-edge term operation.

(iv) A has few subpowers.

For the remaining omitting-single-type condition, m-freeness, we do not provide a natural condition in terms of identities, and we are not aware of algorithmic implications of this condition. Nevertheless, it can be characterized by means of absorption.

Theorem 6.3. The following are equivalent for any minimal Taylor algebra $\mathbf{A}$.

(i) $\mathbf{A}$ is $\mathbf{m}$-free.

(ii) Every center (3-absorbing subuniverse of) $\mathbf{B} \leq \mathbf{A}$ 2-absorbs $\mathbf{B}$, i.e., (b) implies (a) in Corollary 4.12 in all the subalgebras of $\mathbf{A}$.

(ii') Every subalgebra of $\mathbf{A}$ has a unique minimal 3-absorbing subuniverse

Surprisingly, if along with m-freeness we also limit the type of abelian edges allowed in an algebra, the resulting condition is equivalent to the existence of a binary commutative term operation. This is interesting, since the existence of a commutative term operation was not considered to be a natural requirement for the CSP (see the discussion in 8]) or in Universal Algebra. We call an abelian edge $(a, b)$ a $\mathbb{Z} / 2$-edge if the corresponding affine Mal'cev algebra $\operatorname{Sg}(a, b) / \theta$ is isomorphic to the affine Mal'cev algebra of $\mathbb{Z} / 2$.

Theorem 6.4. The following are equivalent for any minimal Taylor algebra $\mathbf{A}$.

(i) $\mathbf{A}$ is $\mathrm{m}$-free and has no $\mathbb{Z} / 2$-edges.

(iii) A has a binary commutative term operation

(iii') $\operatorname{Clo}(\mathbf{A})$ can be generated by a collection of binary operations. 
Properties of minimal Taylor algebras having edges of only one type can be derived as conjunctions of the properties stated above. For two of these cases, sm-free and as-free, we provide additional information.

Minimal Taylor am-free algebras are exactly those which have wnu operations of every arity $n \geq$ 2. These are exactly the minimal spirals in the terminology of [15] and a significant property is that for every $(a, b)$ such that neither $(a, b)$ nor $(b, a)$ is a minimal semilattice edge, there is a surjective homomorphism from $\operatorname{Sg}\{a, b\}$ onto the (three-element) free semilattice on two generators.

The sm-free minimal Taylor algebras are those where cases (a) and (b) in Corollary 4.12 do not occur. Additionally, these are exactly the hereditarily absorption free algebras studied in [8] and, also, the algebras with a Mal'cev term operation - a type of operation that played a significant role in the CSP [17].

Theorem 6.5. The following are equivalent for any minimal Taylor algebra $\mathbf{A}$.

(i) $\mathbf{A}$ is sm-free.

(ii) No subalgebra of $\mathbf{A}$ has a nontrivial absorbing subuniverse.

(iii) A has a Mal'cev term operation.

Finally, the as-free algebras are those where cases (a) and (c) in Corollary 4.12 do not occur and those that have bounded width and few subpowers. It is known [39, 11] that the latter property in general implies having a near-unanimity term operation of some arity. Surprisingly, in minimal Taylor algebras, the arity goes down directly to three. In the algorithmic language, these algebras have strict width two 35,8 .

Theorem 6.6. The following are equivalent for any minimal Taylor algebra $\mathbf{A}$.

(i) $\mathbf{A}$ is as-free.

(iii) A has a near unanimity term operation.

(iii') A has a majority term operation.

\section{Conclusion}

We have introduced the concept of minimal Taylor algebras and used it to significantly unify, simplify, and extend the three main algebraic approaches to the CSP - via absorption, via four types, and via edges. We believe that the theory started in this paper will help in attacking further open problems in computational complexity of CSP-related problems and Universal Algebra. There are, however, many directions which call for further exploration.

First, several technical questions naturally arise from the presented results: Do every two elements of a minimal Taylor algebra form an edge? How to characterize sets stable under affine and semilattice edges in a global way? Is it possible to characterize (3-)absorption in terms of edges? Does stability under other edge-types correspond to a global property? Is every minimal bounded width algebra a minimal Taylor algebra? Are the equivalent characterizations in Theorem6.3 equivalent to "every subalgebra has a unique minimal absorbing (rather than 3-absorbing) subuniverse"?

Second, both CSP dichotomy proofs 24, 55] require and develop more advanced Commutator Theory 36, 44 concepts and results, while in this paper we have merely used some fundamental facts about the basic concept, the abelian algebra. Is it possible to develop our theory in this direction as well, potentially providing sufficient tools for the dichotomy result? Also, is there a natural concept that would replace thin edges in Bulatov's approach?

Third, Brady in 15 provided a complete classification of minimal bounded width algebras of small size. Can such a detailed analysis be made also for minimal Taylor algebras? Is it possible to develop a strong theory or even full classification for minimal algebras in other classes, such as the algebras conjectured to characterize CSPs in log-space or nondeterministic log-space? 
Fourth, which of the facts presented in the paper have their counterpart for non-minimal Taylor algebras or even general finite idempotent algebras? Here we would like to mention Ross Willard's work (unpublished) that provides a generalization for some of the advanced facts in Zhuk's approach.

Finally, there is yet another, older, and highly developed theory of finite algebras, the Tame Congruence Theory started in [39]. What are the connections to the theory initiated in this paper?

\section{References}

[1] Libor Barto. Finitely related algebras in congruence modular varieties have few subpowers. J. Eur. Math. Soc. (JEMS), 20(6):1439-1471, 2018.

[2] Libor Barto and Alexandr Kazda. Deciding absorption. International Journal of Algebra and Computation, 26(05):1033-1060, 2016.

[3] Libor Barto and Marcin Kozik. Absorbing Subalgebras, Cyclic Terms, and the Constraint Satisfaction Problem. Logical Methods in Computer Science, Volume 8, Issue 1, February 2012 .

[4] Libor Barto and Marcin Kozik. Constraint satisfaction problems solvable by local consistency methods. J. ACM, 61(1):3:1-3:19, 2014.

[5] Libor Barto and Marcin Kozik. Robustly solvable constraint satisfaction problems. SIAM J. Comput., 45(4):1646-1669, 2016.

[6] Libor Barto and Marcin Kozik. Absorption in universal algebra and CSP. In The Constraint Satisfaction Problem: Complexity and Approximability, pages 45-77. 2017.

[7] Libor Barto, Marcin Kozik, and David Stanovský. Mal'tsev conditions, lack of absorption, and solvability. Algebra Universalis, 74(1):185-206, Sep 2015.

[8] Libor Barto, Andrei A. Krokhin, and Ross Willard. Polymorphisms, and how to use them. In The Constraint Satisfaction Problem: Complexity and Approximability, pages 1-44. 2017.

[9] Libor Barto, Jakub Opršal, and Michael Pinsker. The wonderland of reflections. Israel J. Math., 223(1):363-398, 2018.

[10] Clifford Bergman. Universal algebra, volume 301 of Pure and Applied Mathematics (Boca Raton). CRC Press, Boca Raton, FL, 2012. Fundamentals and selected topics.

[11] Joel Berman, Paweł Idziak, Petar Marković, Ralph McKenzie, Matthew Valeriote, and Ross Willard. Varieties with few subalgebras of powers. Trans. Amer. Math. Soc., 362(3):1445$1473,2010$.

[12] Manuel Bodirsky, Barnaby Martin, and Antoine Mottet. Discrete temporal constraint satisfaction problems. J. ACM, 65(2), February 2018.

[13] V. G. Bodnarčuk, L. A. Kalužnin, V. N. Kotov, and B. A. Romov. Galois theory for Post algebras. I, II. Kibernetika (Kiev), 3(5):1-10, 1969.

[14] Ferdinand Börner, Andrei A. Bulatov, Hubie Chen, Peter Jeavons, and Andrei A. Krokhin. The complexity of constraint satisfaction games and QCSP. Inf. Comput., 207(9):923-944, 2009 .

[15] Zarathustra Brady. Examples, counterexamples, and structure in bounded width algebras, 2020. 
[16] Joshua Brakensiek and Venkatesan Guruswami. Promise constraint satisfaction: Structure theory and a symmetric boolean dichotomy. In Proceedings of the Twenty-Ninth Annual ACMSIAM Symposium on Discrete Algorithms, SODA 2018, New Orleans, LA, USA, January 7-10, 2018, pages 1782-1801, 2018.

[17] Andrei Bulatov and Víctor Dalmau. A simple algorithm for Mal'tsev constraints. SIAM J. Comput., 36(1):16-27, July 2006.

[18] Andrei A. Bulatov. A graph of a relational structure and constraint satisfaction problems. In LICS, pages 448-457, 2004.

[19] Andrei A. Bulatov. A dichotomy theorem for constraint satisfaction problems on a 3-element set. J. ACM, 53(1):66-120, January 2006.

[20] Andrei A. Bulatov. Complexity of conservative constraint satisfaction problems. ACM Trans. Comput. Logic, 12(4), July 2011.

[21] Andrei A. Bulatov. The complexity of the counting constraint satisfaction problem. J. ACM, $60(5): 34: 1-34: 41,2013$.

[22] Andrei A. Bulatov. Graphs of finite algebras, edges, and connectivity. arXiv, abs/1601.07403, 2016.

[23] Andrei A. Bulatov. Graphs of relational structures: restricted types. In LICS, pages 642-651, 2016.

[24] Andrei A. Bulatov. A dichotomy theorem for nonuniform CSPs. In 58th IEEE Annual Symposium on Foundations of Computer Science, FOCS 2017, Berkeley, CA, USA, October 15-17, 2017, pages 319-330, 2017.

[25] Andrei A. Bulatov. Local structure of idempotent algebras I. arXiv, abs/2006.09599, 2020.

[26] Andrei A. Bulatov and Víctor Dalmau. Towards a dichotomy theorem for the counting constraint satisfaction problem. Inf. Comput., 205(5):651-678, 2007.

[27] Andrei A. Bulatov, Peter Jeavons, and Andrei A. Krokhin. Classifying the complexity of constraints using finite algebras. SIAM J. Comput., 34(3):720-742, 2005.

[28] Andrei A. Bulatov and Peter G. Jeavons. Algebraic structures in combinatorial problems. Technical Report MATH-AL-4-2001, Technische universität Dresden, Dresden, Germany, 2001.

[29] Andrei A. Bulatov, Andrei A. Krokhin, and Benoit Larose. Dualities for constraint satisfaction problems. In Complexity of Constraints - An Overview of Current Research Themes [Result of a Dagstuhl Seminar]., pages 93-124, 2008.

[30] Jakub Bulín, Andrei A. Krokhin, and Jakub Oprsal. Algebraic approach to promise constraint satisfaction. In Proceedings of the 51st Annual ACM SIGACT Symposium on Theory of Computing, STOC 2019, Phoenix, AZ, USA, June 23-26, 2019, pages 602-613, 2019.

[31] Catarina Carvalho, Barnaby Martin, and Dmitriy Zhuk. The complexity of quantified constraints using the algebraic formulation. In $42 n d$ International Symposium on Mathematical Foundations of Computer Science, MFCS 2017, August 21-25, 2017 - Aalborg, Denmark, pages 27:1-27:14, 2017.

[32] Béla Csákány. All minimal clones on the three-element set. Acta cybernetica, 6(3):227-238, 1983.

[33] R. Dechter. Constraint processing. Morgan Kaufmann Publishers, 2003. 
[34] T. Feder and M.Y. Vardi. Monotone monadic SNP and constraint satisfaction. In Proceedings of 25th ACM Symposium on the Theory of Computing (STOC), pages 612-622, 1993.

[35] T. Feder and M.Y. Vardi. The computational structure of monotone monadic SNP and constraint satisfaction: A study through datalog and group theory. SIAM Journal of Computing, 28:57-104, 1998.

[36] Ralph Freese and Ralph McKenzie. Commutator theory for congruence modular varieties, volume 125 of London Mathematical Society Lecture Note Series. Cambridge University Press, Cambridge, 1987.

[37] David Geiger. Closed systems of functions and predicates. Pacific J. Math., 27:95-100, 1968.

[38] P. Hell and Nešetřil. Graphs and homomorphisms, volume 28 of Oxford Lecture Series in Mathematics and its Applications. Oxford University Press, 2004.

[39] David Hobby and Ralph McKenzie. The structure of finite algebras, volume 76 of Contemporary Mathematics. American Mathematical Society, Providence, RI, 1988.

[40] Pawel M. Idziak, Petar Markovic, Ralph McKenzie, Matthew Valeriote, and Ross Willard. Tractability and learnability arising from algebras with few subpowers. SIAM J. Comput., 39(7):3023-3037, 2010.

[41] Peter Jeavons, David A. Cohen, and Marc Gyssens. Closure properties of constraints. J. ACM, 44(4):527-548, 1997.

[42] P.G. Jeavons. On the algebraic structure of combinatorial problems. Theoretical Computer Science, 200:185-204, 1998.

[43] Alexandr Kazda and Dmitriy Zhuk. Existence of cube terms in finite algebras. Algebra Universalis, 82(1):11, 2021.

[44] Keith A. Kearnes and Emil W. Kiss. The shape of congruence lattices. Mem. Amer. Math. Soc., 222(1046):viii+169, 2013.

[45] Keith A Kearnes and Ágnes Szendrei. Cube term blockers without finiteness. Algebra universalis, 78(4):437-459, 2017.

[46] Marcin Kozik, Andrei Krokhin, Matt Valeriote, and Ross Willard. Characterizations of several Maltsev conditions. Algebra Universalis, 73(3-4):205-224, 2015.

[47] Andrei A. Krokhin and Stanislav Zivny. The complexity of valued CSPs. In The Constraint Satisfaction Problem: Complexity and Approximability, pages 233-266. 2017.

[48] Benoît Larose and László Zádori. Taylor terms, constraint satisfaction and the complexity of polynomial equations over finite algebras. Int. J. Algebra Comput., 16(3):563-582, 2006.

[49] Petar Marković, Miklós Maróti, and Ralph McKenzie. Finitely related clones and algebras with cube terms. Order, 29(2):345-359, Jul 2012.

[50] Miklós Maróti and Ralph McKenzie. Existence theorems for weakly symmetric operations. Algebra Universalis, 59(3-4):463-489, 2008.

[51] Emil L. Post. The Two-Valued Iterative Systems of Mathematical Logic. (AM-5). Princeton University Press, 1941.

[52] Ivo Rosenberg. Über die funktionale Vollständigkeit in den mehrwertigen Logiken. Struktur der Funktionen von mehreren Veränderlichen auf endlichen Mengen. Rozpravy Československé Akad. Věd Řada Mat. Př́rod. Věd, 80(4):93, 1970. 
[53] Thomas J. Schaefer. The complexity of satisfiability problems. In Proceedings of the Tenth Annual ACM Symposium on Theory of Computing, STOC '78, pages 216-226, New York, NY, USA, 1978. ACM.

[54] Dmitriy Zhuk. A proof of CSP dichotomy conjecture. In 58th IEEE Annual Symposium on Foundations of Computer Science, FOCS 2017, Berkeley, CA, USA, October 15-17, 2017, pages 331-342, 2017.

[55] Dmitriy Zhuk. A proof of the CSP dichotomy conjecture. J. ACM, 67(5), August 2020.

[56] Dmitriy Zhuk. Strong subalgebras and the constraint satisfaction problem. arXiv, abs/2005.00593, 2020.

[57] Dmitriy Zhuk and Barnaby Martin. QCSP monsters and the demise of the Chen conjecture. arXiv, abs/1907.00239, 2019. 


\section{A Preliminaries}

In this section of the appendix we give some additional definitions and list several observations that will be (often implicitly) used in the proofs. In the last subsection we give several characterizations of algebraic concepts by means of relations.

We often abuse the notation and write $t$ both for a term and the induced term operation $t^{\mathbf{A}}$ of an algebra $\mathbf{A}$.

\section{A.1 Star and cyclic composition}

We start with two algebraic concepts, star composition and cyclic composition.

If $t, s$ are operations on the same set of arities $p$ and $q$, then star composition of $t$ and $s$ is defined by

$$
t\left(s\left(x_{1}, x_{p+1}, \ldots, x_{q p-p+1}\right), \ldots, s\left(x_{p}, x_{2 p}, \ldots, x_{q p}\right)\right) .
$$

The star composition of a $p$-ary cyclic operation and a $q$-ary cyclic operation is a cyclic operation of arity $p q$.

If $t$ is a cyclic operation and $s$ is any operation of the same arity $p$, then the cyclic composition of $t$ and $s$ is defined by

$$
t\left(s\left(x_{1}, \ldots, x_{p}\right), s\left(x_{2}, \ldots, x_{p}, x_{1}\right), \ldots, s\left(x_{p}, x_{1}, \ldots, x_{p-1}\right)\right)
$$

and it is a cyclic operation of arity $p$.

\section{A.2 Subpowers and pp-definitions}

Our proofs heavily exploit the fact that subpowers of algebras are closed under pp-definitions.

We have already mentioned in Subsection 2.2 that the relational composition $R+S$, the inverse relation $-R$, and left and right centers of a binary relation $R$ are pp-definable from $\{R, S\}$. Therefore if $R$ and $S$ are subuniverses of $\mathbf{A}^{2}$, then $R+S$ is a subunivere of $\mathbf{A}^{2}$, and $-R$ as well as the centers of $R$ are subuniverses of $\mathbf{A}$ (and this also applies to the multisorted setting when $R$ and $S$ are subuniverses of $\mathbf{A} \times \mathbf{B}$ ). We now discuss further such observations that we use often in this paper.

If $R$ is a subuniverse of $\mathbf{A}^{n}$, then the projection onto a set of coordinates $I \subseteq\{1,2, \ldots, n\}$ is a subpower of $\mathbf{A}$ as well. We sometimes fix some coordinate $i$ to a subuniverse $B \leq \mathbf{A}$ before projecting, i.e., we consider the relation $R^{\prime}\left(x_{1}, \ldots, x_{n}\right) \equiv R\left(x_{1}, \ldots, x_{n}\right) \wedge B\left(x_{i}\right)$.

Let $R$ now be a subdirect subuniverse of $\mathbf{A}^{2}$. Recall that $R$ is linked if $S=(R-R)+\cdots+$ $(R-R)$ is equal to $A^{2}$ when we take a sufficiently large number of summands. In general, $S$ is an equivalence relation on $A$ which is a subuniverse of $\mathbf{A}^{2}$ - a congruence of $\mathbf{A}$. In particular, if $\mathbf{A}$ is simple, then $S$ is either $A^{2}$ (so $R$ is linked) or the equality relation, in which case $R$ is a graph of a permutation $A \rightarrow A$ - an automorphism $\mathbf{A} \rightarrow \mathbf{A}$. So, for simple $\mathbf{A}$, an irredundant $R \leq_{s d} \mathbf{A}^{2}$ is necessarily linked.

One type of subpower is of particular importance. Fix $n$ and consider the subalgebra $\mathbf{F}$ of $\mathbf{A}^{A^{n}}$ with universe $F=\mathrm{Clo}_{n}(\mathbf{A})$, the set of $n$-ary operations on $A$. Thus tuples in $F$ are $n$-ary term operations of $\mathbf{A}$, a coordinate of a tuple in $F$ is an element of $A^{n}$, and the a-th component of a tuple $f \in F$ is the value $f(\mathbf{a})$. The algebra $\mathbf{F}$ is isomorphic to the free algebra for $\mathbf{A}$ over an $n$-element set of generators.

\section{A.3 Absorption and pp-definitions}

Pairs of subpowers of $\mathbf{A}$ with $\mathbf{B} \unlhd \mathbf{C}$ are also closed under pp-definitions in the following sense (this folklore fact was recorded as Lemma 2.9 in [2]).

Lemma A.1. Assume that a subpower $R$ of $\mathbf{A}$ is defined by

$$
R=\left(x_{1}, \ldots, x_{n}\right) \equiv \exists y_{1}, \ldots, y_{m}: R_{1}\left(\sigma_{1}\right) \wedge \cdots \wedge R_{k}\left(\sigma_{k}\right),
$$


where $R_{1}, \ldots, R_{k}$ are subpowers of $\mathbf{A}$ regarded as predicates and $\sigma_{1}, \ldots, \sigma_{k}$ stand for sequences of (free or bound) variables. Let $S_{1}, \ldots, S_{k}$ be subpowers of $\mathbf{A}$ such that $S_{i} \unlhd \mathbf{R}_{i}$ for all $i$. Then the subpower

$$
S\left(x_{1}, \ldots, x_{n}\right) \equiv \exists y_{1}, \ldots, y_{m}: S_{1}\left(\sigma_{1}\right) \wedge \cdots \wedge S_{k}\left(\sigma_{k}\right),
$$

absorbs $\mathbf{R}$. Moreover, if all the absorptions $S_{i} \unlhd \mathbf{R}_{i}$ are witnessed by $t$, then so is $S \unlhd \mathbf{R}$.

In particular, if $R \leq_{s d} \mathbf{A}^{2}$ and $B$ absorbs $\mathbf{A}$, then $B+R$ and $B-R$ absorb $\mathbf{A}$ as well.

It is also useful to observe that absorption is transitive: if $\mathbf{C} \unlhd \mathbf{B}$ by $s$ and $\mathbf{B} \unlhd \mathbf{C}$ by $t$, then $\mathbf{C} \unlhd \mathbf{A}$ by the star composition of $s$ and $t$.

\section{A.4 Relational descriptions}

Many algebraic notions we deal with in this paper have their relational counterparts. We have already stated such a characterization for projectivity in Proposition 3.8 and we have used a relational description of abelianess as a definition for this concept in Definition 3.3 . Now we list three other helpful facts, which are mostly folklore.

The first one is a characterization of absorption by means of so called $B$-essential relations (see e.g. Proposition 2.14 in [2]).

Proposition A.2. Let $\mathbf{A}$ be an algebra and $B \leq \mathbf{A}$. Then $B \unlhd_{n} \mathbf{A}$ if and only if for every $\mathbf{a}^{1}, \ldots, \mathbf{a}^{n} \in A^{n}$ such that $\mathbf{a}_{j}^{i} \in B$ for $i \neq j$ we have $\operatorname{Sg}_{\mathbf{A}^{n}}\left(\mathbf{a}^{1}, \ldots, \mathbf{a}^{n}\right) \cap B^{n} \neq \emptyset$.

The second proposition characterizes strongly projective subuniverses.

Proposition A.3. Let $\mathbf{A}$ be an algebra and $B \subseteq A$. Then $B$ is a strongly projective subuniverse of $\mathbf{A}$ if and only if the relation $R(x, y, z)=B(x) \vee(y=z)$ is a subuniverse of $\mathbf{A}^{3}$.

Proof. For the backward implication let $f$ be an $n$-ary term operation of $\mathbf{A}$ and say, without loss of generality, that the first coordinate is essential as witnessed by tuples $\left(c, c_{2}, \ldots, c_{n}\right)$ and $\left(c^{\prime}, c_{2}, \ldots, c_{n}\right)$. Take $\left(b, a_{2}, \ldots, a_{n}\right) \in B \times A^{n-1}$ and note that $R\left(b, c, c^{\prime}\right), R\left(a_{2}, c_{2}, c_{2}\right), \ldots, R\left(a_{n}, c_{n}\right.$, $\left.c_{n}\right)$. Therefore

$$
R\left(f\left(b, a_{2}, \ldots, a_{n}\right), f\left(c, c_{2}, \ldots, c_{n}\right), f\left(c^{\prime}, c_{2}, \ldots, c_{n}\right)\right)
$$

and, by the choice of $c, c^{\prime}, c_{2}, \ldots, c_{n}$, we get $t\left(b, a_{2}, \ldots, a_{n}\right) \in B$, as required.

For the forward implication we proceed by the way of contradiction and suppose that an application of an operation $f$ to triples from $R$ produces a triple outside. The resulting triple does not have an element of $B$ at the first position, therefore, by the assumption, all the input triples that have an element of $B$ on the first position appear on non-essential coordinates of $f$. The remaining triples have the same element on the second and third positions, therefore so does the resulting triple, a contradiction.

The third proposition shows that strongly functional relations enforce abelianness.

Proposition 4.8. If $R \leq \mathbf{A}^{3}$ is a strongly functional relation, then $\mathbf{A}$ is abelian.

Proof. We define a binary relation $S$ on $A^{2}$ by

$$
\begin{aligned}
& S\left(\left(x_{1}, x_{2}\right),\left(y_{1}, y_{2}\right)\right)=\exists u, v, u^{\prime}, v^{\prime}: \\
& \quad R\left(u, v, x_{1}\right) \wedge R\left(u, v^{\prime}, x_{2}\right) \wedge R\left(u^{\prime}, v, y_{1}\right) \wedge R\left(u^{\prime}, v^{\prime}, y_{2}\right) .
\end{aligned}
$$

Since a tuple of $R$ is determined by any two coordinates, $x_{1}=x_{2}$ implies $v=v^{\prime}$, and this implies $y_{1}=y_{2}$, and vise versa. Since any binary projection of $R$ is full, for any $\left(x_{1}, x_{2}\right)$ we can choose $u$ and then $v, v^{\prime}$ such that $\left(u, v, x_{1}\right),\left(u, v^{\prime}, x_{2}\right) \in R$. Putting $u^{\prime}=u, y_{1}=x_{1}$, and $y_{2}=x_{2}$, we obtain that $S$ is a reflexive relation on $A^{2}$, in particular, the projection of $S$ to any of the two coordinates is $A^{2}$. Finally, any pair of the form $((x, x),(y, y))$ is in $S$ as witnessed by picking $u$ arbitrarily and then choosing $v=v^{\prime}$ and $u^{\prime}$ appropriately. It follows that the "linkedness" congruence $(S-S)+\cdots+(S-S)$ is a congruence on $\mathbf{A}^{2}$ such that one of its blocks is the diagonal $\Delta_{A}$, so $\mathbf{A}$ is abelian. 


\section{B Proofs for Section 4}

This section of the appendix contains proofs of the claims made in Section 4 .

\section{B.1 Absorption theorem}

We start by formally stating the already known improvements and refinements of the proof of the absorption theorem (Theorem 4.1) discussed in Subsection 4.1. Some of these improvements do not seem to be recorded in the literature or are hidden inside proofs of different results. As a corollary we obtain improved an absorption theorem, Corollary 4.5, which seems to be new. Afterwards, we prove the two refinements of the theory which were promised in Subsection 4.1 .

The first step toward Theorem 4.1 is Lemma 2.7. in [7].

Proposition B.1. Every algebra has a transitive operation or a nontrivial projective subuniverse.

The second step has an elementary proof via Taylor operations, see the proof of Lemma 3.4. in [7.

Proposition B.2. Every projective subuniverse of a Taylor algebra is 2-absorbing.

We remark that the argument for Proposition B.2 via cyclic operations sketched in Subsection 4.1 is not "fair" since Theorem 3.2 heavily uses the absorption theorem whose one component is this proposition.

The third step is a purely relational fact and we provide a short proof.

Proposition B.3. Let $R \subseteq s d A \times C$ be proper and linked. Then either $R$ is left central or pp-defines a proper subdirect symmetric central relation on $C$.

Proof. Suppose that $R$ is not left central. Since $R$ (and then $-R$ as well) is linked and subdirect, we have $(-R+R)+(-R+R)+\cdots=C^{2}$ for a sufficiently large number of summands.

If $-R+R \neq C^{2}$, then we first preprocess $R$ by taking $(-R+R)+(-R+R)+\ldots$ sufficiently many times so that $-R+R=C^{2}$ and $R$ is still proper. If it became left central, we are done.

For any set $D=\left\{c_{1}, \ldots, c_{k}\right\} \subseteq C$ consider the binary pp-definable relation $S_{D}(x, y)$ expressing " $x, y$, and all the $c_{i}$ have a common neighbor", that is $S_{D}(x, y) \equiv(\exists a) R(a, x) \wedge R(a, y) \wedge R\left(a, c_{1}\right) \wedge$ $\cdots \wedge R\left(a, c_{k}\right)$. Since $-R+R=C^{2}$, the set $S_{\emptyset}$ is equal to $C^{2}$, and since $R$ is not left central, the set $S_{D}$ is empty. Now take a maximal $D$ so that $S_{D}=C^{2}$, take any $E \subseteq C$ with $|E \backslash D|=1$ and observe that $S_{E}$ is a proper symmetric subdirect relation on $C$ whose center is nonempty (as it contains $E)$.

The fourth step shows how left central relations together with transitive operations on the right side produce absorption. The argument is in the final part of the proof of Theorem 2.11 in 3 .

Proposition B.4. Let $\mathbf{A}$ and $\mathbf{C}$ be algebras and $R \leq_{s d} \mathbf{A} \times \mathbf{C}$. If $\mathbf{C}$ has a transitive term operation, then the left center of $R$ absorbs $\mathbf{A}$.

Proof. Let $t^{\mathbf{C}}$ be a transitive term operation of $\mathbf{C}$, say of arity $n$, and let $B$ be the left center of $R$. We need to show that, for any $\mathbf{a} \in A^{n}$ such that $\left|\left\{i: a_{i} \in B\right\}\right| \geq i-1$, the result $t^{\mathbf{A}}(\mathbf{a})=b$ is in $B$, i.e., we need to show that for every $c \in C$ we have $(b, c) \in R$. Let $i$ be the only coordinate for which $a_{i} \notin B$ (or take $i$ arbitrary if there is none such) and take $d$ such that $\left(a_{i}, d\right) \in R$. By transitivity of $t^{\mathbf{C}}$, there exists $\mathbf{d} \in C^{n}$ with $d_{i}=d$ and $t^{\mathbf{C}}(\mathbf{d})=c$. All the component pairs $\left(a_{j}, b_{j}\right)$ are in $R$ since $B$ is the left center of $R$, so $\left(t^{\mathbf{A}}(\mathbf{a}), t^{\mathbf{C}}(\mathbf{d})\right)=(b, c)$ is in $R$, as required.

Note that the four steps combined already give us the absorption theorem [3] and its special cases formulated as Corollary 4.1 .

Corollary B.5. Suppose $R \leq_{s d} \mathbf{A} \times \mathbf{C}$ is proper and linked, and $\mathbf{C}$ is Taylor. Then $\mathbf{A}$ or $\mathbf{C}$ has a nontrivial absorbing subuniverse. 
Proof. Proposition B.3 gives us left central subuniverse $S$ of either $\mathbf{A} \times \mathbf{C}$ or $\mathbf{C}^{2}$, and for simplicity of presentation assume the former. The left center $B$ of $S$ is a Taylor center unless $\mathbf{C}$ contains a nontrivial proper 2-absorbing subuniverse (in which case we are done). Then $\mathbf{C}$ has no nontrivial projective subuniverses by Proposition B.2 and therefore it has a transitive term operation by Proposition B.1. Proposition B.4 finishes the proof by showing that $B$ is absorbing.

For future reference we also record the following variation.

Corollary B.6. Suppose $R \leq_{s d} \mathbf{A} \times \mathbf{C}$ and $\mathbf{C}$ is Taylor. Then $\mathbf{C}$ has a nontrivial projective and 2-absorbing subuniverse, or the left center of $R$ absorbs $\mathbf{A}$.

Proof. Either $\mathbf{C}$ has a nontrivial projective subuniverse (which is 2-absorbing by Proposition B.2) or $\mathbf{C}$ has a transitive term operation by Proposition B.1 and then the left center absorbs $\mathbf{A}$ by Proposition B.4.

The fifth step is to improve absorption to 3-absorption. We further divide this task to two sub-steps. The first one isolates a property of centers that was, inspired by this paper, exploited in 56 .

Lemma B.7. Let $B$ be a center of $\mathbf{A}$ and let $a \in A \backslash B$. Then $(a, a)$ is not in the subuniverse of $\mathbf{A}^{2}$ generated by $(\{a\} \times B) \cup(B \times B) \cup(B \times\{a\})$.

Proof. Let $R \leq_{s d} \mathbf{A} \times \mathbf{C}$ be a witness of centrality. Suppose, for a contradiction that $(a, a)$ is generated by a term operation $f^{\mathbf{A}}$, so

$$
f^{\mathbf{A}}\left(a, \ldots, a, b_{1}, \ldots, b_{i}\right)=a=f^{\mathbf{A}}\left(b_{1}^{\prime}, \ldots, b_{j}^{\prime}, a, \ldots, a\right)
$$

for some $b_{1}, \ldots, b_{i}, b_{1}^{\prime}, \ldots, b_{j}^{\prime} \in B$ where $i+j$ is not less than the arity of $t$. Therefore $f^{\mathbf{C}}(a+$ $\left.R, \ldots, a+R, b_{1}+R, \ldots, b_{i}+R\right) \subseteq a+R$ and, denoting $D=a+R$, we have $f^{\mathbf{C}}(D, \ldots, D, C, \ldots, C) \subseteq$ $D$ (with $i$ occurrences of $C$ on the left). Similarly, we obtain $f^{\mathbf{C}}(C, \ldots, C, D, \ldots, D) \subseteq D$ with $j$ occurrences of $C$. It follows that the binary operation on $C$ obtained from $f^{\mathbf{C}}$ by identifying the first $j$ variables to $x$ and the rest to $y$ witnesses the nontrivial binary absorption $D \unlhd_{2} \mathbf{C}$, a contradiction with the definition of a center.

The second sub-step derives 3-absorption from absorption and a weakening of the property of centers isolated in the first sub-step.

Proposition B.8. Suppose $B \unlhd \mathbf{A}$ and that for every $a \in A \backslash B$ we have $(a, a) \notin \operatorname{Sg}_{\mathbf{A}^{2}}(\{a\} \times B \cup$ $B \times\{a\})$. Then $B$ 3-absorbs $\mathbf{A}$.

Proof. Let $n$ be the minimal number such that $B \unlhd_{n+1} \mathbf{A}$ and assume, striving for a contradiction, that $n>2$. By Proposition $\$$.2 there exist $\mathbf{a}^{1}, \ldots, \mathbf{a}^{n} \in A^{n}$ such that $a_{j}^{i} \in B$ for $i \neq j$ and $\operatorname{Sg}_{\mathbf{A}^{n}}\left(\mathbf{a}^{1}, \ldots, \mathbf{a}^{n}\right) \cap B^{n}=\emptyset$. Put $R=\operatorname{Sg}_{\mathbf{A}^{n}}\left(\mathbf{a}^{1}, \ldots, \mathbf{a}^{n}\right)$ and assume that $R$ is an inclusion minimal relation among all choices of $\mathbf{a}^{1}, \ldots, \mathbf{a}^{n} \in A^{n}$.

By $S$ we denote the binary relation defined by

$$
\operatorname{Sg}_{\mathbf{A}^{2}}\left(\left(\left\{a_{n}^{n}\right\} \times B\right) \cup\left(B \times\left\{a_{n}^{n}\right\}\right)\right) .
$$

and we pp-define $R^{\prime} \leq \mathbf{A}^{2 n-2}$ by the formula

$$
\begin{aligned}
& R^{\prime}\left(x_{1}, \ldots, x_{n-1}, x_{1}^{\prime}, \ldots, x_{n-1}^{\prime}\right)=\exists x_{n}, x_{n}^{\prime}: \\
& R\left(x_{1}, \ldots, x_{n}\right) \wedge R\left(x_{1}^{\prime}, \ldots, x_{n}^{\prime}\right) \wedge S\left(x_{n}, x_{n}^{\prime}\right) .
\end{aligned}
$$

For $i \in\{1, \ldots, n\}$ by $\mathbf{c}^{i}$ we denote $\mathbf{a}^{i}$ take away the last coordinate. By the definition of $R^{\prime}$ and $S$, we have $\left(\mathbf{c}^{i}, \mathbf{c}^{n}\right),\left(\mathbf{c}^{n}, \mathbf{c}^{i}\right) \in R^{\prime}$ for every $i \in\{1, \ldots, n-1\}$. Moreover, these $2 n-2$ tuples satisfy the condition in the second part of Proposition A.2. Therefore, if $R^{\prime} \cap B^{2 n-2}=\emptyset$, then $B \unlhd_{2 n-2}$ A. But $B \unlhd_{n+1} \mathbf{A}$ and $2 n-2 \geq n+1$, a contradiction. 
It now remains to show that $R^{\prime} \cap B^{2 n-2}=\emptyset$. Assuming the converse, there exist $\mathbf{d}, \mathbf{d}^{\prime} \in$ $R \cap\left(B^{n-1} \times A\right)$ such that $\left(d_{n}, d_{n}^{\prime}\right) \in S$. Let $E$ be the projection of $R$ onto the last coordinate after fixing all the other coordinates to $B$, that is, $E=\operatorname{proj}_{n}\left(R \cap\left(B^{n-1} \times A\right)\right)$. Since $R$ was chosen inclusion minimal, we get $\operatorname{proj}_{n}(R)=\operatorname{Sg}_{\mathbf{A}}(B \cup\{e\})$ for every $e \in E$, otherwise we could replace $\mathbf{a}^{n}$ by a tuple $\mathbf{b} \in R \cap\left(B^{n-1} \times\{e\}\right)$ and get a $B$-essential relation properly contained in $R$.

Let $E^{\prime}=E+S$. Since $B \cup\left\{d_{n}, d_{n}^{\prime}\right\} \subseteq E^{\prime}$ (as $E$ contains $a_{n}^{n}, d_{n}, d_{n}^{\prime}$ and $\left(d_{n}, d_{n}^{\prime}\right) \in S$ ), we have $E^{\prime}=\operatorname{proj}_{n}(R)$, in particular $a_{n}^{n} \in E^{\prime}$. Hence, for $E^{\prime \prime}=a_{n}^{n}+S$ we have $B \subseteq E^{\prime \prime}$ and $E^{\prime \prime} \cap E \neq \emptyset$. Therefore, $a_{n}^{n} \in E^{\prime \prime}$ and $\left(a_{n}^{n}, a_{n}^{n}\right) \in S$. This directly contradicts our assumption on $B$.

The improved absorption theorem, and its special case formulated as Corollary 4.5, is now a straightforward consequence.

Corollary B.9. Suppose $R \leq_{s d} \mathbf{A} \times \mathbf{C}$ is proper and linked, and $\mathbf{C}$ is Taylor. Then $\mathbf{A}$ or $\mathbf{C}$ has a nontrivial 3-absorbing subuniverse.

Proof. The proof goes the same way as in Corollary B.5 until the end when we additionally apply Lemma B.7 and Proposition B.8.

Proposition 4.3, which is Corollary 7.10.2 in [55], is also an immediate consequence.

Proposition 4.3. [55] If $B$ is a Taylor center of an algebra $\mathbf{A}$, then $B \unlhd_{3} \mathbf{A}$.

Proof. The proof is obtained by skipping the first two sentences in the proof of Corollary B.5 and in the end applying Lemma B.7 and Proposition B.8

Now we move on to the improvements of the theory. The first one is that centers of Taylor algebras absorb (as opposed to Taylor centers in arbitrary algebras as in Proposition 4.3).

Proposition 4.4. If $B$ is a center of a Taylor algebra $\mathbf{A}$, then $B \unlhd_{3} \mathbf{A}$.

Proof. Let $R \leq_{s d} \mathbf{A} \times \mathbf{C}$ be the witnessing relation from the definition of center. We define a directed graph on $A$, by putting $c \rightarrow d$ if there exists a cyclic term $t$ and a choice of elements $b_{2}, \ldots, b_{n} \in B$ such that $t^{\mathbf{A}}\left(c, b_{2}, \ldots, b_{n}\right)=d$.

Obviously the graph has no sinks, but it also easy to see that it is transitive. Indeed, let $c \rightarrow c^{\prime}$ by $t$ and $b_{2}, \ldots, b_{n}$ and $c^{\prime} \rightarrow c^{\prime \prime}$ by $t^{\prime}$ and $b_{2}^{\prime}, \ldots, b_{n^{\prime}}^{\prime}$. Then the star composition of $t^{\prime}$ and $t$, together with the tuple

$$
b_{2}, \ldots, b_{n}, \underbrace{b_{2}^{\prime}, \ldots, b_{2}^{\prime}}_{n}, \ldots, \underbrace{b_{n^{\prime}}, \ldots, b_{n^{\prime}}}_{n},
$$

reordered as in the definition of star composition, witnesses the edge $c \rightarrow c^{\prime \prime}$.

Next we claim that there is no $a \in A \backslash B$ with a self-loop. For a contradiction, say $a$ is such and let $t^{\mathbf{A}}\left(a, b_{2}, \ldots, b_{n}\right)=a$ with $a \in A \backslash B$ and $b_{2}, \ldots, b_{n} \in B$. The set $D=\{a\}+R$ is a subuniverse of $\mathbf{C}$, and moreover

$$
t^{\mathbf{C}}\left(\{a\}+R,\left\{b_{2}\right\}+R, \ldots,\left\{b_{n}\right\}+R\right)=t^{\mathbf{C}}(D, C, \ldots, C) \subseteq D
$$

and the same holds for all the cyclic shifts of $t$. Therefore $D$ is 2-absorbing subuniverse $\mathbf{C}$ (by the operation $\left.t^{\mathbf{C}}(x, y, \ldots, y)\right)$, a contradiction.

We conclude that all the loops and directed cycles in this graph must be inside $B$. Therefore there is a number $m$ so that starting with any $a \in A$, and following any directed walk, after $m$ steps we necessarily arrive in $B$. It suffices to take any cyclic term of $\mathbf{A}$ and star-compose it with itself $m$ times to obtain an operation which witnesses $\mathbf{B} \unlhd \mathbf{A}$. Now it is enough to apply Lemma B.7 and Proposition B.8

The second improvement, Proposition 4.2, shows that every linked relation $R \subseteq A \times B$ with $A=B$ pp-defines a central relation which is additionally symmetric or transitive.

Proposition 4.2. Let $R \subseteq s d A^{2}$ be linked and proper. Then $R$ pp-defines a subdirect proper central relation on $A$ which is symmetric or transitive. 
Proof. If left or right center of $R$ is empty we apply Proposition B.3 to $R$ itself, or to $-R$, and the result follows. So, let $R$ be central. We also assume, without loss of generality, that the left center of $R$ contains the maximal number of elements among central, proper and subdirect relations pp-definable from $R$.

Consider the sequence of subdirect relations $R_{0}=R, R_{i+1}=R_{i}+R_{i}$. As is easily seen by induction, every $R_{i}$ is subdirect and central. Indeed, $R_{0}=R$ satisfies these conditions, suppose so does $R_{i}$. Then for any $a \in A$ there is $b, c \in A$ such that $(a, b),(b, c) \in R_{i}$, implying $(a, c) \in R_{i+1}$ and $\operatorname{proj}_{1} R_{i+1}=A$. The equality $\operatorname{proj}_{2} R_{i+1}=A$ is similar. If $a$ belongs to the left center of $R_{i}$, i.e. $(a, b) \in R_{i}$ for each $b \in A$, then, in particular, $(a, a) \in R_{i}$. Therefore $(a, b) \in R_{i+1}$ for all $b \in A$, and $a$ belongs to the left center of $R_{i+1}$. A similar argument shows that the right center of $R$ is a subset of the right center of each $R_{i}$. Note that this implies that the left centers of all the proper relations $R_{i}$ are equal: they all contain the left center of $R$, and, by the choice of $R$ to have a largest left center, they have to be equal to that of $R$.

For some $N$ it holds that $R_{N}=R_{N+1}$, choose the smallest number with this property. If $R_{N} \neq A^{2}$ then it is the desired proper central and transitive relation on $A$. If $R_{N}=A^{2}$, consider $S=R_{N-1}$. It is a subdirect, proper central relation such that $S+S=A^{2}$.

Next, let $B$ be the right center of $S$, we consider two cases: either $B+S=A$ or $B+S \neq A$. (Note that $B$ is the right center, which implies $B-S=A$, but not necessarily $B+S=A$. So the latter case is possible.)

CASE $1 . B+S=A$.

Consider $S^{\prime}=S \cap-S$. This relation is proper, because $S$ is proper and is symmetric by construction. It is also subdirect, as $S+S=A^{2}$ implies that for every $a$ there is $b$ such that $S(a, b)$ and $S(b, a)$. Finally, $S^{\prime}$ is also linked. Indeed, note that, since $B$ is the right center, $B^{2} \subseteq S$, and so $B^{2} \subseteq S^{\prime}$. Also, the assumption $B+S=A$ implies that for any $a \in A$ there is $b \in B$ such that $(b, a) \in S$. On the other hand, $(a, b) \in S$, because $b$ belongs to the right center. Therefore $(a, b) \in S^{\prime}$, implying together with $B^{2} \subseteq S^{\prime}$ that $S^{\prime}$ is linked.

If $S^{\prime}$ is central, then we are done. Otherwise, since $S^{\prime}$ is symmetric, its right center is empty, and we use Proposition B.3 to obtain a symmetric central relation.

CAse 2. $B+S \neq A$.

We will derive a contradiction that shows that this case is impossible. Let $A=\left\{a_{1}, \ldots, a_{n}\right\}$, and for $j \geq 0$, let the relation $T_{j}$ be given by

$$
T_{j}(x, y)=(\exists z) S(x, z) \wedge S(z, y) \wedge \bigwedge_{i=1}^{j} S\left(a_{i}, z\right) .
$$

Clearly, $T_{0}=A^{2}$ and $T_{n}$ is not even subdirect. Indeed, for the latter claim if $(a, b) \in T_{n}$, then the value of $z$ in the pp-definition above belongs to $B$, the right center. As $B+S \neq A$, there is $c \in A$ such that $(z, c) \notin S$ for any feasible choice of $z$, witnessing that $c \notin \operatorname{proj}_{2} T_{n}$. Therefore there is $j$ such that $T_{j-1}=A^{2}$, and $T_{j} \neq A^{2}$. We will show that $T_{j}$ is central and has strictly larger left center than $S$, which contradicts the choice of $R$.

By the definition of $T_{j}$ we have $\left(a_{j}, b\right) \in T_{j-1}$ if and only if $T_{j}\left(a_{j}, b\right)$, therefore $\left\{a_{j}\right\}+T_{j}=A$. This implies that $\operatorname{proj}_{2}\left(T_{j}\right)=A$ and that $a_{j}$ is in the left center of $T_{j}$. Note that every element in the left center of $S$ is in the left center of $T_{j}$. Indeed, if $a$ is in the left center of $S$, then it is also in the right center of $S$. Therefore for any choice of $b$, the value of $z$ can be set to $a$, proving that $(a, b) \in T_{j}$. Note also that $a_{j}$ does not belong to the left center of $S$, because this would imply that $T_{j-1}=T_{j}$. Since $S$ has non-empty right center, for any $a \in A$, choose the value of $z$ in the definition of $T_{j}$ to be from the right center. Then $(a, z),\left(a_{1}, z\right), \ldots,\left(a_{j}, z\right) \in S$, and a value for $y$ can be chosen with $(z, y) \in S$, implying that $\operatorname{proj}_{1}\left(T_{j}\right)=A$.

Thus $T_{j}$ is proper, subdirect, central and pp-definable from $R$. However, its left center is a superset of the left center of $R$ and contains $a_{j}$ that is not in the left center of $R$. A contradiction with the choice of $R$.

The proposition is proved. 


\section{B.2 Subdirect irredundant subpowers}

In this subsection we prove our main pp-definability result, Theorem 4.7, and Theorems 4.10 and 4.11 that will give us enough edges for the connectivity theorem. We start with a technical lemma.

Lemma B.10. Let $R \subseteq$ sd $A^{n}$ be a relation, and $I \subseteq n$ be a maximal set of coordinates such that $\operatorname{proj}_{I}(R)$ is the full product $A^{|I|}$. Then every tuple in $R$ is determined by its projection to $I$, or $R$ pp-defines $R^{\prime} \subseteq$ sd $A^{2}$ which is irredundant and proper,

Proof. Let $R$ be a counterexample minimal with respect to the arity. In particular, no relation ppdefinable from $R$, such as the projection to a subset of variables, pp-defines subdirect, irredundant, and proper binary relation.

The relation $R$ needs to be irredundant, otherwise the projection forgetting one of the redundant coordinates is a counterexample to the lemma of smaller arity. Next, $|I|$ has to be $n-1$, because otherwise the lemma holds for every projection $S$ of $R$ on any $|I|+1$ coordinates containing $I$, let $j$ be the additional coordinate position. Relation $S$ does not pp-define a subdirect, irredundant and proper binary relation, so by the minimality of $I$ for any $\mathbf{b} \in S$ the value $a_{j}$ is determined by the remaining coordinates, and that also holds for $R$. Without loss of generality assume $I=\{1, \ldots, n-1\}$. Consider $\operatorname{proj}_{1, \ldots, n-2, n} R$. By the minimality of $R$ it has to be full. Indeed, otherwise the first $n-2$ coordinates determine the $n$-th coordinate, contradicting the assumption that $R$ is a minimal counterexample.

Since $R$ is a counterexample there are elements $a \neq a^{\prime}$ and tuples $\left(a_{1}, \ldots, a_{n-1}, a\right),\left(a_{1}, \ldots\right.$, $\left.a_{n-1}, a^{\prime}\right) \in R$. Also, as $R$ is not full, $\left(c_{1}, \ldots, c_{n}\right) \notin R$ for some tuple. Set $T=\left\{\left(b_{1}, \ldots, b_{n-1}\right)\right.$ : $\left.\left(b_{1}, \ldots, b_{n-1}, c_{n}\right) \in R\right\}$; we show that either $T$ or $\operatorname{proj}_{1, \ldots, n-2, n} R$ is a smaller counterexample, thus obtaining a contradiction. Indeed, the relation $T$ is proper, as it does not contain $\left(c_{1}, \ldots, c_{n-1}\right)$. Also, $\operatorname{proj}_{1, \ldots, n-2} T$ is the full relation, because otherwise $\operatorname{proj}_{1, \ldots, n-2, n} R$ would be a proper relation, and therefore a smaller counterexample. It remains to show that the values of the first $n-2$ coordinate positions of $T$ do not determine the last one. To this end we consider an auxiliary binary relation $S$ given by $S=\left\{(a, b):\left(a_{1}, \ldots, a_{n-2}, a, b\right) \in R\right\}$. This relation is subdirect, as both $\operatorname{proj}_{I} R$ and $\operatorname{proj}_{1, \ldots, n-2, n} R$ are full relations. Relation $S$ is also irredundant, because $\left(a_{n-1}, a\right),\left(a_{n-1}, a^{\prime}\right) \in S$. By the assumptions about $R$, relation $S$ cannot be proper. Therefore the tuples $\left(a_{1}, \ldots, a_{n-2}, a, c_{n}\right),\left(a_{1}, \ldots, a_{n-2}, a^{\prime}, c_{n}\right) \in R$, implying that $\left(a_{1}, \ldots, a_{n-2}, a\right),\left(a_{1}, \ldots, a_{n-2}, a^{\prime}\right) \in T$.

We are now in a position to prove Theorem 4.7.

Theorem 4.7. Let $R \subseteq_{s d} A^{n}$ be an irredundant proper relation. Then either

- $R$ pp-defines $R^{\prime} \subseteq$ sd $A^{2}$ which is irredundant and proper, or

- there exist ternary relations $R_{1}, \ldots, R_{n} \subseteq$ sd $A^{3}$ such that each $R_{i}$ is strongly functional and the set $\left\{R_{1}, \ldots, R_{m}\right\}$ is inter-pp-definable with $R$.

Proof. First, we argue that $R$ pp-defines some binary or ternary proper and irreducible relations. Let $R^{\prime}$ be a proper irreducible relation of minimal arity pp-definable by $R$. If $R^{\prime}$ is binary or ternary, we are done. Otherwise observe that the projection of $R^{\prime}$ on any proper set of coordinates is the full relation. Let $\left(a_{1}, \ldots, a_{k}\right) \notin R^{\prime}$. Consider the relation $S$ given by

$$
S=\left\{\left(x_{1}, \ldots, x_{k}-1\right):\left(x_{1}, \ldots, x_{k-1}, x_{k-1}, a_{k}\right) \in R^{\prime}\right\} .
$$

This relation is proper, as $\left(a_{1}, \ldots, a_{k}\right) \notin R^{\prime}$. It is also subdirect, because every binary projection of $R^{\prime}$ is the full relation. If $S$ is redundant, say, for $i, j \in\{1, \ldots, k-1\}$ it holds that $\operatorname{proj}_{i j} S$ is the graph of bijection, then $\operatorname{proj}_{i j k} R^{\prime}$ is a proper relation, a contradiction with the choice of $R^{\prime}$.

If $R$ pp-defines a binary, proper, and irreducible relation, the first item from the conclusion of the theorem holds. So, suppose that such a binary relation cannot be defined. Let $R_{1}, \ldots, R_{m} \subseteq$ sd $A^{3}$ be all the proper, ternary, irredundant and subdirect relations in the clone. Any binary projection of each $R_{i}$ is the full relation, and by Lemma B.10, any tuple from $R_{i}$ is determined by any of its two entries. It remains to prove that the set $\left\{R_{1}, \ldots, R_{m}\right\}$ pp-defines $R$. 
We show, by induction on the arity, that any at least ternary irredundant subdirect relation ppdefinable from $R_{1}, \ldots, R_{m}, R$ is also pp-definable from $R_{1}, \ldots, R_{m}$. We proceed by contradiction, let $S \subseteq s d A^{l}$ be a counterexample of minimal arity, that is, $R_{1}, \ldots, R_{m}, R$ pp-define $S$, but $R_{1}, \ldots, R_{m}$ do not. By Lemma B.10 there is $I \subseteq\{1, \ldots, l\}$, such that any tuple $\left(a_{1}, \ldots, a_{l}\right) \in S$ is determined by its projection on $I$ and $\operatorname{proj}_{I} S$ is the full relation. Assume $I=\{1, \ldots, k\}$. If $k+1 \neq l$, then

$$
S\left(x_{1}, \ldots, x_{k}\right)=\bigwedge_{j=k+1}^{l} \operatorname{proj}_{I \cup\{j\}} S\left(x_{1}, \ldots, x_{k}, x_{j}\right) .
$$

By the induction hypothesis every $\operatorname{proj}_{I \cup\{j\}} S$ is pp-definable from $R_{1}, \ldots, R_{m}$, hence, so is $S$, a contradiction with the choice of $S$. Thus we may assume that $k+1=l$.

Claim. Each projection $S^{\prime}=\operatorname{proj}_{J} S$ on $J \subseteq\{1, \ldots, l\}$ with $|J|<l$ is full.

Indeed, it is the case if $J \subseteq I$ by the choice of $I$. Therefore $l \in J$, and, as $\operatorname{proj}_{J \backslash\{l\}} S^{\prime}$ is the full relation, by Lemma B.10 every tuple from $S^{\prime}$ is determined by its projection to $J \backslash\{l\}$. This means that $S$ can obtained from $S^{\prime}$ by extending the tuples from $S^{\prime}$ in an arbitrary way, and this a pp-definition of $S$ from $S^{\prime}$. By induction hypothesis $S^{\prime}$ is pp-definable from $R_{1}, \ldots, R_{m}$, and we obtain a contradiction with the choice of $S$.

Since $S$ is proper, there is $\left(c_{1}, \ldots, c_{l}\right) \notin S$. Set $T=\left\{(a, b, c):\left(c_{1}, \ldots, c_{l-3}, a, b, c\right) \in S\right\}$. This relation is proper, since $\left(c_{l-2}, c_{l-1}, c_{l}\right) \notin T$. By the Claim above $\operatorname{proj}_{1,2}(T)=\operatorname{proj}_{1,3}(T)=$ $\operatorname{proj}_{2,3}(T)=A^{2}$. Thus, $T$ is one of the $R_{i}$ 's. Consider relations $U$ and $U^{\prime}$ given by

$$
U\left(x_{1}, \ldots, x_{l}, y\right)=S\left(x_{1}, \ldots, x_{l}\right) \wedge T\left(y, x_{l-1}, x_{l}\right) .
$$

and

$$
\begin{aligned}
U^{\prime}\left(x_{1}, \ldots, x_{l}, y\right)= & \operatorname{proj}_{1, \ldots, l-2, l+1} U\left(x_{1}, \ldots, x_{l-2}, y\right) \\
& \wedge T\left(y, x_{l-1}, x_{l}\right)
\end{aligned}
$$

We show that that they are identical. This will imply the result, because, as is easily seen, $S=\operatorname{proj}_{1, \ldots, l} U$, and $U^{\prime}$ is pp-definable from $R_{1}, \ldots, R_{m}$, as $\operatorname{proj}_{1, \ldots, l-2, l+1}(U)$ is by the induction hypothesis.

It is not hard to see that $U^{\prime} \subseteq U$. Next, note that $U^{\prime \prime}=\operatorname{proj}_{1, \ldots, l-2, l+1} U$ is not full, since $U\left(c_{1}, \ldots, c_{l-3}, a, b, c, d\right)$ imply that $d=a$. On the other hand, $\operatorname{proj}_{1, \ldots, l-2} U$ is full. Therefore by Lemma B.10 for any $\left(a_{1}, \ldots, a_{l}, a\right) \in U$ the value $a$ is determined by $a_{1}, \ldots, a_{l-2}$.

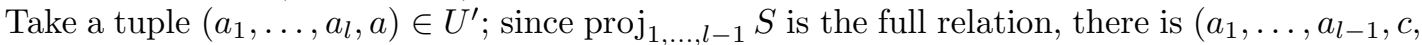
$d) \in U$ for some $c, d \in A$. Since $\left(a_{1}, \ldots, a_{l-2}, a\right) \in \operatorname{proj}_{1, \ldots, l-2, l+1} U$, and in this relation the last value is determined by the first $l-2$ ones, we have $d=a$. Again by Lemma B.10 the third coordinate of the relation $T$ is determined by the first two ones. Therefore, as we have $T\left(d, a_{l-i}, c\right)$ from the definition of $U, T\left(a, a_{l-i}, a_{l}\right)$ from the definition of $U^{\prime}$, and $d=a$, we also obtain $c=a_{l}$. Thus, $\left(a_{1}, \ldots, a_{l}, a\right) \in U$ completing the proof.

As already discussed in Section 4, Theorem 4.7 implies that every algebra $\mathbf{A}$ has at least one of the following properties of its invariant relations.

(1) A has no proper irredundant subdirect subpowers.

(2) A has a proper irredundant binary subdirect subpower.

(3) A has a ternary strongly functional subpower.

Proposition 4.8 shows that $\mathbf{A}$ is abelian in case (2). The next theorem produces majority edges in case (1) when $\mathbf{A}$ is simple.

Theorem 4.10. Suppose that A is simple and has no subdirect proper irredundant subpowers. Then there exists a term operation $t \in \mathrm{Clo}_{3}(\mathbf{A})$ such that for any $(a, b) \notin \mu_{\mathbf{A}}, t(a, a, b)=t(a, b, a)=$ $t(b, a, a)=a$. 
Proof. We assume that $\mu_{\mathbf{A}}$ is not full as otherwise the claim is void. We consider the subalgebra $\mathbf{F}$ of $\mathbf{A}^{A^{3}}$ formed by all the ternary term operations of $\mathbf{A}$ and let $\mathbf{S} \leq \mathbf{A}^{I}$ be the projection of $\mathbf{F}$ onto the set of coordinates $I=\left\{\left(a_{1}, a_{2}, a_{3}\right): \exists a, b\left\{a_{1}, a_{2}, a_{3}\right\}=\{a, b\} \wedge \neg \mu_{\mathbf{A}}(a, b)\right\}$.

The subpower $S$ can be redundant; suppose, e.g., that $\operatorname{proj}_{\left(a_{1}, a_{2}, a_{3}\right),\left(a_{1}^{\prime}, a_{2}^{\prime}, a_{3}^{\prime}\right)} S$ is a graph of a bijection. Note that, in this case, the position of a non-repeating element in $\left(a_{1}, a_{2}, a_{3}\right)$ and in $\left(a_{1}^{\prime}, a_{2}^{\prime}, a_{3}^{\prime}\right)$ must be the same and we can assume, without loss of generality, that $\left(a_{1}, a_{2}, a_{3}\right)=$ $(a, b, b)$ and $\left(a_{1}^{\prime}, a_{2}^{\prime}, a_{3}^{\prime}\right)=\left(a^{\prime}, b^{\prime}, b^{\prime}\right)$. The fact that $\operatorname{proj}_{(a, b, b),\left(a^{\prime}, b^{\prime}, b^{\prime}\right)} S$ is a graph of bijection implies that, for every term operation $t$, if $t(a, b, b)=t(b, a, b)=t(b, b, a)=b$ then $t\left(a^{\prime}, b^{\prime}, b^{\prime}\right)=$ $t\left(b^{\prime}, a^{\prime}, b^{\prime}\right)=t\left(b^{\prime}, b^{\prime}, a^{\prime}\right)=b^{\prime}$. This means that we can consider $S^{1}=\operatorname{proj}_{J}(S)$ where $J=I \backslash$ $\left\{\left(a^{\prime}, b^{\prime}, b^{\prime}\right),\left(b^{\prime}, a^{\prime}, b^{\prime}\right),\left(b^{\prime}, b^{\prime}, a^{\prime}\right)\right\}$ instead of $S$. We continue removing redundant coordinates in this way to arrive at a subpower $S^{i}$ which is irredundant and therefore, by our assumption, full. In particular, we obtain a term operation acting as the majority on the evaluations corresponding to coordinates remaining in $S^{i}$ and, by construction, also on all other evaluations required to prove the theorem.

In case (2) and when $\mathbf{A}$ is simple we get semilattice edges by the following theorem.

Theorem 4.11. Suppose $\mathbf{A}$ with $|A|>2$ is simple and there exists a proper irredundant subdirect binary subpower. Then there exists $\mu_{\mathbf{A}}$-class $B$ such that, for every $b \in B, a \notin B$, the pair $(a, b)$ is a semilattice edge witnessed by the identity congruence.

Proof. We prove the conclusion in a number of claims. We start with a basic observation: if $R \leq_{s d} \mathbf{A}^{2}$ and $(a, b),\left(a^{\prime}, b\right)$ for $\left(a, a^{\prime}\right) \notin \mu_{\mathbf{A}}$ then $b$ is in the right center of $R\left(\right.$ since $\left.\operatorname{Sg}_{\mathbf{A}}\left(a, a^{\prime}\right)=A\right)$.

Claim 1. Every irredundant, proper $R \leq_{s d} \mathbf{A}^{2}$ is central.

Proof. Since $\mathbf{A}$ is simple, $R$ needs to be linked. Therefore, since $\mu_{\mathbf{A}}$ is not the full relation, we have $(a, b),\left(a^{\prime}, b\right) \in R$ for $\left(a, a^{\prime}\right) \notin \mu_{\mathbf{A}}$ which implies that $b$ is in the right center of $R$. The proof for left center is symmetric.

Claim 2. Let $R \leq_{s d} \mathbf{A}^{2}$ be irredundant and proper. If a and $a^{\prime}$ are in the left (right) center of $R$ then $\left(a, a^{\prime}\right) \in \mu_{\mathbf{A}}$.

Proof. Suppose not. Then for every $b \in A$ we have $(a, b),\left(a^{\prime}, b\right) \in R$ which implies that $b$ is the right center. This cannot happen in a proper $R$.

Claim 3. Let $R \leq_{s d} \mathbf{A}^{2}$ be irredundant and proper. If $a$ is in the left center of $R$ and $a^{\prime}$ in the right center then $\left(a, a^{\prime}\right) \in \mu_{\mathbf{A}}$.

Proof. Suppose, for a contradiction, that $a$ is in the left center of $R, a^{\prime}$ in the right center and $\left(a, a^{\prime}\right) \notin \mu_{\mathbf{A}}$. Since $(a, a),\left(a^{\prime}, a^{\prime}\right) \in R$ then, for every $b \in A$, we have $(b, b) \in R$.

Take $a^{\prime \prime}$ such that $\left(a^{\prime \prime}, a^{\prime}\right) \notin \mu_{\mathbf{A}}$ then $a^{\prime \prime}$ is in the left center of $R$, as both $\left(a^{\prime \prime}, a^{\prime \prime}\right)$ and $\left(a^{\prime \prime}, a^{\prime}\right)$ are in $R$. This implies, by the previous claim, that $\left(a^{\prime \prime}, a\right) \in \mu_{\mathbf{A}}$.

In particular we conclude that $\mu_{\mathbf{A}}$ has two equivalence blocks, and that every $a^{\prime \prime}$ such that $\left(a, a^{\prime \prime}\right) \in \mu_{\mathbf{A}}$ is in the left center. By symmetry we get $R=\left(a / \mu_{\mathbf{A}} \times A\right) \cup\left(A \times a^{\prime} / \mu_{\mathbf{A}}\right)$, but then $R \cap-R$ is a proper congruence (recall $|A|>2$ ) on a simple algebra $\mathbf{A}$, a contradiction.

Claim 4. Let $R \leq_{s d} \mathbf{A}^{2}$ be irredundant and proper. There is an $\mu_{\mathbf{A}}$ block $B$ such that $R \subseteq$ $B \times A \cup A \times B$.

Proof. Fix $a$ in the left center of $R$ and $a^{\prime}$ in the right; by the previous claim $\left(a, a^{\prime}\right) \in \mu_{\mathbf{A}}$. We will show, that if $(b, c) \in R$ then $(b, a) \in \mu_{\mathbf{A}}$ or $\left(c, a^{\prime}\right) \in \mu_{\mathbf{A}}$, which proves the claim with $B=a / \mu_{\mathbf{A}}=a^{\prime} / \mu_{\mathbf{A}}$. Indeed, if $(b, a) \notin \mu_{\mathbf{A}}$ then, as $(b, c),(a, c) \in R$ and $c$ is in the right center of $R$ and thus $\left(c, a^{\prime}\right) \in \mu_{\mathbf{A}}$.

Claim 5. Let $R \leq_{s d} \mathbf{A}^{2}$ be irredundant and proper and $S \leq_{s d} \mathbf{A}^{2}$ redundant. If $B$ is the block defined by the previous claim for $R$, then $B+S=B$. 
Proof. Suppose not and let $\left(a, a^{\prime}\right) \in S$ with $a \in B$ and $a^{\prime} \notin B$. Then $a$ is in the left center of $R+S$ while $a^{\prime}$ in the right center of $R+S$ contradicting Claim 3 (the relation $R+S$ is clearly irredundant and proper).

Claim 6. Let $R, S \leq_{s d} \mathbf{A}^{2}$ be irredundant and proper. If $B, C$ are the blocks defined by the Claim 4 for $R, S$ respectively, then $B=C$.

Proof. Suppose, for a contradiction, that $B \neq C$. Let $b, b^{\prime}$ be the elements of the right and left centers of $R$, respectively, and similarly $c, c^{\prime}$ for $S$. Let $T=R \cap S$, and note that $\left(b, c^{\prime}\right),\left(c, b^{\prime}\right) \in T$. As $(b, c) \notin \mu_{\mathbf{A}}$ and $\left(c^{\prime}, b^{\prime}\right) \notin \mu_{\mathbf{A}}$, the relation $T$ is subdirect in $A^{2}$.

Since $B \cap C=\emptyset$ the relation $T$ has no center, and thus by Claim 1 needs to be redundant. But then $(B+T) \cap C \neq \emptyset$ which contradicts the previous claim.

To finish the proof we take a proper irredundant subdirect binary relation provided by the assumption and set $A^{\prime}$ to be the block defined for it by Claim 4 .

Take any $a^{\prime} \in A^{\prime}, b \notin A^{\prime}$ and let $R=\operatorname{Sg}_{\mathbf{A}^{2}}\left(\left(a^{\prime}, b\right),\left(b, a^{\prime}\right)\right)$. The relation $R$ cannot be redundant as $b \in A^{\prime}+R$ would contradict Claim [5. Thus, by Claim 1 and Claim 6, there is $a^{\prime \prime} \in A^{\prime}$ in the left center of $R$. Since $\left(b, a^{\prime}\right),\left(a^{\prime \prime}, a^{\prime}\right) \in R$ we conclude that $a^{\prime}$ is in the right center of $R$ i.e. $\left(a^{\prime}, a^{\prime}\right) \in R$ and this case is done as witnessed by the operation generating $\left(a^{\prime}, a^{\prime}\right)$ from the generators $\left(a^{\prime}, b\right)$, $\left(b, a^{\prime}\right)$.

\section{B.3 Fundamental theorems of dichotomy proofs}

In this subsection we derive the fundamental theorems for Zhuk's and Bulatov's approach as corollaries of the results in the previous subsection.

Corollary 4.12. [The Four Types Theorem] Let $\mathbf{A}$ be an algebra, then

(a) A has a nontrivial 2-absorbing subuniverse, or

(b) A has a nontrivial center, (which is a Taylor center in the case that $\mathbf{A}$ is a Taylor algebra), or

(c) $\mathbf{A} / \alpha$ is abelian for some proper congruence $\alpha$ of $\mathbf{A}$, or

(d) $\mathbf{A} / \alpha$ is polynomially complete for some proper congruence $\alpha$ of $\mathbf{A}$.

Proof. Let us first assume that $\mathbf{A}$ is simple. If case (d) does not apply, then there exists a proper irredunant subdirect subpower of $\mathbf{A}$. By Theorem 4.7, such a relation pp-defines a strongly functional ternary relation or a proper irredundant subdirect binary relation $R$. The former situation leads to case (c) via Proposition 4.8. In the latter case, the binary relation $R \leq_{s d}$ $\mathbf{A}^{2}$ is linked (as $\mathbf{A}$ is simple) and thus it defines a proper left central relation $S \leq_{s d} \mathbf{A}^{2}$ by Proposition B.3. Now either A has a nontrivial 2-absorbing subuniverse and we are in case (a) or $S$ witnesses that we are in case (b).

In the general case, take a maximal congruence $\alpha$ of $\mathbf{A}$, apply what we have already proved to the simple algebra $\mathbf{A} / \alpha$, and observe that centers can be lifted: if $R$ is a central subuniverse of $\mathbf{A} / \alpha$, then the preimage of $R$ under the projection map $A \rightarrow A / \alpha$ is a central subuniverse of A.

Lemma B.11. The directed graph formed by the edges of any algebra is connected.

Proof. We prove the claim by induction on the the size of $\mathbf{A}$. If $\mathbf{A}$ has two elements the result follows from the classification of Boolean clones by Post [51, so we assume $|A| \geq 3$. Suppose further that $\mu_{\mathbf{A}}$ is not full, as otherwise the connectedness of $\mathbf{A}$ follows from the connectedness of proper subuniverses.

If $\mathbf{A}$ is simple, then each equivalence class of $\mu_{\mathbf{A}}$ is connected by edges by the induction hypothesis. We apply Theorem 4.7 together with Proposition 4.8, Theorem 4.10, or Theorem 4.11 to conclude that either $\mathbf{A}$ is abelian and every pair is an abelian edge, or every pair $\left(a, a^{\prime}\right) \notin \mu_{\mathbf{A}}$ 
is a majority edge, or that there exists a $\mu_{\mathbf{A}}$-block $B$ such that every pair $a \notin B, b \in B$ forms a semilattice edge $(a, b)$ (and in every case the witnessing congruence is the identity congruence).

If $\mathbf{A}$ is not simple we consider any maximal congruence $\alpha$ on $\mathbf{A}$. By the induction hypothesis the congruence blocks of $\alpha$ as well as $\mathbf{A} / \alpha$ have connected directed graphs of edges. But since any edge $(a / \alpha, b / \alpha)$ in $\mathbf{A} / \alpha$ witnessed by $\theta$ on $\operatorname{Sg}_{\mathbf{A} / \alpha}(a / \alpha, b / \alpha)$ gives rise to edge $(a, b)$ in $\mathbf{A}$ witnessed by the lifted congruence $\theta^{\prime}$ on $\operatorname{Sg}_{\mathbf{A}}(a, b)$, the proof is concluded in the non-simple case as well.

Corollary 4.13. [The Connectivity Theorem] The directed graph formed by the minimal edges of any algebra is connected.

Proof. Let A be a minimal counterexample to the theorem. By Lemma B.11 $A$ is connected by edges and it suffices to show that each two elements connected by an edge are connected by minimal edges. Let $(a, b)$ be an edge in $\mathbf{A}$ and let $\theta$ be a maximal congruence on $\operatorname{Sg}_{\mathbf{A}}(a, b)$ witnessing the edge. The blocks of $\theta$ are connected by edges (by the minimality of $\mathbf{A}$ ). Choose $a^{\prime}, b^{\prime}$ such that $\left(a, a^{\prime}\right),\left(b, b^{\prime}\right) \in \theta$ and such that $\mathbf{B}=\operatorname{Sg}_{\mathbf{A}}\left(a^{\prime}, b^{\prime}\right)$ is minimal. Note that $\operatorname{Sg}_{\mathbf{A}}(a, b) / \theta$ is isomorphic to $\mathbf{B} /\left.\theta\right|_{B}$ and thus $\left.\theta\right|_{B}$ is a maximal congruence of $\mathbf{B}$, Thus $\left(a^{\prime}, b^{\prime}\right)$ is a minimal edge of the same type as the edge $(a, b)$ and the corollary is proved.

\section{Proofs for Section 5}

This section of the appendix contains proofs of the theorems from Section 5 together with a a number of lemmas mentioned there, or needed for the proofs. The final subsection gives proofs of the claims in examples in Section 5 ,

\section{C.1 General facts}

Proposition 5.2. Every Taylor algebra has a minimal Taylor reduct.

Proof. Let $\mathbf{A}$ be a Taylor algebra and $p>|A|$ be a prime number. By Theorem 3.2 the algebra $\mathbf{A}$ has a cyclic term operation of a prime arity $p$. Consider a family of clones generated by such cyclic operations (the family is finite), and choose a term $t$ which defines a minimal (under inclusion) clone in this family. The algebra $(A ; t)$ is clearly a Taylor reduct of $\mathbf{A}$. If $(A ; t)$ had a proper Taylor reduct then, by Theorem 3.2 it would have a $p$-ary cyclic term operation. This contradicts the choice of $t$ and shows that $(A ; t)$ is a minimal Taylor algebra.

Proposition 5.3. Let $\mathbf{A}$ be a minimal Taylor algebra and $B \subseteq A$ be closed under an operation $f \in \mathrm{Clo}(\mathbf{A})$ such that $B$ together with the restriction of $f$ to $B$ forms a Taylor algebra. Then $B$ is a subuniverse of $\mathbf{A}$.

Proof. Choose a prime number $p>|A|$. The set $B$ together with the restriction of $f$ to $B$, call it $f^{\prime}$, forms a Taylor algebra. By Theorem $3.2\left(B ; f^{\prime}\right)$ has a cyclic operation of arity $p$. This operation is defined by a term in $f^{\prime}$, and we let $h$ to be the operation of $\mathbf{A}$ defined by the same term after replacing $f^{\prime}$ by $f$. The set $B$ is closed under $h$ and $h$ is a cyclic operation on $B$. Let $s$ be a cyclic composition of $h$ and a cyclic operation of $\mathbf{A}$ of arity $p$ denoted by $t$ ( $t$ exists by Theorem 3.2). The operation $s$ is cyclic and, more importantly, preserves $B$ as $t$ is idempotent and $s$ is cyclic on $B$. Since $\mathbf{A}$ is minimal Taylor, $\operatorname{Clo}(\mathbf{A})=\operatorname{Clo}(A ; s)$ and $B$ is a subuniverse of A.

Proposition 5.4. Any subalgebra, finite power, or quotient of a minimal Taylor algebra is a minimal Taylor algebra.

Proof. For finite powers the claim follows from the definition of a power. Let $\mathbf{A}$ be a minimal Taylor algebra and $\mathbf{B}$ be its subalgebra or quotient. We choose a prime number $p>|A|$ and a $p$-ary cyclic term operation $t$ of $\mathbf{A}$. Using Theorem 3.2 and Proposition 5.2 we find $s \in \mathrm{Clo}(\mathbf{A})$ such that $B$ together with the corresponding term operation $s^{\mathbf{B}}$ of $\mathbf{B}$ is minimal Taylor. Then the cyclic composition $h$ of $t$ and $s$ is a cyclic operation on $\mathbf{A}$ and the corresponding $h^{\mathbf{B}}$ coincides with 
$s^{\mathbf{B}}$. Since $\mathbf{A}$ is minimal Taylor, we have $\operatorname{Clo}(\mathbf{A})=\operatorname{Clo}(A ; h)$ and therefore $\operatorname{Clo}(\mathbf{B})=\operatorname{Clo}\left(B ; h^{\mathbf{B}}\right)=$ $\mathrm{Clo}\left(B ; s^{\mathbf{B}}\right)$, which completes the proof.

The following, additional, proposition is proved in a similar way.

Proposition C.1. Any term operation of a minimal Taylor algebra $\mathbf{A}$ can be obtained by identifying and permuting coordinates (and adding dummy coordinates) of a cyclic term operation of A.

Proof. Since $\mathbf{A}$ is minimal Taylor, $\operatorname{Clo}(\mathbf{A})=\operatorname{Clo}(A ; t)$ for any cyclic operation $t \in \operatorname{Clo}(\mathbf{A})$ (which exists by Theorem 3.2). The claim now follows by noting that the star composition of cyclic operations is a cyclic operation and that, since $t$ is idempotent, any term operation defined by a term in the symbol $t$ can be defined by star composing $t$ multiple times and then permuting and identifying coordinates.

\section{C.2 Absorption}

We begin by proving an auxiliary lemma. The lemma proves that a set is almost a projective subuniverse. The difference is, that the condition works only for tuples from $C$ which is not necessarily a subuniverse of $\mathbf{A}$.

Lemma C.2. Suppose $\mathbf{A}$ is a minimal Taylor algebra, $\emptyset \neq B \subsetneq C \subseteq A$, and $\operatorname{Sg}_{\mathbf{A}}\left(C^{n} \backslash B^{n}\right) \cap B^{n}=$ $\varnothing$ for every $n$. Then for every $f \in \mathrm{Clo}_{n}(\mathbf{A})$ and every essential coordinate $i$ of $f$ we have $f(\mathbf{a}) \notin B$ whenever $\mathbf{a} \in C^{n}$ is such that $a_{i} \in C \backslash B$.

Proof. Any cyclic term operation satisfies the required property (by using the compatibility with $\operatorname{Sg}_{\mathbf{A}}\left(C^{n} \backslash B^{n}\right)$ on cyclic permutations of $\left.\mathbf{a}\right)$ and the property is stable under identifying and permuting coordinates (and introducing dummy ones). The claim now follows from Proposition C.1.

Theorem 5.5. Let $\mathbf{A}$ be a minimal Taylor algebra and $B$ an absorbing set of $\mathbf{A}$. Then $B$ is a subuniverse of $\mathbf{A}$.

Proof. Let $f$ be a witness for $B$ absorbing $\mathbf{A}$ and assume, for a contradiction, that $B$ is not a subuniverse.

Let $\mathbf{A}^{\prime}$ be a reduct of $\mathbf{A}$ with all the operations from $\operatorname{Clo}(\mathbf{A})$ that preserve $B$. Since $\mathbf{A}^{\prime}$ is a proper reduct, it is not a Taylor algebra, and a quotient of a sublagebra of $\mathbf{A}^{\prime}$ is a two-element algebra whose every operation is a projection (since it is known [28] that powers can be dropped from Definition 3.1). Let $B_{0}$ and $B_{1}$ be the congruence blocks in this quotient, clearly every operation $t$ from $\operatorname{Clo}\left(\mathbf{A}^{\prime}\right)$ acts like a projection on $\left\{B_{0}, B_{1}\right\}$. Note that $f$ preserves $B$, therefore it has this property and we assume, without loss of generality, that $f$ acts like the first projection on $\left\{B_{0}, B_{1}\right\}$.

It follows from the previous paragraph that, for every $n$, the relation $S_{n}=\left(B_{0} \cup B_{1}\right)^{n} \backslash B_{0}^{n}$ (just like any other relation "built" over blocks $B_{0}$ and $B_{1}$ ) is compatible with every operation from $\operatorname{Clo}\left(\mathbf{A}^{\prime}\right)$ and is thus pp-definable from $\operatorname{Inv}(\mathbf{A})$ and $B$. Let $T_{n}$ be the relation defined by the same pp-definition with each conjunct $B(x)$ replaced by the void $A(x)$. Since $B$ absorbs $\mathbf{A}$ by $f$, we also know that $S_{n}$ absorbs $T_{n}$ by the same $f$ (see Lemma A.1)

Suppose that $T_{n} \cap B_{0}^{n} \neq \emptyset$ and choose $\mathbf{a} \in T_{n} \cap B_{0}^{n}$ and $\mathbf{b} \in B_{1}^{n}$. Then $f(\mathbf{a}, \mathbf{b}, \ldots, \mathbf{b})$ belongs to $B_{0}^{n}$ because $f$ acts like the first projection on $\left\{B_{0}, B_{1}\right\}$, and it also belongs to $S_{n}$ because $S_{n}$ absorbs $T_{n}$ by $f$. This contradiction shows that $T_{n} \cap B_{0}^{n}=\emptyset$ for every $n$. Note that $S_{n} \subseteq T_{n}$ and $T_{n}$ is a subpower of $\mathbf{A}$, hence $\operatorname{Sg}_{\mathbf{A}^{n}}\left(\left(B_{0} \cup B_{1}\right)^{n} \backslash B_{0}^{n}\right) \cap B_{0}^{n}=\emptyset$ for every $n$. We apply Lemma C.2 and consider the behaviour of $f$ : it preserves $B_{0} \cup B_{1}$ and has at least two essential coordinates (since it is a witness for a non-trivial absorption) and therefore cannot act like a projection on $\left\{B_{0}, B_{1}\right\}$ - this is a contradiction.

Theorem 5.7. The following are equivalent for any minimal Taylor algebra $\mathbf{A}$ and a set $B \subseteq A$.

(a) B 2-absorbs $\mathbf{A}$. 
(b) $R(x, y, z)=B(x) \vee B(y) \vee B(z)$ is a subuniverse of $\mathbf{A}^{3}$.

(c) $B$ is a projective subuniverse of $\mathbf{A}$

(d) $B$ is a strongly projective subuniverse of $\mathbf{A}$.

Proof. We begin with the implications that were discussed in the main part of the paper. The implications from (d) to (c) and from (c) to (b) hold for all algebras. The first one is trivial, and the second one follows immediately from the definitions. The implication from (b) to (a) fails in a trivial clone, but holds in Taylor algebras. To see this we assume (b) and let $t$ be a cyclic term operation of $\mathbf{A}$ of arity $2 k+1$. Define $f(x, y)=t(x, \ldots, x, y, \ldots y)$ where $x$ appears exactly $k+1$ times. It is easy to see that $f$ witnesses the 2 -absorption of $B$. Indeed, take any $a \in A, b \in B$ and let $\mathbf{c}_{1}=(a, \ldots, a, b, \ldots, b)$ with exactly $k+1$ 's. Let $\mathbf{c}_{2}$ be a cyclic shift of $\mathbf{c}_{1}$ by $k$ positions and $\mathbf{c}_{3}$ by $k+1$ positions. Clearly $\left(t\left(\mathbf{c}_{1}\right), t\left(\mathbf{c}_{2}\right), t\left(\mathbf{c}_{3}\right)\right) \in R$ and, as it is a constant tuple, we conclude that $t\left(\mathbf{c}_{1}\right)=f(a, b) \in B$. The case of $f(b, a)$ is similar and we are done with (b) implies (a).

For implication from (a) to (d) let $g$ be a binary operation witnessing the 2-absorption of $B$ into $A$ and $t \in \operatorname{Clo}(\mathbf{A})$ be a cyclic operation of arity, say $p$. Define $h\left(x_{1}, \ldots, x_{p}\right)$ as

$$
g\left(\cdots g\left(g\left(x_{1}, x_{2}\right), x_{3}\right), \ldots x_{p}\right)
$$

and note that $h\left(a_{1}, \ldots, a_{p}\right) \in B$ whenever at least one of the $a_{i}$ is in $B$. The same property holds for the cyclic composition, call it $s$, of $t$ and $h$ : Indeed, take a $p$-tuple a which, on some position, has an element from $B$. In the subterms of $s$ the operation $h$ is applied to cyclic shifts of a and every time, the result is in $B$. Then $t$ is applied to elements from $B$ and, by Theorem 5.5, the result is in $B$ as well. The operation $s$ is cyclic and therefore generates the whole clone of $\mathbf{A}$. Thus if any variable appearing in a term $f$ build from $s$ (in particular, a variable corresponding to an essential coordinate of $f^{\mathbf{A}}$ ) is evaluated to $B$, then the whole term is.

Next we provide examples showing that the reverse implications in Theorem 5.7 fail in Taylor algebras. We leave the verification of the simple examples as an exercise.

Example C.3. The algebra $(\{0,1\} ; x \vee(y \wedge z))$ is Taylor and $\{1\}$ is a projective universe which is not strongly projective. This shows that " $(c)$ implies (d)" in Theorem 5.7fails in Taylor algebras.

Let $n(x, y, z, w)$ be an operation on $\{0,1\}$ which is equal to $x \vee y \vee z \vee w$ everywhere, except that it is zero on the tuple $(1,0,0,0)$ and its cyclic shifts. Then $\mathbf{A}=(\{0,1\} ; n)$ is a Taylor algebra and the $R$ defined for $B=\{1\}$ in (b) of Theorem 5.7 is compatible with $\mathbf{A}$. On the other hand $B$ is not a projective subuniverse of $\mathbf{A}$ as witnessed by, for example, $n$ and thus (c) of Theorem 5.7 is false.

Finally, the Boolean lattice is an example of a Taylor algebra such that (a) of Theorem 5.7 holds, but (b) does not.

Proposition 5.8. Let $\mathbf{A}$ be a minimal Taylor algebra and $B \unlhd_{2} \mathbf{A}$.

1. If $C \leq \mathbf{A}$ then $B \cup C \leq \mathbf{A}$.

2. If $C$ is proper and $C \unlhd \mathbf{A}$ by $f$, then

(a) $B \cup C \unlhd \mathbf{A}$ by $f$, and

(b) $B \cap C \neq \emptyset$ and $B \cap C \unlhd \mathbf{A}$ by $f$.

3. Every minimal Taylor algebra $\mathbf{A}$ has a unique minimal 2-absorbing subalgebra B. Moreover, this algebra $\mathbf{B}$ does not have any nontrivial 2-absorbing subuniverse.

Proof. For (1) consider the result of applying an operation $f \in \operatorname{Clo}(\mathbf{A})$ to a tuple a. If $a_{i} \in C$ for all the essential coordinates $i$ of $f$, then the result is in $C$ (as $C \leq \mathbf{A}$ ), and if $a_{i} \in B$ for an essential $i$, then the result is in $B$ by item (d) in Theorem 5.7 .

The argument for (2.a) is similar. From (1) we know that $B \cup C$ is a subuniverse. For the absorption note that if tuple a has all but one entry in $B \cup C$, then $f(\mathbf{a}) \in B$ in case that $a_{i} \in B$ for 
an essential $i$, or $f(\mathbf{a}) \in C$ in the other case (as $C \unlhd \mathbf{A}$ by $f$ and the inessential coordinates can be substituted with elements of $C$ ). For (2.b) first note that $f$ has at least two essential coordinates. Then $B \cap C \unlhd \mathbf{A}$ follows again from item (d) in Theorem 5.7 and $B \cap C$ is nonempty since it contains $f(c, \ldots, c, b, c, \ldots, c)$ for any $b \in B$ and $c \in C$ (where $b$ is at an essential coordinate).

To prove (3) first observe that, by (2.b), the intersection $\mathbf{B}$ of all 2-absorbing subalgebras of $\mathbf{A}$ is 2-absorbing (and, in fact, any binary term operation of $\mathbf{A}$ whose both coordinates are essential can be taken as a witness). For the second part, note that if $\emptyset \neq C \unlhd_{2} \mathbf{B}$, then both absorptions $C \unlhd_{2} \mathbf{B} \unlhd_{2} \mathbf{A}$ can be witnesses by the same operation $f$ and then $f(f(f(x, y), x), f(f(y, x), y))$ witnesses $\mathbf{C} \unlhd_{2} \mathbf{A}$, so $C=B$.

We are moving on to a proof of Theorem 5.9 . The result is similar, in spirit, to Theorem 5.7 but the reasoning is a bit more technical. The following notion, which can be thought of as a common generalization of projectivity and absorption, will be useful.

Definition C.4. Let $f: A^{n} \rightarrow A$, let $B \subseteq A$, and let $g:\{0,1\}^{n} \rightarrow\{0,1\}$ be monotone. We write $f \sim_{B} g$ if $f(\mathbf{a}) \in B$ whenever $g(\mathbf{x})=1$ where $\mathbf{x}$ is the characteristic tuple of $\mathbf{a}$ (i.e. $x_{i}=1$ iff $\left.a_{i} \in B\right)$.

We begin with a basic property of the relation we just defined.

Proposition C.5. Fix $A$ and $B \subseteq A$. If $g \sim_{B} g^{\prime}$ are $n$-ary and $g_{1} \sim_{B} g_{1}^{\prime}, \ldots, g_{n} \sim_{B} g_{n}^{\prime}$ all $k$-ary, then $g\left(g_{1}, \ldots, g_{n}\right) \sim_{B} g^{\prime}\left(g_{1}^{\prime}, \ldots, g_{n}^{\prime}\right)$.

Proof. Let $g, g_{1}, \ldots, g_{n}$ and $g^{\prime}, g_{1}^{\prime}, \ldots, g_{n}^{\prime}$ be as in the statement of the proposition. Take any a $\in$ $A^{k}$ and let $\mathbf{x}$ be its characteristic $k$-tuple (with respect to $B$ ). Assume that $g^{\prime}\left(g_{1}^{\prime}, \ldots, g_{n}^{\prime}\right)(\mathbf{x})=1$ and note that if $g_{i}^{\prime}(\mathbf{x})=1$ then $g_{i}(\mathbf{a}) \in B$. Therefore the characteristic $n$-tuple of $\left(g_{1}(\mathbf{a}), \ldots, g_{n}(\mathbf{a})\right)$ is above the tuple $\left(g_{1}^{\prime}(\mathbf{x}), \ldots, g_{n}^{\prime}(\mathbf{x})\right)$ and since $g^{\prime}$ applied to the latter tuple outputs 1 , it outputs 1 on the former as well. But this, by the definition, implies that $g\left(g_{1}(\mathbf{a}), \ldots, g_{n}(\mathbf{a})\right) \in B$ and the proposition is proved.

Note that the previous proposition is especially useful, when the algebra $\mathbf{A}$ is generated by a single operation. More formally if $B \subseteq A$ and $g \sim_{B} g^{\prime}$, then any $f \in \operatorname{Clo}(A ; g)$ is $\sim_{B}$ related to some $f^{\prime} \in \operatorname{Clo}\left(\{0,1\} ; g^{\prime}\right)$. Indeed, for any fixed $k$, it suffices to start with $\operatorname{proj}_{i} \sim_{B} \operatorname{proj}_{i}$ (for all $i \leq k$ ) where the first projection is on $A$ and the second on $\{0,1\}$ and apply the previous proposition as many times as needed. This is in fact exactly how we prove "(a) implies (d)" in Theorem 5.9 - the proof of this implication is extracted to the following theorem.

Theorem C.6. Let $\mathbf{A}$ be a minimal Taylor algebra. There exists an arity preserving map $f \mapsto f^{\prime}$ from $\mathrm{Clo}(\mathbf{A})$ to $\mathrm{Clo}(\{0,1\} ;$ maj) such that:

- if a set $B$ 3-absorbs $A$ then $f \sim_{B} f^{\prime}$, and

- for every prime $p>|A|$ there is a cyclic term of $\mathbf{A}$ of arity $p$ which is mapped to maj $_{p}$.

Proof. For any prime $p>|A|$, we have a $p$-ary cyclic operation, say $t$, in A. If $t$ satisfies $t \sim_{B}$ maj $_{p}$ then one can generate, as in discussion after Proposition C.5, a subdirect relation $\sim_{B}$ between $\operatorname{Clo}(\mathbf{A})$ and $\operatorname{Clo}(\{0,1\} ; \mathrm{maj})$. Once this is done then, for a any $f \in \operatorname{Clo}(\mathbf{A})$, we can choose any operation $\sim_{B}$ related to $f$ and fix it to be $f^{\prime}$. Such a map satisfies the conclusion of the theorem, for the fixed $B$. If initial condition, i.e. $t \sim_{B} \operatorname{maj}_{p}$, held for a number of $B$ 's the conclusion will hold for all of them. Our induction parameter is the size of the set of $B$ 's.

Given such a set $\mathcal{B}$ (of 3 -absorbing subsets of $A$ ) and a cyclic operation $t \in \mathrm{Clo}_{p}(\mathbf{A}$ ), such that $t \sim_{B} \operatorname{maj}_{p}$ for every $B \in \mathcal{B}$, we will find another cyclic term operation $s$ which will still work for any $B \in \mathcal{B}$ and, additionally, for a new 3 -absorbing subset $C$ in $\mathbf{A}$. The claim will then follow by induction since we can start with the empty $\mathcal{B}$ and any $p$-ary cyclic term operation of $\mathbf{A}$ (in such a case we can choose an arbitrary map $f \mapsto f^{\prime}$ sending $t$ to $\operatorname{maj}_{p}$ ).

Let $f \mapsto f^{\prime}$ be a map compatible with $\sim_{B}$ for all $B \in \mathcal{B}$ and such that $t \mapsto \operatorname{maj}_{p}$. Let $f$ be a witness for $C \unlhd_{3} \mathbf{A}$, and let $f^{\prime}$ be an image of $f$. Finally, let $h$ be the $p$-ary term operation of $\mathbf{A}$ 
defined from $f$ by the same term as a term defining maj $_{p}$ from maj (the latter term exists since $\mathrm{Clo}(\{0,1\}$, maj) is the clone of monotone selfdual operations - see the discussion in Section 5) and let $s$ be the cyclic composition of $t$ and $h$. Our aim is to verify the inequality for $s \sim_{B} \operatorname{maj}_{p}$ and any $B \in \mathcal{B} \cup\{C\}$.

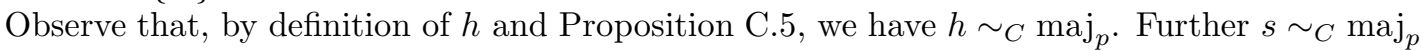
by the definition of $s$, Proposition C.5 and the fact that $B \leq \mathbf{A}$ (which follows from Theorem [5.5). We thus further concentrate on the case $B \in \mathcal{B}$. If $f^{\prime}$ (the image of $f$ from the previous paragraph) is maj, then $h \sim_{B}$ maj $_{p}$ and further $s \sim_{B}$ maj $_{p}$ for all $B \in \mathcal{B}$ by the same reasoning. And this case is done.

Otherwise $f^{\prime}$ is a projection (again by the structure of the majority clone) and Proposition C.5 implies that $h \sim_{B} \operatorname{proj}_{i}$ for some $i$. But then, again using Proposition C.5, a $k$-shift of $h$ (i.a. a term obtained by cyclic shift of arguments of $h$ by $k$ positions) is $\sim_{B}$ related to $\operatorname{proj}_{i+k} \bmod p$. Finally $s \sim_{B}$ maj $_{p}$ by the definition of $s$ (and, again, Proposition C.5). This finishes the proof of the theorem.

We now prove a version of Theorem 5.9 formulated in terms of the relation $\sim_{C}$. Note that in the formulation we reordered the items to better match the strength of the concepts.

Theorem C.7. The following are equivalent for any minimal Taylor algebra $\mathbf{A}$ and a set $B \subseteq A$.

(a) B 3-absorbs $\mathbf{A}$.

(b) $B$ is a center of $\mathbf{A}$

(c) The relation $R(x, y)=B(x) \vee B(y)$ is a subuniverse of $\mathbf{A}^{2}$.

(d) for every $f \in \operatorname{Clo}(\mathbf{A})$ there exists $g \in \operatorname{Clo}(\{0,1\}$; maj $)$ such that $f \sim_{B} g$.

Moreover, if $B=\{b\}$, then these items are equivalent to

(e) B absorbs $\mathbf{A}$.

Proof. We will begin by showing that (c) is equivalent to (d) in every algebra. For the implication from (d) to (c) take any $n$-ary operation $f$ of algebra $\mathbf{A}$, and let $g$ be such that $f \sim_{B} g$. Take two tuples a, $\mathbf{a}^{\prime}$ such that $\left(a_{1}, a_{1}^{\prime}\right), \ldots,\left(a_{n}, a_{n}^{\prime}\right) \in R$. If $f(\mathbf{a}) \in B$ then $\left(f(\mathbf{a}), f\left(\mathbf{a}^{\prime}\right)\right) \in R$ and we are done, so suppose $f(\mathbf{a}) \notin B$. Let $\mathbf{x}$ be the characteristic tuple of $\mathbf{a}$; by definition of $\sim_{B}$ we have $g(\mathbf{x})=0$. On the other hand, the definition of $R$ and the choice of $\mathbf{a}, \mathbf{a}^{\prime}$ implies that $\mathbf{x}^{\prime}$ - the characteristic tuple of $\mathbf{a}^{\prime}$ - is greater than or equal to $\mathbf{1}-\mathbf{x}=\left(1-x_{1}, \ldots, 1-x_{n}\right)$. Since $g$ is in $\operatorname{Clo}(\{0,1\} ;$ maj $)$, the clone of monotone selfdual operations, we get $g\left(\mathbf{x}^{\prime}\right) \geq g(\mathbf{1}-\mathbf{x})=g(\mathbf{1})-g(\mathbf{x})=1$, therefore $f\left(\mathbf{a}^{\prime}\right) \in B$. This proves the first implication.

For the implication from (c) to (d), note that to every $n$-ary operation $f \in \operatorname{Clo}(\mathbf{A})$ we can associate $g^{\prime}:\{0,1\}^{n} \rightarrow\{0,1\}$ by putting $g^{\prime}(\mathbf{x})=1$ if an only if $f(\mathbf{a}) \in B$ for all a with the characteristic tuple greater than or equal to $\mathbf{x}$. The operation $g^{\prime}$ is monotone by definition, and clearly $f \sim_{B} g^{\prime}$. From $g^{\prime}$ we define $g$ in the following way: for every $\mathbf{x}$ we put $g^{\prime}(\mathbf{x})=g(\mathbf{x})$ unless $g^{\prime}(\mathbf{x})=g^{\prime}(\mathbf{1}-\mathbf{x})=1$ and $x_{0}=0$ - in this case we put $g(\mathbf{x})=0$. The operation $g$ is clearly monotone, and we obviously have $f \sim_{B} g$. We will show that $g$ is self-dual, which will conclude a proof of (d). Indeed, if $g$ is not self-dual we have $g^{\prime}(\mathbf{x})=g^{\prime}(\mathbf{1}-\mathbf{x})=0$. From the definition of $g^{\prime}$ we have two tuples $\mathbf{a}$ and $\mathbf{a}^{\prime}$ with characteristic tuples greater than or equal to $\mathbf{x}$ and $\mathbf{1}-\mathbf{x}$ respectively and such that $f(\mathbf{a}), f\left(\mathbf{a}^{\prime}\right) \in A \backslash B$. Note that for every $i$ we have $R\left(a_{i}, a_{i}^{\prime}\right)$, and by compatibility $\left(f(\mathbf{a}), f\left(\mathbf{a}^{\prime}\right)\right) \in R$, but this contradicts that condition that both $f(\mathbf{a})$ and $f\left(\mathbf{a}^{\prime}\right)$ are outside $B$.

Next, we assume (d) and aim to prove (b). Again, no assumptions on the algebra is necessary. We set $\{0,1\}$ to be the universe of $\mathbf{C}$ and for every basic operation of $\mathbf{A}$, say $f^{\mathbf{A}}$, put $f^{\mathbf{C}}=g$ where $g$ is any operation such that $f \sim_{B} g$. Clearly $\operatorname{Clo}(\mathbf{C}) \subseteq \operatorname{Clo}(\{0,1\} ;$ maj $)$ and therefore $\mathbf{C}$ has no 2-absorbing subuniverses. Moreover we claim that the relation $R=A \times\{0\} \cup B \times\{1\}$ is a subuniverse of $\mathbf{A} \times \mathbf{C}$. Take $\left(a_{1}, y_{1}\right), \ldots,\left(a_{n}, y_{n}\right) \in R$, any $n$-ary symbol $f$ and let $\mathbf{x}$ be the 
characteristic tuple of $\mathbf{a}$. If $f^{\mathbf{A}}(\mathbf{a}) \in B$ we are done, so $f^{\mathbf{A}}(\mathbf{a}) \in A \backslash B$ and therefore $f^{\mathbf{C}}(\mathbf{x})=0$. But $\mathbf{y} \leq \mathbf{x}$ and so $f^{\mathbf{C}}(\mathbf{y})=0$ and the proof is done.

For (b) implies (a) see Proposition 4.3 and for (a) implies (d) see Theorem C.6. Finally (a) always implies (e). For the inverse implication we will use Proposition B.8. By assumption, $B=\{b\} \unlhd \mathbf{A}$. Take any $a \in A \backslash B$ and consider $\operatorname{Sg}_{\mathbf{A}^{2}}((a, b),(b, a))$. If $(a, a)$ is an element of this algebra, then there is a term operation $t$ satisfying $t(a, b)=a=t(b, a)$ and, by Proposition 5.3 the set $\{a, b\}$ is a subuniverse. Since $t$ acts on $\{a, b\}$ as a semilattice with absorbing element $a$, the subalgebra of $\mathbf{A}$ with universe $\{a, b\}$ (which is minimal Taylor by Proposition 5.4) is termequivalent to the semilattice with absorbing element $a$, but then $\{b\}$ can not be an absorbing subuniverse. This contradiction shows that the assumption of Proposition B.8 are satisfied and that $\{b\}$ 3-absorbs $\mathbf{A}$ as required.

Theorem 5.9 now easily follows, we just need to deal with the Taylor part in item (c).

Theorem 5.9. The following are equivalent for any minimal Taylor algebra $\mathbf{A}$ and a set $B \subseteq A$.

(a) B 3-absorbs $\mathbf{A}$.

(b) $R(x, y)=B(x) \vee B(y)$ is a subuniverse of $\mathbf{A}^{2}$.

(c) B is a (Taylor) center of $\mathbf{A}$.

(d) there exists $\mathbf{C}$ with $\operatorname{Clo}(\mathbf{C}) \subseteq \operatorname{Clo}(\{0,1\}$; maj) such that $R(x, y)=B(x) \vee(y=0)$ is a centrality witness.

Moreover, if $B=\{b\}$, then these items are equivalent to

(e) B absorbs $\mathbf{A}$.

Proof. The proof of the previous theorem shows that all the items but " $B$ is a Taylor center of A" are equivalent to the witness of centrality in item (d). If $\operatorname{Clo}(\mathbf{C})=\operatorname{Clo}(\{0,1\}$, maj), then $B$ is a Taylor center. In the other case, when $\operatorname{Clo}(\mathbf{C})$ is the clone of projections, all the term operations are $\sim_{B}$-related to a projection, so $B$ is projective and then strongly projective by Theorem 5.7 The witness for $B$ being a Taylor center can then be taken e.g. $R=(A \times\{$ rock $\}) \cup(B \times C)$ where $\mathbf{C}$ is term-equivalent to the rock-paper-scissors algebra.

Proposition 5.11 is a simple consequence of Theorem C.6.

Proposition 5.11. Let $\mathbf{A}$ be a minimal Taylor algebra and $B, C \unlhd_{3} \mathbf{A}$.

1. $B \cup C \leq \mathbf{A}$

2. If $B \cap C \neq \emptyset$ then $B \cap C \unlhd_{3} \mathbf{A}$.

3. If $B \cap C=\emptyset$ then $B^{2} \cup C^{2}$ is a congruence on the algebra with universe $B \cup C$ and the quotient is term-equivalent to a two-element majority algebra.

Proof. All the items are proved using a cyclic operation $t$ of an odd arity $p$ such that $t \sim_{B}$ maj $_{p}$ and $t \sim_{C} \mathrm{maj}_{p}$, and such an operation is provided by Theorem C.6.

Note that $t$ generates the whole clone of $\mathbf{A}$. For (1) and (3) we apply $t$ to a tuple consisting of elements from $B \cup C$. The result will be from $B$ if the majority of the arguments is from $B$ and the same for $C$. Thus (1) and (3) holds.

For $(2)$ take $f(x, y, z)=t(x, \ldots, x, y, \ldots, y, z, \ldots, z)$ where the number of $x$ 's, $y$ 's and $z$ 's is roughly equal (more precisely, the number of $x$ 's and $y$ 's is at least $p / 2$ and the same is true for the other two pairs). By construction $f$ is a witness both for $B \unlhd_{3} \mathbf{A}$ and for $C \unlhd_{3} \mathbf{A}$, and thus for $B \cap C$ as well.

Finally, we record the following fact for future reference.

Proposition C.8. Let $\mathbf{A}$ be a minimal Taylor algebra and $\mathbf{C} \unlhd_{3} \mathbf{B} \unlhd_{3} \mathbf{A}$. Then $\mathbf{C} \unlhd_{3} \mathbf{A}$. 
Proof. Since absorption is transitive (see Subsection A.3), it is enough to verify that $(a, a) \in$ $(A \backslash C)^{2}$ is not in $\operatorname{Sg}(C \times\{a\} \cup\{a\} \times C)$ and apply Proposition B.8. If $a \notin B$, this follows from the fact that the relation $B(x) \vee B(y)$ is a subuniverse of $\mathbf{A}^{2}$ (see Theorem [5.9) and if $a \in B \backslash C$, this follows from the fact that the relation $C(x) \vee C(y)$ on $B$ is a subuniverse of $\mathbf{B}^{2}$.

\section{C.3 Edges}

Theorem 5.12. Let $(a, b)$ be an edge (semilattice, majority, or abelian) of a minimal Taylor algebra $\mathbf{A}$ and $\theta$ a witnessing congruence of $\mathbf{E}=\operatorname{Sg}_{\mathbf{A}}(a, b)$.

(a) If $(a, b)$ is a semilattice edge, then $\mathbf{E} / \theta$ is term equivalent to a two-element semilattice with absorbing element $b / \theta$.

(b) If $(a, b)$ is a majority edge, then $\mathbf{E} / \theta$ is term equivalent to a two-element majority algebra.

(c) if $(a, b)$ is an abelian edge, then $\mathbf{E} / \theta$ is term equivalent to an affine Mal'cev algebra of an abelian group isomorphic to $\mathbb{Z} / p_{1}^{k_{1}} \times \cdots \times \mathbb{Z} / p_{i}^{k_{i}}$ for distinct primes $p_{1}, \ldots, p_{i}$ and positive integers $k_{1}, \ldots, k_{i}$, where $\mathbb{Z} / m$ denotes the group of integers modulo $m$.

Moreover a semillatice edge is witnessed by exactly one congruence of $\mathbf{E}$, and that congruence is maximal. The same holds for majority edges.

Proof. In (a) there is a binary term operation acting like the semilattice operation on $\{a / \theta, b / \theta\}$ with top element $b / \theta$. By Proposition 5.3 together with Proposition [5.4 the set $\{a / \theta, b / \theta\}$ is a subuniverse of $\mathbf{E} / \theta$ and thus equal to it. By the classification of Post [51] and minimality of $\mathbf{E} / \theta$ we conclude (a). The case of (b) is identical, except the operation is a ternary majority. In (c), Theorem 3.4 implies that we have the Mal'cev operation $x-y+z$ for some abelian group. Taking into account the structure of finite abelian groups and the fact that $E$ is two-generated, we get the conclusion.

For the moreover part, say we have two different congruences $\theta$ and $\gamma$ (put $\delta=\gamma \cap \theta$ ) witnessing the same semilattice (resp. majority) edge. If we have binary (resp. ternary) operation acting as a semilattice operation $t$ with matching top elements (or a majority operation) on both $\{a / \theta, b / \theta\}$ and $\{a / \gamma, b / \gamma\}$, then $t$ acts as a semilattice (majority) on $\{a / \delta, b / \delta\}$. But then, by Proposition 5.3 , $E=a / \delta \cup b / \delta$ which contradict the fact that $\theta$ and $\gamma$ were different.

It remains to obtain an operation acting as a semilattice on both semilattice edges. In the semilattice case both $b / \theta$ and $b / \gamma$ are 2-absorbing subuniverses of $\mathbf{E}$ and, by Theorem 5.7 any non-trivial binary reduct of a cyclic term is such an operation. In the majority case the reasoning is identital except that all the sets $a / \theta, b / \theta, a / \gamma, b / \gamma$ are 3 -absorbing and all these absorptions can be witnessed by a single term (e.g. a term obtained by carefully identifying variables in a cyclic term).

Proposition 5.13. Let $(a, b)$ be a minimal edge in a minimal Taylor algebra. Then $\mathbf{E}=\operatorname{Sg}_{\mathbf{A}}(a, b)$ has a unique maximal congruence equal to $\mu_{\mathbf{E}}$. In particular, minimal edges have unique types.

Proof. Let $\mathbf{E}=\operatorname{Sg}_{\mathbf{A}}(a, b)$ and let $\theta$ be a congruence on $\mathbf{E}$ witnessing the edge. Since $(a, b)$ is minimal, $\theta$ is necessarily a maximal congruence of $\mathbf{E}$. Since the only simple affine Mal'cev algebras are (up to isomorphism) the affine Mal'cev algebras of $\mathbb{Z} / p$ for a prime $p$, we have by Theorem 5.12 that $\mathbf{E} / \theta$ is term-equivalent to a two-element semilattice, a two-element majority algebra, or an affine Mal'cev algebra of a group isomorphic to $\mathbb{Z} / p$. None of these algebras have a nontrivial subalgebra and so, by minimality, there is no congruence incomparable with $\theta$. Indeed, if a congruence $\alpha$ is incomparable with maximal $\theta$, then at least one $\alpha$-block $B$ intersects two of the $\theta$-blocks. Since $B / \theta \leq \mathbf{A}$ and $\mathbf{A} / \theta$ does not have nontrivial subalgebras, then $B$ intersects all of the blocks; in particular the blocks containing $a$ and $b$. By minimality of the edge $(a, b)$, we get $B=E$, a contradiction. Similarly, since $\theta \subseteq \mu_{E}$, also $\mu_{E}=\theta$.

Proposition 5.14 is an immediate consequence of the following proposition. 
Proposition C.9. Let A be minimal Taylor algebra:

1. If $\mathbf{B} \unlhd_{2} \mathbf{A}$ and $a \in A \backslash B$ there exists $b \in B$ such that $(a, b)$ is a semilattice edge and $\operatorname{Sg}_{\mathbf{A}}(a, b)=\{a, b\}$.

2. If $\mathbf{B} \unlhd_{3} \mathbf{A}$ and $a \in A \backslash B$ there exists $b \in B$ such that:

(a) $(a, b)$ is a semilattice edge and $\operatorname{Sg}_{\mathbf{A}}(a, b)=\{a, b\}$, or

(b) $(a, b)$ is a majority edge and $\operatorname{Sg}_{\mathbf{A}}(a, b) \cap B$ is one of the two blocks of the congruence witnessing it.

Proof. We will prove both items simultaneously. First we choose $b \in B$ such that $\operatorname{Sg}_{\mathbf{A}}(a, b)$ is minimal, under inclusion in the set $\left\{\operatorname{Sg}_{\mathbf{A}}(a, b)\right\}_{b \in B}$. We let $\mathbf{C}=\operatorname{Sg}_{\mathbf{A}}(a, b)$ and $D=C \cap B$, and note that, for every $b^{\prime} \in D$, we have $C=\operatorname{Sg}_{\mathbf{A}}\left(a, b^{\prime}\right)$.

We put $R=\operatorname{Sg}_{\mathbf{C}^{2}}((a, b),(b, a))$. The first step is to show that if $D^{2} \cap R \neq \emptyset$ then $(b, b) \in R$ and $(a, b)$ is semilattice edge such that $\operatorname{Sg}_{\mathbf{C}}(a, b)=\{a, b\}$. Indeed, if $D^{2} \cap R \neq \emptyset$ then $(D+R) \cap D \neq \emptyset$ and $a \in D+R$ and consequently $D+R=C$. Further, if $D+R=C$ then there exists $b^{\prime} \in D$ such that $\left(b^{\prime}, b\right) \in R$, but then $\{b\}-R$ contains both $a$ and $b^{\prime}$ and thus $\{b\}-R=A$; in particular $(b, b) \in R$. We showed that there is a binary term operation acting on $\{a, b\}$ as a join-semilattice operation with top $b$. By Proposition [5.3, $\{a, b\}$ is a subuniverse of $\mathbf{A}$, by Proposition 5.4 the subalgebra with this subuniverse is a minimal Taylor algebra, which is clearly term equivalent to a two-element semilattice.

Going back to the claims in the proposition, if $B \unlhd_{2} \mathbf{A}$, then a pair in $D^{2} \cap R$ is produced by a single application of the operation witnessing the 2 -absorption to $(a, b)$ and $(b, a)$. This finishes the 2-absorption case.

We are left with the case of $B \unlhd_{3} \mathbf{A}$ and $R \cap D^{2}=\emptyset$. Note that, by Theorem 5.9, $R \subseteq$ $B(x) \vee B(y)$ and thus $D+R=C \backslash D$ is a ternary absorbing subuniverse of $\mathbf{C}$. By Proposition 5.11 . we conclude that the partition of $C$ into $D$ and $C \backslash D$ defines a congruence of $\mathbf{C}$ and the quotient modulo this congruence is term equivalent to a two-element majority algebra. This finishes the proof.

Proposition 5.14. Let $(a, b)$ be a minimal semilattice edge in a minimal Taylor algebra. Then $\{a, b\}$ is a subuniverse of $\mathbf{A}$, so $\operatorname{Sg}_{\mathbf{A}}(a, b)=\{a, b\}$ and the witnessing congruence is the equality.

Proof. Let $(a, b)$ be as in the statement. Put $\mathbf{A}^{\prime}=\operatorname{Sg}_{\mathbf{A}}(a, b)$ and $\theta$ a congruence witnessing the edge. The subalgebra $b / \theta 2$-absorbs $\mathbf{A}^{\prime}$, and Proposition C.9 provides $b^{\prime}$ in $b / \theta$ such that $\operatorname{Sg}_{\mathbf{A}^{\prime}}\left(a, b^{\prime}\right)=\left\{a, b^{\prime}\right\}$. Since $(a, b)$ is a minimal edge, we get $b=b^{\prime}$.

\section{C.4 Absorption and edges}

The first lemma of this subsection proves one direction in Theorems 5.18 and 5.20 .

Lemma C.10. Let $\mathbf{A}$ be a minimal Taylor algebra and $B \subseteq A$. If $B$ absorbs $\mathbf{A}$, then $B$ is stable under all the abelian and semilattice edges. Moreover, if $B$ 2-absorbs $\mathbf{A}$, then $B$ is stable under all the edges.

Proof. Assume that $B$ absorbs $\mathbf{A}$ and $(b, a)$ is an edge with witnessing congruence $\theta$ of $\mathbf{E}:=$ $\operatorname{Sg}_{\mathbf{A}}(b, a)$ such that $b / \theta$ intersects $B$. By Theorem [5.5, $B$ is a subuniverse of $\mathbf{A}$. As $B \unlhd \mathbf{A}$ we also have $B \cap E \unlhd \mathbf{E}$ and then $b / \theta \in(B \cap E) / \theta \unlhd \mathbf{E} / \theta$.

By Theorem 5.12, if $(b, a)$ is a semilattice edge, then $\mathbf{E} / \theta$ is term equivalent to the two element semilattice with absorbing element $a / \theta$; if $(b, a)$ is an abelian edge, then $\mathbf{E} / \theta$ is term equivalent to an affine Mal'cev algebra. In both cases, the description of term operations in these algebras given at the beginning of Section 5 implies $(B \cap E) / \theta=E / \theta$, so each $\theta$-block indeed intersects $B$. If, additionally, $B$ 2-absorbs $\mathbf{A}$, then $(B \cap E) / \theta \unlhd_{2} \mathbf{E} / \theta$ and we get the same conclusion by the description of term operations in the two-element majority algebra. 
Our next aim is to prove the other direction in Theorem 5.18, that subsets stable under all the edges are 2-absorbing. As we will often work with minimal semilattice edges and their sequences, the following terminology will be useful.

Definition C.11. Let A be a minimal Taylor algebra.

- An s-edge is a minimal semilattice edge.

- An s-walk is a sequence $a_{1}, \ldots, a_{k} \in A$ such that $\left(a_{i}, a_{i+1}\right)$ is an s-edge for every $i \in$ $\{1, \ldots, k-1\}$.

- $A$ set $B \subseteq A$ is s-closed if $a \in B$ whenever $(b, a)$ is an s-edge with $b \in B$.

Recall (Proposition 5.14) that for an s-edge $(a, b)$ in a minimal Taylor algebra A, the set $\{a, b\}$ as a subuniverse of $\mathbf{A}$ (so the witnessing congruence for this edge is the equality on $\{a, b\}$ ). It follows that every set stable under semilattice edges is s-closed.

Before proving Theorem 5.18 we give a useful criterion for s-closed subsets to be binary absorbing.

Proposition C.12. Suppose that B is an s-closed subset of a minimal Taylor algebra A. Then the following are equivalent:

(a) B 2-absorbs $\mathbf{A}$,

(b) for all $a \in A \backslash B$ and all $b \in B$, the algebra $\operatorname{Sg}_{\mathbf{A}}(a, b)$ has a proper 2-absorbing subuniverse,

(c) for all $a \in A$ and all $b \in B$, there is a directed $s$-walk from a to an element in $B$ which is contained in $\operatorname{Sg}(a, b)$.

Proof. To see that (a) implies (b), note that $B \unlhd_{2} \mathbf{A}$ implies that $B \cap \operatorname{Sg}_{\mathbf{A}}(a, b) \unlhd_{2} \operatorname{Sg}_{\mathbf{A}}(a, b)$.

We prove that (b) implies (c) by induction on the size of $\mathbf{E}=\operatorname{Sg}_{\mathbf{A}}(a, b)$. Let $C$ be a nontrivial 2-absorbing subuniverse of $\operatorname{Sg}_{\mathbf{A}}(a, b)$. By Proposition C.9 applied twice, there exists an s-edge $\left(b, b^{\prime}\right)$ and $\left(a, a^{\prime}\right)$ with $b^{\prime}, a^{\prime} \in C$ and, since $B$ is s-closed, we have $b^{\prime} \in B$. Now $B \cap \operatorname{Sg}\left(a^{\prime}, b^{\prime}\right)$ is an s-closed subset of $\mathbf{E}^{\prime}=\operatorname{Sg}\left(a^{\prime}, b^{\prime}\right)$ which is a proper subalgebra of $\mathbf{E}$ (since $E^{\prime} \subseteq C \subsetneq E$ ) so either $a, a^{\prime}$ is already an s-walk into $B$ or we can append this walk by an s-walk from $a^{\prime}, b^{\prime}$ (in $E^{\prime}$ ) obtained from induction hypothesis, and we are done.

Now suppose that (c) holds. Let $\mathbf{F}$ be the subalgebra of $\mathbf{A}^{A^{2}}$ formed by all the binary term operations of $\mathbf{A}$ (recall Subsection $\mathbf{A . 2}$ ) and let $\mathbf{R} \leq \mathbf{A}^{X}$ be the projection of $\mathbf{F}$ onto the set of coordinates $X=\{(a, b),(b, a): a \in A, b \in B\}$. Notice that any $f \in R \cap B^{X}$ witnesses that $B$ is a 2-absorbing subset of $\mathbf{A}$, therefore it is enough to show that $R$ intersects $B^{X}$. We assume the converse.

Let $Y \subseteq X, n \in \mathbb{N}$ be such that

- $Y$ is maximal such that the projection of $R$ onto $Y$ intersects $B^{Y}$ (note that $Y \subsetneq X$ by the assumption), and

- $n$ is the smallest number such that there exists a coordinate $\mathbf{x} \in X \backslash Y$, there exists $f \in R$ with $\left.f\right|_{Y} \in B^{Y}$, and there exists an s-walk $a_{1}, \ldots, a_{n}$ in $\operatorname{proj}_{\mathbf{x}} R$ with $a_{1}=f(\mathbf{x})$ and $a_{n} \in B$ (and fix such a pair $\mathbf{x}$ and an s-walk).

Note that the definition in the second item makes sense since $\operatorname{proj}_{\mathbf{x}} R$ contains an element of $B$ (as witnessed by one of the two projection operations in $R$ ), so such an s-walk $a_{1}, \ldots, a_{n}$ exists by (c). Now $\left\{a_{2}\right\}$ is a 2-absorbing subuniverse of $\operatorname{Sg}_{\mathbf{A}}\left(a_{1}, a_{2}\right)=\left\{a_{1}, a_{2}\right\}$ since $\left(a_{1}, a_{2}\right)$ is an s-edge. Therefore the subniverse $R^{\prime}$ of $\mathbf{R}$ obtained by fixing the coordinate $\mathbf{x}$ of $R$ to $\left\{a_{2}\right\} 2$-absorbs the subalgebra of $\mathbf{R}$ obtained by fixing the same coordinate to $\left\{a_{1}, a_{2}\right\}$. By Proposition C.9. there exists an s-edge from $f$ to $R^{\prime}$, giving us an s-edge $(f, g)$ in $R$ for some $g \in R$ with $g(\mathbf{x})=a_{2}$. Since $(f, g)$ is an s-edge, the pair $(f(\mathbf{y}), g(\mathbf{y}))$ is, for any $\mathbf{y} \in Y$, also an s-edge (witnessed by the same term). Recalling that $B$ is s-closed we obtain $\left.g\right|_{Y} \in B^{Y}$. But $g(\mathbf{x})$ starts the s-walk $a_{2}, \ldots, a_{n}$, which is one shorter than the original walk $a_{1}, \ldots, a_{n}$, a contradiction to the minimality of $n$ (in case of $n>2$ ) or maximality of $Y$ (in case of $n=2$ ). 
The last lemma before proving Theorem 5.20 provides some information about two-generated algebras that are not necessarily coming from edges. It is a weaker version of Theorem C.18.

Lemma C.13. If $\mathbf{A}$ is a minimal Taylor algebra which is generated by two distinct elements $a, b \in A$, then either $\mathbf{A}$ has a nontrivial abelian quotient, or $\mathbf{A}$ has a nontrivial 3-absorbing subuniverse.

Proof. Factoring by a maximal congruence we can assume that $\mathbf{A}$ is simple (as 3-absorbing subuniverses lift from quotients). If $\mathbf{A}$ has a ternary strongly functional subpower or a binary irredundant subdirect subpower, then we are done by Proposition 4.8 or Corollary 4.5 (since the subpower is then necessarily linked by simplicity of $\mathbf{A})$. Also, if $(a, b)$ is an s-edge, we are done as well.

Otherwise, $\operatorname{Sg}_{\mathbf{A}^{2}}((a, b),(b, a))$ is not full (it does not contain $(b, b)$ ), so it must be a graph of a bijection - an automorphism of $\mathbf{A}$. Moreover, by Theorem 4.7, $\mathbf{A}$ has no proper irredundant subdirect subpowers, therefore $\operatorname{Sg}_{\mathbf{A}^{3}}((b, a, a),(a, b, a),(a, a, b))$ is full. In particular, it contains $(a, a, a)$, therefore there exists a term operation $t$ such $t(b, a, a)=t(a, b, a)=t(a, a, b)=a$. Since A has an automorphism switching $a$ and $b$ we get that $t$ acts as a majority operation on $\{a, b\}$, so $\{a\} 3$-absorbs $\{a, b\}$, which is equal to $A$ by Theorem 5.3 , and we have found a non-trivial 3 -absorbing subuniverse.

Theorem 5.18. The following are equivalent for any minimal Taylor algebra $\mathbf{A}$ and a set $B \subseteq A$.

(a) B 2-absorbs A.

(b) $B$ is stable under all the edges.

Proof. Lemma C.10 shows that (a) implies (b), and so we concentrate on the other direction.

By Proposition C.12, it is enough to show that for any $a \in A \backslash B$ and any $b \in B$, there is an s-edge from $a$ to an element of $\operatorname{Sg}(a, b) \cap B$. We may assume without loss of generality that $\operatorname{Sg}(a, b)=A$, and we will inductively assume that the theorem is true for algebras of size smaller than $|A|$.

If $\mathbf{A}$ has a nontrivial affine quotient, then since $B$ is stable under abelian edges, $B$ must intersect the congruence class which contains $a$, so we can apply the inductive assumption to find an s-edge from $a$ to $B$ which is contained in that congruence class. Otherwise, Lemma C.13 implies that there is some nontrivial ternary absorbing subalgebra $\mathbf{C}$ of $\mathbf{A}$.

Next we show that $C$ intersect $B$. Suppose the contrary and apply Proposition C.9 to the element $b$ and $C \unlhd_{3} \mathbf{A}$ - we get an s-edge from $b$ from $C$, which is impossible since $B$ is s-closed, or a majority edge $(b, c)$ with $c \in C$ such that the witnessing congruence $\theta$ of $\mathbf{E}=\operatorname{Sg}_{\mathbf{A}}(b, c)$ has two blocks one of which is $E \cap C$. By the induction hypothesis $B \cap E 2$-absorbs $\mathbf{E}$, but then $B / \theta=b / \theta$ 2-absorbs $\mathbf{E} / \theta$, which is impossible for a majority edge in a minimal Taylor algebra (recall Theorem 5.12 and the description of the majority clone).

Now we have $C \cap B \neq \emptyset$ and we can apply the induction hypothesis to see that $C \cap B 2$ absorbs $\mathbf{C}$. This implies that $C \cap B$ is a ternary absorbing subalgebra of $\mathbf{A}$ by the transitivity of 3-absorption stated in Proposition C.8. Thus we may assume without loss of generality that $C \subseteq B$.

By PropositionC.9, there is either a semilattice edge from $a$ to $C$, or there is an element $c \in C$ such that $\operatorname{Sg}(a, c)$ has a congruence $\theta$ for which $\operatorname{Sg}(a, c) / \theta$ is a two-element majority algebra with congruence classes $\operatorname{Sg}(a, c) \cap C$ and $\operatorname{Sg}(a, c) \backslash C$. In the first case we are done, so suppose we are in the second case. Since $\operatorname{Sg}(a, c)$ must contain some edge from one congruence class of $\theta$ to the other, and since $C \subseteq B$ and $B$ is stable under majority edges, we see that $B$ has nonempty intersection with the congruence class $\operatorname{Sg}(a, c) \backslash C$. Then we may apply the induction hypothesis to $\operatorname{Sg}(a, c) \backslash C$ to see that there is a semilattice edge from $a$ to $(\operatorname{Sg}(a, c) \backslash C) \cap B$, which finishes the proof.

Notice that the proof of (b) implies (a) shows a stronger claim: it is enough to assume that $B$ is stable under abelian edges, it is s-closed, and that there is no (minimal) majority edge $(b, a)$, witnessed by a congruence $\theta$ on $\operatorname{Sg}(b, a)$, such that $b / \theta \subseteq B$ and $a / \theta \cap B=\emptyset$. 
Our next project is to prove that (b) implies (a) in Theorem 5.20, The following lemma combines the relational description of strongly projective subuniverses in Proposition $\mathrm{A.3}$ and the sufficient condition for abelianess in Proposition 4.8.

Lemma C.14. Let $\mathbf{A}$ be a simple algebra and $R \leq_{s d} \mathbf{A}^{3}$ be a symmetric ternary relation whose projection to each pair of coordinates is full. For any $a \in A$ denote by $R_{a}$ the relation $R_{a}(x, y) \equiv$ $R(a, x, y)$. Then either $\mathbf{A}$ is abelian or the set $B=\left\{a \in A: R_{a}\right.$ is linked $\}$ is a nonempty strongly projective subuniverse of $\mathbf{A}$.

Proof. Consider the ternary relation $S$ defined by $S(x, y, z)$ if $y$ and $z$ are linked in $R_{x}$. Note that $S$ is pp-definable from $R$ so $S$ is a subuniverse of $\mathbf{A}^{3}$. Since the projection of $R$ onto each pair of coordinates is full, the relation $R_{a}$ is subdirect for every $a$. If $R_{a}$ is linked, then $S_{a}$ (defined analogously to $R_{a}$ ) is full. If $R_{a}$ is not linked, then it is a graph of a bijection $A \rightarrow A$ (since $\mathbf{A}$ is simple), so $S_{a}$ is the identity relation. In summary, $S$ is equal to the relation $B(x) \vee(y=z)$. By Proposition A.3, $B$ is strongly projective.

If $B$ is empty, then every $R_{a}$ is a graph of a bijection. But then the original relation $R$ is strongly functional, so $\mathbf{A}$ is abelian by Proposition 4.8 , finishing the proof.

We are ready to prove the main tool for Theorem 5.20. It will be useful to use the following concept. A subuniverse $R$ of $\mathbf{A}^{X}$ is $B$-essential if $R$ does not intersect $B^{X}$ but every projection of $R$ onto all but one of the coordinates intersects a power of $B$. By Proposition A.2 $\{a\}$ absorbs A by a term operation of arity $n$ if and only if it does not have any $\{a\}$-essential subpower of arity $n$.

Theorem C.15. Let $\mathbf{A}$ be a Taylor algebra and $a \in A$. Suppose that $\{a\}$ does not absorb $\mathbf{A}$ but does absorb every proper subalgebra of $\mathbf{A}$ that contains $\{a\}$. Then

- A has a nontrivial abelian quotient or

- there exists a nonempty projective subuniverse $B$ of $\mathbf{A}$ such that $a \notin B$.

Proof. First, observe that it is enough to prove the claim for simple A. Indeed, if $\alpha$ is a maximal congruence of $\mathbf{A}$, then either $a / \alpha$ absorbs $A$ (in which case $\{a\}$ absorbs $\mathbf{A}$ by transitivity of absorption, a contradiction to the assumptions), or it does not (in which case we apply the simple case - observe that projective subuniverses lift from quotients). Assume therefore that $\mathbf{A}$ is simple.

We further assume that every relation $S \leq_{s d} \mathbf{A}^{2}$ whose left center contains $a$ is full. Indeed, if it is not, then by Corollary B.6 either $\mathbf{A}$ has a proper projective and 2-absorbing subuniverse $B$ or the left center of $R$ is an absorbing subuniverse of $\mathbf{A}$. By transitivity of absorption and the assumptions, no absorbing subuniverse of $\mathbf{A}$ can contain $a$. Therefore $B$ would be a projective subuniverse not containing $a$ and the proof would be concluded.

Next we observe that there exists a symmetric $\{a\}$-essential relation $R \leq \mathbf{A}^{\mathbb{N}}$. Indeed, for any arity $n$ there exists by Proposition A.2 some $\{a\}$-essential relation, i.e., containing tuples $\left(b_{1}, a, a, \ldots\right),\left(a, b_{2}, a \ldots, a\right), \ldots$ and not containing $(a, a, \ldots, a)$. Taking an element $b=b(n)$ that appears most times among the $b_{i}$ and fixing (and projecting out) the other coordinates to $a$ we get an $\{a\}$-essential relation of arity at least $n /|A|$ containing $(b, a, a \ldots),(a, b, a, \ldots), \ldots$ Finally, by taking $R=\operatorname{Sg}_{\mathbf{A}^{\mathbb{N}}}((b, a, a, \ldots),,(a, b, a, \ldots), \ldots)$, where $b$ is an element that appears infinitely many times among the $b(n)$, we get a symmetric $\{a\}$-essential relation.

For a symmetric relation $R \leq \mathbf{A}^{\mathbb{N}}$, denote $B(R)=\{b \in A:(b, a, a, \ldots) \in R\}$, and take a symmetric $\{a\}$-essential relation $R$ (i.e., $a \notin B(R) \neq \emptyset$ ) such that $B(R)$ is maximal. Set $B=B(R)$. We will show that $R$ either pp-defines a ternary relation forcing strong projectivity or abelianess, or $R$ is a witness for $B$ being a projective subuniverse, i.e., after fixing any co-finite collection coordinates to $a, R$ becomes $B\left(x_{1}\right) \vee B\left(x_{2}\right) \vee \cdots \vee B\left(x_{k}\right)$ (see Proposition 3.8). Since $a \notin B$ we will be done.

We claim that $\operatorname{Sg}_{\mathbf{A}}(a, b)=A$ for every $b \in B$. Indeed, otherwise $R$ defined by $\operatorname{Sg}_{\mathbf{A}^{\mathbb{N}}}\{(b, a, a, \ldots)$, $(a, b, a, \ldots), \ldots\}$ is an $\{a\}$-essential symmetric subuniverse of $\left(\operatorname{Sg}_{\mathbf{A}}(a, b)\right)^{\mathbb{N}}$, so $\{a\}$ does not absorb $\mathrm{Sg}_{\mathbf{A}}(a, b)$ by Proposition $\mathrm{A.2}$, contradicting the assumptions of the theorem. 
Next observe that the projection of $R$, call it $Q$, to any two distinct coordinates is full, even after fixing all but one of the remaining coordinates to $a$. Indeed, such a projection $Q$ contains the pairs $(a, a),(a, b),(b, a)$ (for any $b \in B)$. As $\operatorname{Sg}(a, b)=A$, the left center of $Q$ contains $\{a\}$ so it is full by the assumption made in the second paragraph of the proof.

Denote $S$ the projection of $R$ onto two of the coordinates after fixing the rest to $a$, i.e., $S(x, y) \equiv R(x, y, a, a, a, \ldots)$. Note that if we fix a coordinate of $R$ to a set $C$ and project onto the remaining coordinates, then we get a symmetric relation $R^{\prime}$ with $B\left(R^{\prime}\right)=C+S$, which will be $\{a\}$-essential iff $a \notin C+S \neq \emptyset$.

Consider the relation $R^{\prime}$ obtained by fixing a coordinate of $R$ to $B+S$ and projecting to the remaining coordinates. Observe that $R^{\prime}$ is symmetric and the set $B^{\prime}:=B\left(R^{\prime}\right)=B+S+S$ contains $B$ (note that $S$ is symmetric) therefore, by the maximality of $B=B(R)$, either $B^{\prime}=B$ or $a \in B^{\prime}$.

If $B^{\prime}(=B+S+S)=B$, then $S$ is not linked, so it is a graph of a bijection by simplicity of $\mathbf{A}$. We fix all but arbitrarily selected three coordinates of $R$ to $a$, project onto the three coordinates, and call $T$ the obtained subuniverse of $\mathbf{A}^{3}$. The relation $T$ has full projection to the first two coordinates (as we argued above) and the binary relation obtained by fixing a coordinate to $a$ is a graph of a bijection. LemmaC.14now implies the either $\mathbf{A}$ is abelian or $\mathbf{A}$ has a nonempty strongly projective subuniverse that does not contain $a$. Since strong projectivity implies projectivity, we are done.

If $a \in B^{\prime}$, then there exists $\left(b, b^{\prime}\right) \in(B \times B) \cap S$. By similar reasoning to the proof of Proposition C.9. $S$ contains $B\left(x_{1}\right) \vee B\left(x_{2}\right)$ (it contains $\left(a, b^{\prime}\right)$ as well as $\left(b, b^{\prime}\right)$ so also $A \times\left\{b^{\prime}\right\}$ since $\operatorname{Sg}\{a, b\}=A$; then for any $b^{\prime \prime} \in B$ it contains $\left(b^{\prime \prime}, b^{\prime}\right)$ and $\left(b^{\prime \prime}, a\right)$ so $\left\{b^{\prime \prime}\right\} \times A$ as well). Furthermore, $S$ is equal to $B\left(x_{1}\right) \vee B\left(x_{2}\right)$ since otherwise there is $\left(c, c^{\prime}\right) \in S, c, c^{\prime} \notin B$, but then we fix a coordinate to $c$. The obtained $R^{\prime}$ has $B\left(R^{\prime}\right)=\{c\}+S$, which contains $B$ and $c^{\prime}$ but does not contain $a$, a contradiction to the maximality of $B=B(R)$.

Pick any $b \in B$ and consider $T(x, y)=R(x, y, b, a, a, \ldots)$. It contains $(a, b),(b, a),(a, a)$, therefore it is the whole $A^{2}$ (as the left center of $T$ contains $a$ ). It follows that after fixing all but three coordinates to $a$, the ternary relation contains $B\left(x_{1}\right) \vee B\left(x_{2}\right) \vee B\left(x_{3}\right)$. Similarly as above, it cannot contain any other triple, such as $\left(c, c^{\prime}, c^{\prime \prime}\right)$, because we would fix two coordinates to $\left(c, c^{\prime}\right)$ and get a relation $R^{\prime}$ with larger $B\left(R^{\prime}\right)$ (containing $B$ and $\left.c^{\prime \prime}\right)$.

If the algebra $\mathbf{A}$ is minimal Taylor we can stop the proof here, because we have already obtained the subpower $B\left(x_{1}\right) \vee B\left(x_{2}\right) \vee B\left(x_{3}\right)$ (see Theorem 5.7). For general Taylor algebras, we can by induction obtain the subpower $B\left(x_{1}\right) \vee B\left(x_{2}\right) \vee \cdots \vee B\left(x_{n}\right)$ for every $n$, in a completely analogous way.

Theorem 5.20 is a simple consequence of the results proved so far.

Theorem 5.20. Any absorbing set of a minimal Taylor algebra $\mathbf{A}$ is stable under semilattice and abelian edges. Moreover, for any $b \in A$ the following are equivalent.

(a) $\{b\}$ absorbs $\mathbf{A}$

(b) $\{b\}$ is stable under semilattice and abelian edges.

Proof. That absorbing sets are stable under semilattice and abelian edges and that (a) implies (b) follows from Lemma C.10. That (b) implies (a) follows from Theorem C.15. Indeed, if $\{b\}$ does not absorb $\mathbf{A}$ then we take a minimal subalgebra $\mathbf{B}$ of $\mathbf{A}$ such that $\{b\}$ does not absorb $\mathbf{B}$ and apply the theorem. Either $\mathbf{B}$ has a nontrivial abelian quotient, in which case $\{b\}$ is not stable under abelian edges, or $\mathbf{B}$ has a nonempty projective subuniverse $C$ that does not contain $b$. In the latter case there exists a minimal semilattice edge $(b, c)$ with $c \in C$ (by Proposition C.9) and so $\{b\}$ is not s-closed; in particular, it is not stable under semilattice edges.

The "unified operations" theorem, Theorem 5.22 , is a simple consequence of the results proved so far as well. The following proposition implies a refined version discussed in Subsection 5.4

Proposition C.16. Let $t$ be an $n$-ary cyclic term operation of a minimal Taylor algebra $\mathbf{A}$ and $B \unlhd_{3} \mathbf{A}$. Then for any $m \geq n / 2$ and any $\mathbf{a} \in A^{n}$ such that $a_{1}, \ldots, a_{m} \in B$ we have $t(\mathbf{a}) \in B$. 
Proof. Let $\mathbf{b}$ be the tuple obtained by cyclically shifting the tuple a by $n-m$ positions. Since $m \geq n / 2$, each pair $\left(a_{i}, b_{i}\right)$ is in the relation $B\left(x_{1}\right) \vee B\left(x_{2}\right)$; and since $t$ is cyclic, we have $t(\mathbf{a})=t(\mathbf{b})$. But $t$ is compatible with $B\left(x_{1}\right) \vee B\left(x_{2}\right)$ by Theorem [5.9, therefore $t(\mathbf{a}) \in B$.

Theorem 5.22. Every minimal Taylor algebra A has a ternary term operation $f$ such that if $(a, b)$ is an edge witnessed by $\theta$ on $\mathbf{E}=\operatorname{Sg}_{\mathbf{A}}(a, b)$, then

- if $(a, b)$ is a semilattice edge, then $f(x, y, z)=x \vee y \vee z$ on $\mathbf{E} / \theta$ (where $b / \theta$ is the top);

- if $(a, b)$ is a majority edge, then $f$ is the majority operation on $\mathbf{E} / \theta$ (which has two elements);

- if $(a, b)$ is an abelian edge, then $f(x, y, z)=x-y+z$ on $\mathbf{E} / \theta$;

- $f$ witnesses all the ternary absorptions $B \unlhd_{3} \mathbf{A}$;

- any binary operation obtained from $f$ by identifying two arguments witnesses all the binary absorptions $B \unlhd_{2} \mathbf{A}$.

Proof. Choose positive integers $n, k, l$ such that $n=1(\bmod |A| !), k=1(\bmod |A| !), 2 k+l=n$, and $2 k \geq l$. Let $m=k$. Note that $\mathbf{A}$ has a cyclic operation $t$ of arity $n$ : every prime divisor $p$ of $n$ is greater than $|A|$ and thus there exists a cyclic term operation $t_{p}$ of arity $p$ by Theorem 3.2 The operation $t$ can then be obtained by a star composition of the $t_{p} \mathrm{~s}$.

Define $f$ by

$$
f(x, y, z)=t(\underbrace{x, x, \ldots, x}_{k \times}, \underbrace{y, y, \ldots, y}_{l \times}, \underbrace{z, z, \ldots, z}_{m \times}) .
$$

Because $\mathbf{E} / \theta$ and $\mathbf{A}$ are both minimal Taylor algebras, the operation $f$ satisfies the second and the fourth item by using the cyclicity of $t$ and Proposition C.16, and it satisfies the first and the fifth item by Theorem 5.7. Note that for these claims we actually only need $k+l, k+m, l+m \geq n / 2$.

For the third item note that a cyclic operation in an affine Mal'cev algebra is equal to $\sum_{i=1}^{n} a x_{i}$ $(\bmod q)$ where $n a=1(\bmod q)$. By simple arithmetic we conclude that if the conditions on $k, l, m$ hold, then the ternary operation $f$ is $x-y+z$.

Now our aim is to prove a refined version of Lemma C.13 with the final goal of Theorem 5.23 . The following lemma will be used to produce a proper 3-absorbing subuniverse containing a generator.

Lemma C.17. Let $\mathbf{A}$ be a minimal Taylor algebra generated by $a, b \in A$. Suppose that $C \unlhd_{3} \mathbf{A} i s$ nontrivial and 2-absorbs a subalgebra of $\mathbf{A}$ containing a and a subalgebra of $\mathbf{A}$ contaning $b$. Then A has a proper 3-absorbing subuniverse containing a or $b$.

Proof. For simplicity, we will say that a set 2-absorbs $a$ (or $b$ ) if it absorbs a subalgebra containing $a$ (or $b)$. Let $S=\operatorname{Sg}_{\mathbf{A}^{2}}((a, b),(b, a))$. As a first step we obtain a nontrivial 3-absorbing subuniverse $D$ that 2 -absorbs $a$ and $b$ and such that $(c, d) \in S \cap(C \times D)$ : take $D=C$ if $C+S=A$, or $D=C+S$ in the other case. Let $f$ be a witness for $(c, d) \in S$, that is, $f(a, b)=c$ and $f(b, a)=d$. Next observe that if $a$ is in $C$ or $D$, then the goal is reached. Suppose henceforth that $C \cup D$ does not contain $a$.

By Theorem C.6. $f \sim_{C} f^{\prime}$ and $f \sim_{D} f^{\prime}$ for some monotone selfdual binary operation, i.e., $f^{\prime}$ is one of the two projections. Suppose $f^{\prime}$ is the first projection, so $f(C, A) \subseteq C$ and $f(D, A) \subseteq D$. From these inclusions, from $f(a, b), f(b, a) \in C \cup D$, and from $f(\{a, b\}, C) \subseteq C, f(\{a, b\}, D) \subseteq D$ (by strong projectivity from Theorem [5.7) it follows that $C \cup D \cup\{b\}$ absorbs the set $E=$ $C \cup D \cup\{a, b\}$ by $f$. It is therefore enough to show that $E$ is a subuniverse of $\mathbf{A}-$ then $E=\operatorname{Sg}(a, b)$ and the proof will be concluded.

Let $t$ be a cyclic term operation of arity $p$, take

$$
s\left(x_{1}, \ldots, x_{p}\right)=f\left(f\left(f\left(\ldots f\left(x_{1}, x_{2}\right), x_{3}\right), x_{4}\right) \ldots x_{p}\right)
$$

and let $h$ be the cyclic composition of $t$ and $s$. The result of applying $s$ to a tuple $\mathbf{e} \in E^{p}$ is, by the properties above, in $C \cup D$ whenever e is not the constant tuple of $a$ 's or $b$ 's. For any other tuple, 
we have $h(\mathbf{e}) \in t(C \cup D, \ldots, C \cup D)$, which is by Item 1) of Proposition 5.11 a subset of $C \cup D$. Since $h$ is a cyclic operation and it preserves $E$, then $E$ is a subuniverse of $\mathbf{A}$ by Proposition 5.3 and the proof is concluded.

Theorem C.18. If $\mathbf{A}$ is a minimal Taylor algebra which is generated by two distinct elements $a, b \in A$, then either $\mathbf{A}$ has a nontrivial abelian quotient, or at least one of $a, b$ is contained in a proper 3-absorbing subuniverse of $\mathbf{A}$.

Proof. Just like in the proof of Lemma C.13 we can assume that A is simple and non-abelian, and assume for contradiction that neither of the generators $a, b$ is contained in a proper absorbing 3 -subuniverse. We also assume that $\mathbf{A}$ has no nontrivial 2-absorbing subuniverse, as otherwise Lemma C.17 gives a contradiction immediately.

First we will prove that every reflexive relation $S \leq_{s d} \mathbf{A}^{2}$ is either the equality or the full relation. Suppose not. Then, by simplicity of $\mathbf{A}, S$ is linked. By replacing $S$ by $S-S$, perhaps multiple times if necessary, we can further assume that $S-S=A^{2}$ while $S$ is still not full. Then there is some $c \in A$ such that $(a, c),(b, c) \in S$, and since $A=\operatorname{Sg}(a, b)$, we see that $S$ has a nontrivial right center $C$ and is still reflexive.

We claim that $C$ 2-absorbs the subalgebra $\mathbf{D}$ of $\mathbf{A}$ with universe $\{a\}+S$ (that contains $a$ since $S$ is reflexive). By PropositionC.12, we just need to check that for any $d \in D \backslash C$ and any $c \in C$, $\mathrm{Sg}(d, c)$ has a proper binary absorbing subuniverse. To see this, note that the binary relation $T=S \cap(A \times \operatorname{Sg}(d, c))$ is a subdirect subuniverse of $\mathbf{A} \times \operatorname{Sg}(d, c)$ (since $c$ is in the right center of $S$ ) and $a$ is contained in the left center of $T$ ( since $d, c \in\{a\}+S$ ). Since $d \notin C$, we see that $T$ is a proper subset of $A \times \operatorname{Sg}(d, c)$, so the left center of $T$ is a proper subalgebra of $\mathbf{A}$. Thus if $\operatorname{Sg}(d, c)$ has no proper binary absorbing subuniverse, then the left center of $T$ is a center of $\mathbf{A}$, and $a$ is contained in a proper ternary absorbing subuniverse of $\mathbf{A}$ by Proposition 4.3, a contradiction. The same argument shows that $C$ 2-absorbs a sublagebra containing $b$. Application of Lemma C.17 then gives us a contradiction, so we have proved that every reflexive subdirect subuniverse of $\mathbf{A}^{2}$ is the equality or the full relation.

Observe next that $S=\operatorname{Sg}_{\mathbf{A}^{2}}((a, b),(b, a)$ is a graph of a bijection. Indeed, otherwise we can use the same argument as above with the little tweak that $D$ may contain $b$ (not $a$ ) since $S$ is not reflexive (but contains $(a, b))$.

Finally, consider the subdirect symmetric ternary relation $R=\operatorname{Sg}_{\mathbf{A}^{3}}\{(a, a, b),(a, b, a),(b, a, a)\}$. Since the projection $\operatorname{proj}_{12} R$ of $R$ onto the first two coordinates contains $(a, a),(a, b)$ and $\operatorname{Sg}(a, b)=$ $A$, then $\operatorname{proj}_{12} R$ has a left center that contains $a$. The center cannot be proper since otherwise we get a nontrivial 2-absorbing subuniverse or the center of $\operatorname{proj}_{12} R$ is a Taylor center of $\mathbf{A}$ and we apply Corollary B.5 to get a contradiction. Therefore $\operatorname{proj}_{12} R=A^{2}$. If $(a, a, a) \in R$, then the same argument as in Lemma C.13 gives us that $(a, b)$ is a majority edge, a contradiction. The binary relation $T(x, y) \equiv R(a, x, y)$ is thus subdirect (as the projection onto any two coordinates is full) but not full (as $(a, a) \notin T$ ). Observe that $T+S$ is reflexive but cannot be full (as otherwise $T=(T+S)+S$ is full as well), therefore it is the equality relation and hence $T$ is a graph of a bijection. It follows that the strongly projective subuniverse $B$ from LemmaC.14 is neither empty (as $\mathbf{A}$ is not abelian) nor equal to $A$ (as $a \notin B$ ). Theorem 5.7 gives us that $B$ 2-absorbs $\mathbf{A}$, a contradiction.

While we do not have an example of a minimal Taylor algebra in which some pair (or its reverse) is not an edge, we do not have a strong support for the non-existence of such an example either. However, we conjecture the following.

Conjecture C.19. If A is a minimal Taylor algebra which is generated by two elements a, $b \in$ A such that neither $(a, b)$ nor $(b, a)$ is an edge, then there are proper 3-absorbing subuniverses $C, D \unlhd_{3}$ A such that $a \in C$ and $b \in D$.

Theorem 5.23 follows from the following more general theorem.

Theorem C.20. Let $\mathbf{A}$ be a minimal Taylor algebra and $f_{1}, \ldots, f_{n}$ any collection of term operations of $\mathbf{A}$ such that at least one $f_{i}$ has a nontrivial restriction to every $\operatorname{Sg}_{\mathbf{A}}(a, b) / \theta$ where $(a, b)$ is an edge and $\theta$ is a maximal congruence of $\operatorname{Sg}_{\mathbf{A}}(a, b)$. Then $f_{1}, \ldots, f_{n}$ generate the clone of $\mathbf{A}$. 
Proof. Let $\mathbf{G}$ be the reduct of $\mathbf{A}$ with basic operations $f_{1}, \ldots, f_{n}$. It is known (see e.g. [28]) that powers can be dropped from Definition 3.1 so we just need to show that for any $a, b \in G$, there is no two-element quotient $\mathrm{Sg}_{\mathbf{G}}(a, b) / \alpha$ such that each $f_{i}$ acts as a projection. Suppose for contradiction that there was such a pair $a, b$ and congruence $\alpha$ on $\operatorname{Sg}_{\mathbf{G}}(a, b)$, and choose such $a, b$ such that $\operatorname{Sg}_{\mathbf{G}}(a, b)$ is minimal. Note that $a / \alpha$ might not be a subuniverse of $\mathbf{A}$.

By Theorem C.18, we see that either $\operatorname{Sg}_{\mathbf{A}}(a, b)$ has a nontrivial abelian quotient and then $\operatorname{Sg}_{\mathbf{A}}\{a, b\} / \theta$ can be chosen of prime order by taking a maximal $\theta$ (by Theorem 5.12 ), or one of $a, b$ is contained in a proper ternary absorbing subuniverse of $\operatorname{Sg}_{\mathbf{A}}(a, b)$.

If there is a congruence $\theta$ such that $\operatorname{Sg}_{\mathbf{A}}(a, b) / \theta$ is affine of prime order, then by assumption some $f_{i}$ acts nontrivially on $\operatorname{Sg}_{\mathbf{A}}(a, b) / \theta$, so there is some ternary term operation $p \in \operatorname{Clo}_{3}\left(A ; f_{i}\right)$ such that the restriction of $p$ to $\operatorname{Sg}_{\mathbf{A}}(a, b) / \theta$ is Mal'cev (by the description of term operations of the affine Mal'cev algebra of $\mathbb{Z} / p)$. Since $f_{i}$ acts as a projection on $\operatorname{Sg}_{\mathbf{G}}(a, b) / \alpha, p$ also acts like a projection on $\operatorname{Sg}_{\mathbf{G}}(a, b) / \alpha$ - suppose without loss of generality that $p$ does not act like third projection on $\operatorname{Sg}_{\mathrm{G}}(a, b) / \alpha$. Then we have

$$
p(a, a, b) \in a / \alpha \cap b / \theta .
$$

But then $\operatorname{Sg}_{\mathbf{G}}(p(a, a, b), b) / \alpha=\operatorname{Sg}_{\mathbf{G}}(a, b) / \alpha$ and

$$
\operatorname{Sg}_{\mathbf{G}}(p(a, a, b), b) \subseteq \operatorname{Sg}_{\mathbf{G}}(a, b) \cap b / \theta,
$$

contradicting the minimality of $\operatorname{Sg}_{\mathrm{G}}(a, b)$.

Now suppose that $a$ is contained in a proper 3-absorbing subalgebra $\mathbf{C}$ of $\operatorname{Sg}_{\mathbf{A}}(a, b)$. If $C$ is 2-absorbing, then there is some s-edge going into $C$ (by Proposition C.9), and any binary term operation of $\mathbf{G}$ which acts nontrivially on this semilattice edge witnesses the 2-absorption $C \unlhd_{2} \operatorname{Sg}_{\mathbf{A}}(a, b)$ (since $C$ is strongly projective by Theorem [5.7 and both coordinates are necessarily essential). If $C$ is not a 2-absorbing subuniverse of $\operatorname{Sg}_{\mathbf{A}}(a, b)$, then $C$ is not stable under majority edges (by Lemma C.10 and Theorem (5.18), and any ternary term operation of $\mathbf{G}$ which acts nontrivially on the two-element majority quotient corresponding to this edge witnesses the ternary absorption $C \unlhd_{3} \operatorname{Sg}_{\mathbf{A}}(a, b)$. Indeed, this ternary operation is $\sim_{C}$-related to some ternary member of $g \in \operatorname{Clo}(\{0,1\}$, maj) by Theorem [5.9] but $g$ cannot be a projection (because of the majority edge) so it must be the majority operation.

Either way, we see that there is some ternary term operation $t$ in the clone generated by the $f_{i}$ which witnesses the ternary absorption $C \unlhd_{3} \operatorname{Sg}_{\mathbf{A}}(a, b)$, and we may suppose without loss of generality that $t$ acts like first projection on $\operatorname{Sg}_{\mathbf{G}}(a, b) / \alpha$. Then we have

$$
t(b, a, a) \in b / \alpha \cap C .
$$

But then $\operatorname{Sg}_{\mathbf{G}}(a, t(b, a, a)) / \alpha=\operatorname{Sg}_{\mathbf{G}}(a, b) / \alpha$ and

$$
\operatorname{Sg}_{\mathbf{G}}(a, t(b, a, a)) \subseteq \operatorname{Sg}_{\mathbf{G}}(a, b) \cap C,
$$

contradicting the minimality of $\operatorname{Sg}_{\mathbf{G}}(a, b)$ again, and concluding the proof.

Theorem 5.23. If $\mathbf{A}$ is a minimal Taylor algebra, then $\operatorname{Clo}(A ; f)=\operatorname{Clo}(\mathbf{A})$ for any operation $f$ satisfying the first three items in Theorem 5.22.

Proof. It is a consequence of Theorem C.20,

\section{C.5 Examples from Section 5}

Example 5.10. Consider the algebra $\mathbf{A}=(\{0,1,2\}, m)$ where $m$ is the majority operation such that $m(a, b, c)=a$ whenever $|\{a, b, c\}|=3$. This algebra is minimal Taylor and the set $C=\{0,1\}$ is an absorbing subuniverse of $\mathbf{A}$. However, $C$ is not a center of $\mathbf{A}$. 
Proof. The algebra is minimal Taylor because $m$ generates a minimal clone (see [32]). The set $C=\{0,1\}$ is an absorbing subuniverse of $\mathbf{A}$ as witnessed by the 4-ary operation

$$
m\left(m\left(m\left(x_{1}, x_{2}, x_{3}\right), x_{2}, x_{4}\right), x_{3}, x_{4}\right) .
$$

The set $C$ is not a center of $\mathbf{A}$ since for any potentially witnessing relation $R \leq_{s d} \mathbf{A} \times \mathbf{B}$ the subuniverse $D=2+R \leq \mathbf{B}$ satisfies $m(D, C, C) \subseteq D$ (as $m(2,1,0)=2)$ and $m(C, D, D) \subseteq D$ (as $m(0,2,2)=2)$, so $m(x, y, y)$ witnesses that $D$ is a 2-absorbing subuniverse of $\mathbf{B}$.

Example 5.15. Let $A=\{0,1,2,3\}$ and $\alpha$ the equivalence relation on $A$ with blocks $\{0,2\}$ and $\{1,3\}$. Define a symmetric ternary operation $g$ on $A$ as follows. When two of the inputs to $g$ are equal, $g$ is given by $g(a, a, a+1)=a, g(a, a, a+2)=g(a, a, a+3)=a+2$ (all modulo 4 ) and when all three inputs to $g$ are distinct, $g$ is given by $g(a, b, c)=d-1(\bmod 4)$ where $a, b, c, d$ are any permutation of $0,1,2,3$. Then $\mathbf{A}=(A ; g)$ is a minimal Taylor algebra, $\alpha$ is a congruence on $\mathbf{A}$, and each of pair of elements in different $\alpha$-blocks is a minimal majority edge where the witnessing congruence is equal to $\alpha$.

Proof. That $\mathbf{A}$ is Taylor follows from the fact that $g$ is symmetric. To show that $\mathbf{A}$ is minimal Taylor, we will show that every Taylor reduct $\mathbf{A}^{\prime}$ of $\mathbf{A}$ has a ternary cyclic term $f$, and that every ternary cyclic term $f$ of $\mathbf{A}$ generates the same clone as $g$.

Consider any Taylor reduct $\mathbf{A}^{\prime}$ of $\mathbf{A}$, and note that the congruence $\alpha$ is also a congruence on $\mathbf{A}^{\prime}$. Each congruence class of $\alpha$ is a Taylor reduct of an abelian algebra of size 2, so $\mathbf{A}^{\prime}$ has a ternary term $p$ which acts as the minority operation on the congruence classes of $\alpha$, and $\mathbf{A}^{\prime} / \alpha$ is a Taylor reduct of a majority algebra, so $\mathbf{A}^{\prime}$ has a ternary term $m$ which acts as the majority operation on $\mathbf{A}^{\prime} / \alpha$. Cyclically composing these terms, we get a ternary cyclic term $f(x, y, z)=p(m(x, y, z), m(y, z, x), m(z, x, y))$. Thus every Taylor reduct of $\mathbf{A}$ has a ternary cyclic term $f$.

To constrain the set of possible ternary cyclic terms $f$ of $\mathbf{A}$, we will use the fact that every relation of $\mathbf{A}$ which is preserved by $g$ must also be preserved by $f$. To this end, we need to find interesting relations on $\mathbf{A}$.

Note that the cyclic permutation $\left(\begin{array}{llll}0 & 1 & 2 & 3\end{array}\right)$ is an automorphism of $\mathbf{A}$, so the same is true for any reduct of $\mathbf{A}$ (equivalently, the graph of the permutation $\left(\begin{array}{llll}0 & 1 & 2 & 3\end{array}\right)$ is a binary relation on $\mathbf{A}$ which is preserved by $g$ ). One can easily verify that the binary relation $\mathbf{S}=\operatorname{Sg}_{\mathbf{A}^{2}}\{(0,1),(1,0)\}$ is equal to $\{(x, y) \mid x=y \pm 1(\bmod 4)\}$, and that there is a congruence $\theta$ on $\mathbf{S}$ with congruence classes $\{(x, y) \mid x=y+1(\bmod 4)\}$ and $\{(x, y) \mid x=y-1(\bmod 4)\}$. Additionally, the quotient $\mathbf{S} / \theta$ is isomorphic to the 2-element minority algebra.

By the above, the operation $f$ must be compatible with the automorphism ( $\left.\begin{array}{llll}0 & 1 & 2 & 3\end{array}\right)$, the binary relation $\mathbf{S}$, and the congruences $\alpha$ and $\theta$. Additionally, $f$ must act as the minority operation on $\{0,2\}$ and on $\mathbf{S} / \theta$, and $f$ must act as the majority operation on $\mathbf{A}^{\prime} / \alpha$.

The above constraints on $f$ allow us to determine every value of $f$ in terms of the value of $f(0,0,1)$. To see this, note that since $f$ acts as the minority operation on $\mathbf{S} / \theta$, we can determine the value of $f(a \pm 1, b \pm 1, c \pm 1)$ from the value of $f(a, b, c)$. For instance, we have $f(1,1,0)=f(0,0,1)-1$ $(\bmod 4)$ and $f(0,2,1)=f(1,1,0)-1(\bmod 4)=f(0,0,1)-2(\bmod 4)$. This, together with the fact that $f$ is cyclic, allows us to determine the value of $f(a, b, c)$ for every triple $a, b, c$ not all of the same parity from the value of $f(0,0,1)$. If $a, b, c$ all have the same parity, then $f$ acts as the minority operation on $\{a, b, c\}$. Since $f$ acts as majority on $\mathbf{A}^{\prime} / \alpha$, we have $f(0,0,1) \in\{0,2\}$, so there are only two possibilities for $f$. If $f(0,0,1)=0$, then $f$ is equal to $g$. Otherwise, if $f(0,0,1)=2$, then we have $g(x, y, z)=f(f(x, x, f(x, y, z)), f(y, y, f(x, y, z)), f(z, z, f(x, y, z)))$, so $f$ and $g$ generate the same clone.

Example 5.16. Let $\mathbf{A}=(\{a, b, c, d\}, p)$, where $p$ is a Mal'cev operation with the following properties. The operation $p$ commutes with the permutations $\sigma=(a c)$ and $\tau=(b d)$. The polynomials $+_{a}=p(\cdot, a, \cdot),+_{b}=p(\cdot, b, \cdot)$ define abelian groups: 


\begin{tabular}{c|ccccc|cccc}
$+{ }_{a}$ & $a$ & $b$ & $c$ & $d$ & $+{ }_{b}$ & $a$ & $b$ & $c$ & $d$ \\
\hline$a$ & $a$ & $b$ & $c$ & $d$ & $a$ & $b$ & $a$ & $d$ & $c$ \\
$b$ & $b$ & $c$ & $d$ & $a$ & $b$ & $a$ & $b$ & $c$ & $d$ \\
$c$ & $c$ & $d$ & $a$ & $b$ & $c$ & $d$ & $c$ & $b$ & $a$ \\
$d$ & $d$ & $a$ & $b$ & $c$ & $d$ & $c$ & $d$ & $a$ & $b$
\end{tabular}

Then $\mathbf{A}$ is a minimal Taylor algebra, with a unique maximal congruence $\theta$ whose congruence classes are $\{a, c\}$ and $\{b, d\}$. Each pair of elements of $\mathbf{A}$ in different congruence classes of $\theta$ is a minimal abelian edge of $\mathbf{A}$ such the witnessing congruence is $\theta$.

Proof. The algebra $\mathbf{S}=\operatorname{Sg}_{\mathbf{A}^{2}}\{(a, b),(b, a)\}$ has a congruence $\psi$ such that $\mathbf{S} / \psi$ is isomorphic to $(\mathbb{Z} / 4, x-y+z)$. Explicitly, the congruence classes of $\psi$ are $\{(a, b),(c, d)\},\{(b, a),(d, c)\}$, $\{(a, d),(c, b)\},\{(b, c),(d, a)\}$, and in this order they correspond to the elements $0,1,2,3$ of $\mathbb{Z} / 4$. Abusing notation, we will identify the congruence classes of $\psi$ with the elements of $\mathbb{Z} / 4$ in the remainder of the proof.

To prove that this example is minimal Taylor, first note that every pair of elements forms an abelian edge, so the same must be true in any Taylor reduct. Thus by Theorem 6.5] any Taylor reduct $\mathbf{A}^{\prime}$ of $\mathbf{A}$ must have a Mal'cev term $q(x, y, z)$. The restriction of $q$ to any two-element affine subalgebra or quotient must be the minority operation. This fixes the restriction of $q$ to the sets $\{a, c\}$ and $\{b, d\}$, as well as the restriction of $q$ to the quotient $\mathbf{A} / \theta$, and the fact that $\mathbb{Z} / 4$ has only one Mal'cev term forces $q$ to act on $\mathbf{S} / \psi=\mathbb{Z} / 4$ as $x-y+z$.

Similarly to the previous example, the above constraints on the term $q$ imply that $q$ is completely determined by the restriction of $q$ to the set $\{a, b\}$. For instance, we have $(q(a, b, d)$, $q(b, a, a)) / \psi=q(0,1,3)=2=(a, d) / \psi$, so from $q(b, a, a)=b$ we can conclude that $q(a, b, d)=c$. Since $q$ is Mal'cev, the only undetermined values of $q$ with all inputs from $\{a, b\}$ are the values of $q(a, b, a)$ and $q(b, a, b)$, which determine each other. Thus $q$ is completely determined by the value of $q(a, b, a)$, which is either $b$ or $d$. If $q(a, b, a)=b$, then $q$ is equal to $p$. Otherwise, if $q(a, b, a)=d$, then we have $p(x, y, z)=q(x, q(x, q(x, z, y), q(y, z, y)), z)$, so $p$ and $q$ generate the same clone.

Example 5.19. We consider the four-element algebra $\mathbf{A}=(\{0,1,2, *\}, \cdot)$ with binary operation . given by

\begin{tabular}{l|llll}
$\cdot$ & 0 & 1 & 2 & $*$ \\
\hline 0 & 0 & 2 & 1 & $*$ \\
1 & 2 & 1 & 0 & 2 \\
2 & 1 & 0 & 2 & 1 \\
$*$ & $*$ & 2 & 1 & $*$
\end{tabular}

Then A is a minimal Taylor algebra, with a semilattice edge $(0, *)$, with $\{0,1,2\}$ an affine subalgebra, and with a congruence $\theta$ corresponding to the partition $\{0, *\},\{1\},\{2\}$ such that $\mathbf{A} / \theta$ is affine. The set $\{*\}$ is stable under semilattice and majority edges and there is no minimal abelian edge $(*, a)$ with $a \neq *$. But $\{*\}$ is not an absorbing subalgebra of $\mathbf{A}$.

Proof. First we will show that every Taylor reduct of $\mathbf{A}$ is term-equivalent to $\mathbf{A}$. Since $\mathbf{A}$ has no majority edges and no $\mathbb{Z} / 2$ edges, and since every pair of elements forms an edge, the same must be true in any Taylor reduct, so any Taylor reduct must have a binary commutative term $f$ by Theorem 6.4.

Since $\mathbb{Z} / 3$ has only one idempotent binary commutative term operation, the restriction of $f$ to $\{0,1,2\}$ is given by $f(x, y)=2 x+2 y(\bmod 3)$. Similarly, the restriction of $f$ to $\{0, *\}$ is given by $f(0, *)=f(*, 0)=*$. Finally, because the values of $f$ on $\mathbf{A} / \theta$ are known, we have $f(1, *) / \theta=f(1,0) / \theta=2 / \theta$, so $f(1, *)=2$, and similarly $f(2, *)=1$. Thus $f$ is the same as the operation - displayed above.

To see that $\{*\}$ is not absorbing, note that by Theorem 5.9] and Theorem 6.3, if $\{*\}$ was absorbing, then $\{*\}$ would be 2-absorbing, and then by Theorem [5.7 the binary operation * would witness the absorption. However, we have $1 \cdot *=2 \notin\{*\}$, so $\{*\}$ is not 2-absorbing. 


\section{Proofs for Section 6: Omitting types}

Theorem 6.1. The following are equivalent for any algebra $\mathbf{A}$.

(i) A is a-free.

(ii) No subalgebra of $\mathbf{A}$ falls into case (c) in Corollary 4.12, i.e., no subalgebra of $\mathbf{A}$ has a nontrivial affine quotient.

(iii) A has a wnu term operation of every arity $n \geq 3$.

(iv) A has bounded width.

Proof. Combining the results of [4, 23] and [18, A has bounded width if and only if no subalgebra of $\mathbf{A}$ has a nontrivial abelian quotient. The proof of Theorem 2.8 in [46] shows that this is also equivalent to having wnu term operations of every arity $n \geq 3$. Thus, (i), (iii), and (iv) are equivalent. To show that (ii) implies (i), it suffices to observe that if $\mathbf{A}$ contains an abelian edge $(a, b)$ and congruence $\theta$ witnesses that, then the algebra $\operatorname{Sg}_{\mathbf{A}}(a, b) / \theta$ is abelian. Finally, to show that (i) implies (ii), assume that there is a subalgebra $\mathbf{B}$ of $\mathbf{A}$ and its congruence $\theta$ such that $\mathbf{B} / \theta$ is abelian. Then for any $a, b$ from different blocks of $\theta$, the pair $(a, b)$ is an abelian edge.

Theorem 6.2. The following are equivalent for any minimal Taylor algebra A.

(i) $\mathbf{A}$ is s-free.

(ii) Case (a) in Corollary 4.12 does not hold, that is, no subalgebra of A has a nontrivial 2absorbing subuniverse.

(iii) A has a 3-edge term operation.

(iv) A has few subpowers.

Proof. First, to show that (i) implies (ii) let $B \subseteq \mathbf{A}$ be a 2-absorbing subuniverse. If $B$ is a proper subset of $A$, then Proposition C.9 gives us a minimal semilattice edge, a contradiction.

By Theorem 2.12. of [11] the existence of a 3-edge term operation is equivalent to a so-called 3-cube operation and then (ii) implies (iii) follows from Theorem 4.5 in [43] (see also [45]) and the fact that $\mathrm{Clo}(\mathbf{A})$ is generated by a single ternary operation by Theorem 5.23 and that projective subuniverses are 2-absorbing (Proposition B.2).

From [1] it follows that (iii) implies (iv) - it is proved there that the existence of an edge (or cube) term operation is equivalent to having few subpowers.

Finally, we show that (iv) implies (i). If $\mathbf{A}$ contains a semilattice edge, then by Theorem 5.12 (a) it also has a subalgebra term equivalent to a 2-element semilattice. It is known from [11, 40, that a semilattice does not have few subpowers.

Theorem 6.3. The following are equivalent for any minimal Taylor algebra $\mathbf{A}$.

(i) $\mathbf{A}$ is m-free.

(ii) Every center (3-absorbing subuniverse of) $\mathbf{B} \leq \mathbf{A}$ 2-absorbs $\mathbf{B}$, i.e., (b) implies (a) in Corollary 4.12 in all the subalgebras of $\mathbf{A}$.

(ii') Every subalgebra of A has a unique minimal 3-absorbing subuniverse

Proof. To see that (i) implies (ii) recall that 3 -absorbing subuniverses and centers are the same by Theorem 5.9, that 3 -absorbing subuniverses are stable under abelian and semilattice edges by Theorem 5.20, and that 2-absorbing subuniverses are exactly those stable under all the edges by Theorem 5.18, By Proposition 5.8(3) (ii) implies (ii'). The fact that every majority edge defines two disjoint 3-absorbing subuniverses shows that (iii) implies (i).

Before proving Theorem 6.3 , we first verify one of the implications. 
Proposition D.1. If $\mathbf{A}$ is a minimal Taylor algebra without majority edges and $\mathbb{Z} / 2$-edges, then A has a commutative binary term operation.

Proof. By Lemma 4.4 in [3] it is enough to show that for any $a, b \in \mathbf{A}$ there is a term operation $f$ such that $f(a, b)=f(b, a)$, or in other words that the algebra $\operatorname{Sg}_{\mathbf{A}^{2}}((a, b),(b, a))$ contains a tuple of the form $(c, c)$. Suppose for contradiction that $a, b$ are chosen such that $S=\operatorname{Sg}\{(a, b),(b, a)\}$ is minimal among binary symmetric relations which do not intersect the diagonal. We may assume without loss of generality that $\operatorname{Sg}(a, b)=\mathbf{A}$. If $\mathbf{A}$ has a proper congruence $\theta$, then by induction on $|\mathbf{A}|$ we see that there is some $c \in \mathbf{A}$ such that $S \cap(c / \theta)^{2} \neq \emptyset$, but then $S \cap(c / \theta)^{2}$ is a smaller binary symmetric relation that also avoids the diagonal, a contradiction.

Suppose first that $\mathbf{A}$ has a proper 3-absorbing subuniverse $B$. If $B \cap(B+S) \neq \emptyset$, then for any $d \in B \cap(B+S)$ we have $(c, d) \in S \cap B^{2}$, so since $(c, d)$ generate a proper subalgebra of $\mathbf{A}$ we see by induction that $\operatorname{Sg}((c, d),(d, c)) \leq \mathbf{S}$ contains a diagonal element.

On the other hand, if $B \cap(B+S)=\emptyset$, then by Proposition $5.11 E=B \cup(B+S)$ must be a subuniverse of $\mathbf{A}$ with a congruence $\theta$ corresponding to the partition $B, B+S$, such that $\mathbf{E} / \theta$ is a two element majority algebra. Any pair in different blocks is then a majority edge, a contradiction.

Since A has no 3-absorbing subuniverse and is simple, it follows from Proposition C.13 that $\mathbf{A}$ is abelian (so A is term equivalent to an affine Mal'cev algebra of a group isomorphic to $\mathbb{Z} / p$ ) and from Corollary 4.5 that $S$ is a graph of a bijection $A \rightarrow A$. As $p \neq 2$ is odd by the absence of $\mathbb{Z} / 2$-edges, $S$ contains a diagonal element.

Theorem 6.4. The following are equivalent for any minimal Taylor algebra $\mathbf{A}$.

(i) $\mathbf{A}$ is $\mathrm{m}$-free and has no $\mathbb{Z} / 2$-edges.

(iii) A has a binary commutative term operation

(iii') $\operatorname{Clo}(\mathbf{A})$ can be generated by a collection of binary operations.

Proof. By Proposition D.1 (i) implies (iii), and (iii) implies (iii') by minimality of the Taylor algebra.

That (iii') implies (i) follows from the fact that the two element majority algebra and the two element affine Mal'cev algebra both have no nontrivial binary operations.

Theorem 6.5. The following are equivalent for any minimal Taylor algebra $\mathbf{A}$.

(i) $\mathbf{A}$ is sm-free.

(ii) No subalgebra of $\mathbf{A}$ has a nontrivial absorbing subuniverse.

(iii) A has a Mal'cev term operation.

Proof. To prove that (i) implies (ii) note that minimal edges have unique types (Proposition 5.13) so the algebra does not have any minimal semilattice or majority edges and then clearly no semilattice or majority edges. By Theorem 5.20, every absorbing set is stable under abelian and semilattice edges. Since A has only abelian edges, such a set has to be the whole universe of the algebra by Corollary 4.13 and the fact that no minimal (sic!) abelian edge can leave such a stable set.

Condition (ii) is the property $H A F$ from [7]. By Theorem 1.4 from the same paper $\mathbf{A}$ has a Mal'cev term operation, so (iii) holds.

Finally, if $\mathbf{A}$ has a semilattice or a majority edge $(a, b)$ witnessed by a congruence $\theta$, then the algebra $\operatorname{Sg}_{\mathbf{A}}(a, b) / \theta$ has a Mal'cev term operation, which is impossible by Proposition 5.13. Therefore (iii) implies (i)

Theorem 6.6. The following are equivalent for any minimal Taylor algebra $\mathbf{A}$.

(i) $\mathbf{A}$ is as-free. 
(iii) A has a near unanimity term operation.

(iii') A has a majority term operation.

Proof. Let us show that (i) implies (iii'). Since A has no abelian and semilattice edges, by Theorem 5.20 every 1-element subset of $\mathbf{A}$ is an absorbing subuniverse. By Theorem 5.9 every such subset is also 3 -absorbing. Finally, by Theorem 5.22 there exists a ternary operation $f$ witnessing all 3 -absorptions. Since every singleton is a 3 -absorbing subuniverse, $f$ is a majority operation.

Clearly (iii') implies (iii).

Since both a two-element semilattice and an affine Mal'cev algebra do not have a near unanimity term operation, by Theorem 5.12 (iii) implies (i). 